GEM TN-92-206

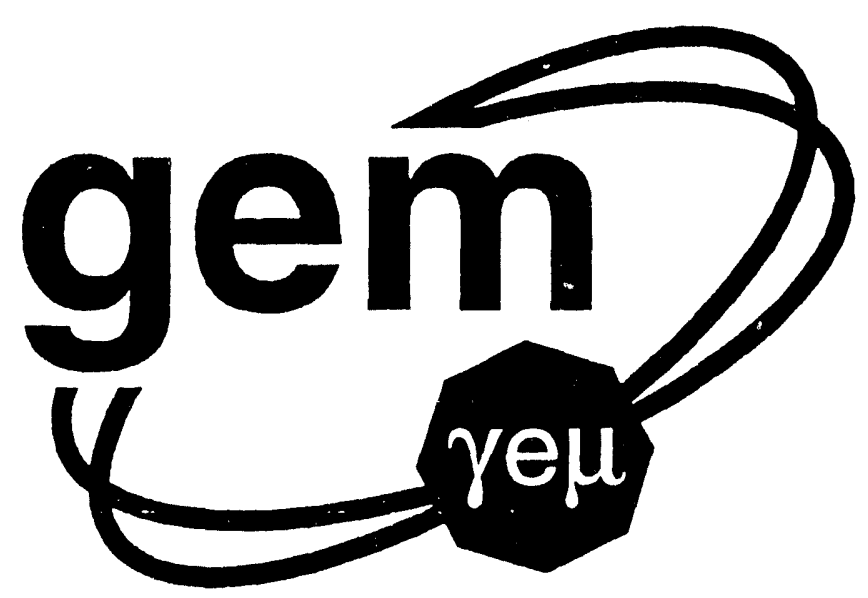

\title{
Resistive Plate Chamber Technology Review
}

\author{
M. Widgoff - Brown University \\ E. D. Alyea - Indiana University
}

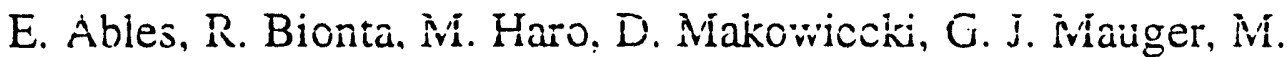
McKernan, K. Milller, P. Kamsey, C. R. Wuest Lawrence Livernore National Laboratory

Y. H. Chang, D. Chen, E. S. Hafen. P. Haridas, M. Lee. I. A. Pless,

S. Yunus - Massachusetts 'nstitute of Technology

R. Santonico - University of kume \& Instituto Nazionale di Fisica Nucleare S. Berridge, W. Bugg, P. Y. C. Du - University of Tennessee

October 6, 1992

Abstract:

A review of the resistive plate chamber technology option for the GEM muon system.

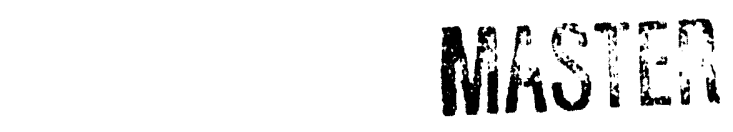



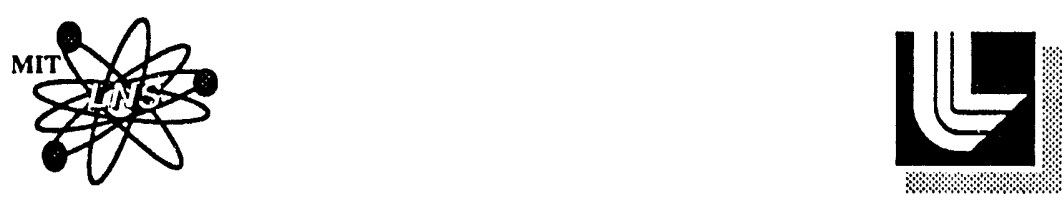

\title{
RESISTIVE PLATE CHAMBER TECHNOLOGY REVIEW
}

October 6, 1992

\author{
M. Widgoff \\ Brown Lniversity \\ E. D. Alyea \\ Indiana University
}

E. Ables, R. Bionta, M. Haro, D. Makowiecki, G. J. Mauger, M. McKernan,

K. Miller, P. Ramsey, C. R. Wuest*

Lawrence Livermore National Laboratory

Y. H. Chang, D. Chen, E. S. Hafen, P. Haridas, M. Lee, I. A. Pless*, S. Yunus Massachusetts Institute of Technology

R. Santonico

University of Rome and Instituto Nazionale di Fisica Nucleare

S. Berridge, W. Bugg, P. Y. C. Du

University of Tennessee

*Co-spokesmen 


\section{Table of Contents}

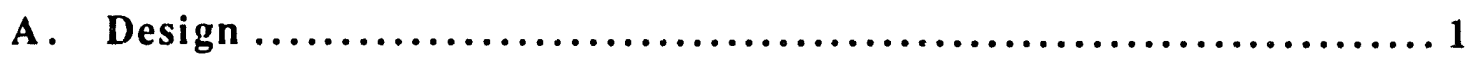

1. General Design Concept........................................................

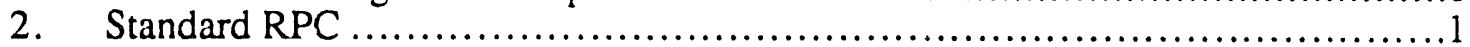

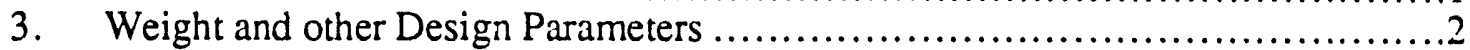

4. Wire or Strip Support............................................................

5. RPC Gas System..............................................................

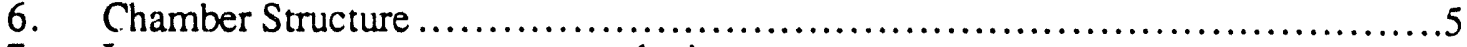

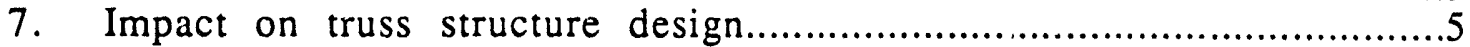

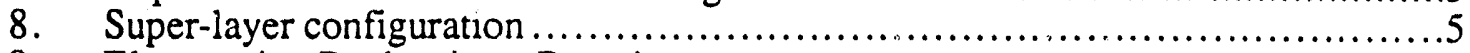

9. Electronic Packaging Requirements................................................6

10. Size Limitation...............................................................

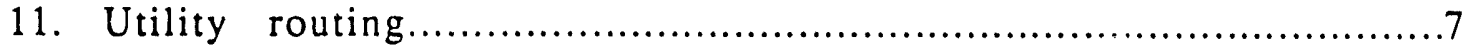

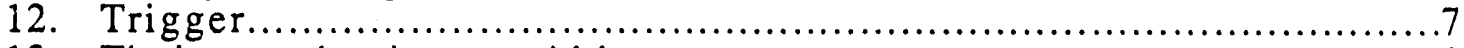

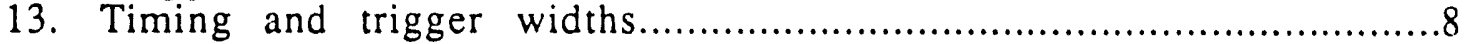

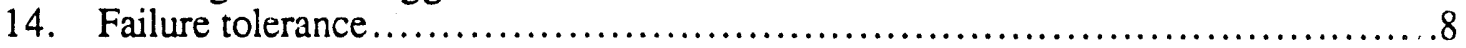

B . RPC R\&D Results $\ldots \ldots \ldots \ldots \ldots \ldots \ldots \ldots \ldots \ldots \ldots \ldots \ldots \ldots \ldots \ldots$

1. Standard Italian and Glass RPC Performance .................................9

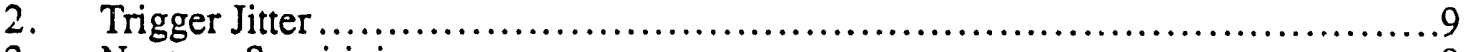

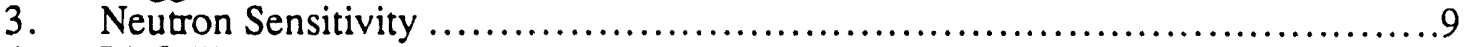

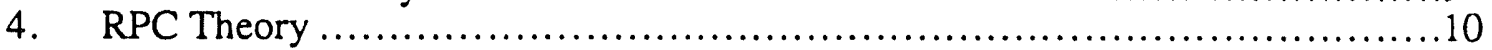

5. Alternative RPC. Materials - High Rate RPCs ...................................... 10

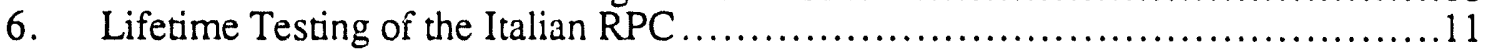

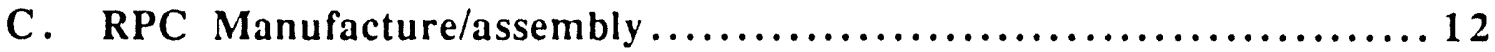

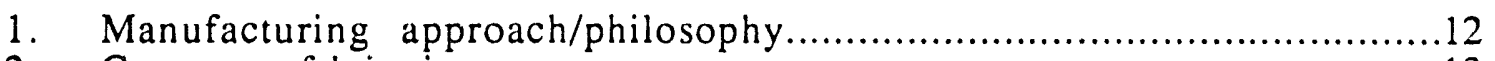

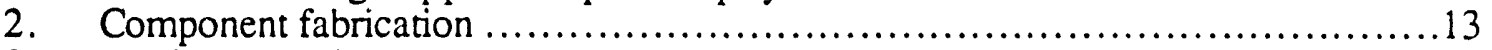

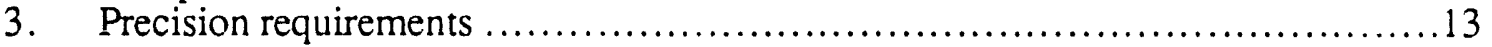

4. Manufacturing technology development requirements............................13

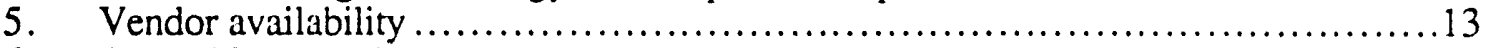

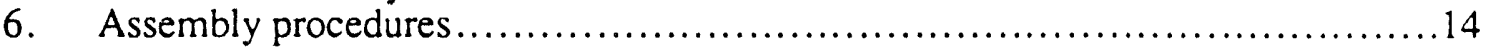

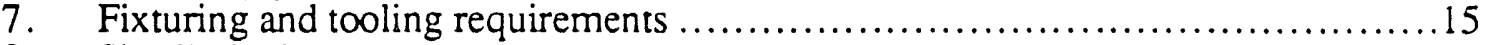

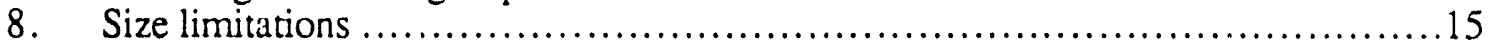

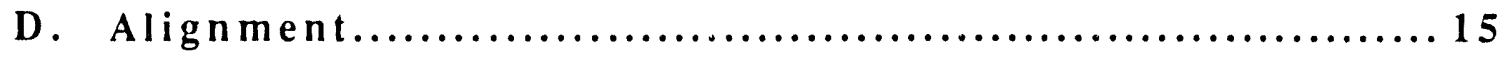

E. Structural Performance.................................... 16

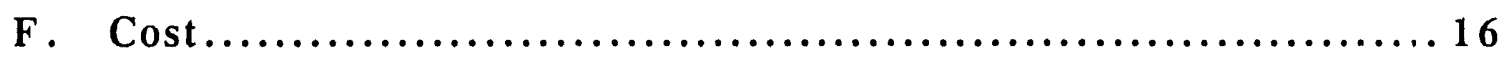

1. Assumptions, basis ...................................................... 16

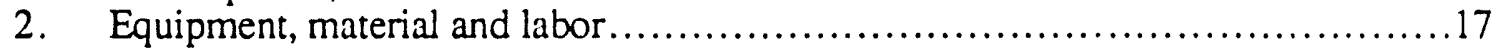

3. Development, engineering, procurement/fabrication, ...........................22

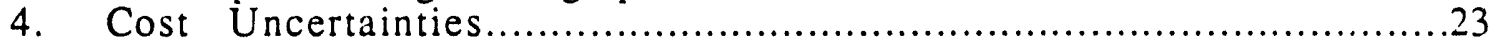


G . Schedule................................................. 23

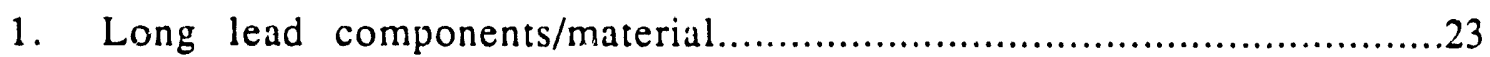

2. Component Fabrication Time ...................................................... 23

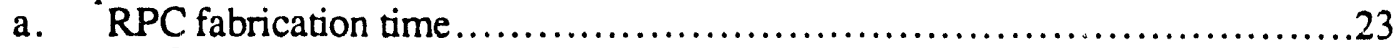

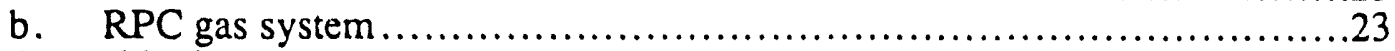

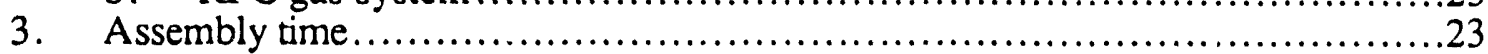

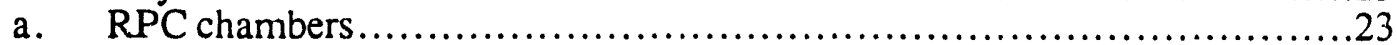

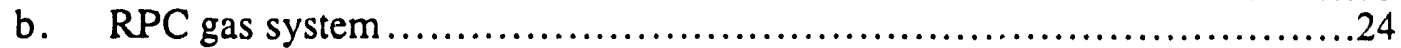

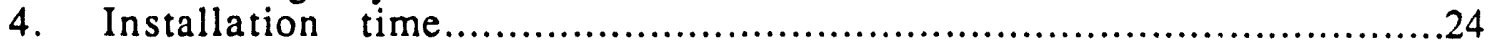

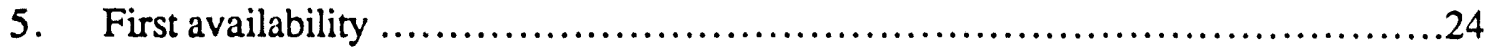

H . Strength of Supporting Group............................ 24

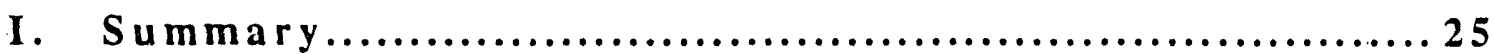

J. General References ...................................... 26

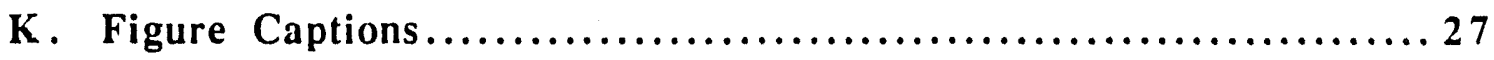

Appendix 1. Presentation to the Muon Trigger Meeting, 22 January 1992 by Prof. I. A. Pless.

Appendix 2. Meantimer (chronotrons) technique for use with Resistive Plate Chambers by Prof. D. Marlow.

Appendix 3. "Behavior of Large Resistive Plate Counters," Draft GEM Note, September, 1992. 


\section{A. Design}

\section{General Design Concept}

Resistive Plate Chambers (RPCs) have been in operation since 1981. The current standard (Italian) design is two meters long by one meter wide with a sensitive gas gap of 2 millimeters. There are no wires or conductors anywhere in the sensitive region. The walls of the chamber - in contact with the gas of the sensitive volume - consists of a semi-conductor (Bakelite, for example) with a bulk resistance of about $10^{11} \mathrm{ohm}$-centimeters. An ionizing particle passing through the sensitive volume $(2 \mathrm{~m} \times 1 \mathrm{~m} \times 2 \mathrm{~mm})$ breaks down the gap and creates an electrical discharge. This discharge is very fast (a few nanoseconds). The discharge is capacitively coupled to pick-up strips which are located outside the semiconductor walls of the gas cell.

When the strip is terminated with a 50 ohm load the voltage pulses across the termination are typically 0.5 volts high with a rise time of $2-3 \mathrm{~ns}$. A typical full width at half height (FWHM) is about $10 \mathrm{~ns}$ and the measured rise time jitter is less than $1.4 \mathrm{~ns}$. Figure 1 is a schematic of the "standard" Italian RPC.

RPCs are used to perform the fast triggering necessary to identify the SSC beam bunch crossing associated with a particular physics event, as well as the muon momentum trigger (Level 1 trigger) for identifying muons of sufficient momentum. Bunch crossing identification is performed using straight line fits of tracks registered on projective strips oriented in the phi direction (non-bend plane), with widths of $3.9,6.5$, and $8.9 \mathrm{~cm}$, for inner, middle, and outer super-layers, respectively. Muon momentum measurements are made using strips of $1.3 \mathrm{~cm}$ width oriented along the $z$ direction (bend plane). The strip widths are chosen to provide the necessary precision for track identification. In the case of the bunch-crossing identification, the wide strips are all that is necessary to identify a straight line track. In the case of the muon momentum identification, $1.3 \mathrm{~cm}$ strips are sufficient to provide sagitta measurements of the necessary precision to identify muons of particular momenta, for example, $>10 \mathrm{GeV} / \mathrm{c}$, or $>50 \mathrm{GeV} / \mathrm{c}$. This momentum identification is based on the measurement of the muon passage through a set of strips in the three super-layers and the strip displacement from a straight line as projected from the origin through the outer super-layer strip.

Identification of tracks in an RPC is a simple matter of reading the induced charge on the cathode strips, identifying the time of arrival of the charge, and the spatial coordinates of the strip. The RPC operation (spark chamber), implies that it is essentially a digital device. The critical conditions for RPC operation are 1) stability of materials used in the RPC, 2) stability and uniformity of the gas supplied to the RPC, and 3) uniformity of the gas gap within the RPC that gives rise to the spark breakdown and the subsequent induced pulse on the pick-up strips. The RPC system, being a somewhat less mechanically precise system than the proposed drift tube systems, uses much simpler manufacturing methods than the precision drift tube technologies being considered for the GEM Muon System.

\section{Standard RPC}

The Bakelite plates in a standard Italian RPC are $2 \mathrm{~mm}$ thick. As stated above, the gas gap is also $2 \mathrm{~mm}$ thick. The edges are sealed with a strip of PVC $1 \mathrm{~cm}$ in width. The di- 
mensional tolerances are modest. The thickness of the Bakelite plate is irrelevant for the proper operation of the RPC. The combined flatness and spacing requirements between the plates are \pm 200 microns. This requirement is trivial to maintain over arbitrarily large areas by using PVC spacers $2 \mathrm{~mm}$ thick and $11 \mathrm{~mm}$ in diameter spaced on a $10 \mathrm{~cm}$ grid. The $2 \mathrm{~m}$ $x 1 \mathrm{~m}$ chambers have been tested by raising one corner with respect to the two opposite corners by $10 \mathrm{~cm}$. There was no measurable change in the performance in this warped geometry as compared to the performance when the chamber is flat. The chambers have been stood up with the $2 \mathrm{~m}$ edge vertical and then allowed to fall flat. After this mishandling the chamber worked exactly as before the fall.

Hundreds of these chambers have been produced and are operating in experiments all over the world. A set of these chambers have operated at Frascati for 7 years. Such chambers are currently being used in E-771 at Fermilab. Recently L3 at CERN has ordered 400 of these chambers from General Technica, Colli, Italy, which is a company that is a commercial supplier of RPCs.

This history has been related here to emphasize that we are not talking about a new technology, but rather a mature technology which is well understood and for which the manufacturing technology has already been transferred to industry.

\section{Weight and other Design Parameters}

Figure 2 is a sketch of our two gap RPC design. As stated in the General Design Concept (Section 1), there are spacers that maintain the sensitive gas gap distance. These spacers produce about a $1 \%$ dead area. The two gap design we have chosen eliminates this $1 \%$ dead area by having two gas gaps with the spacers in one gap staggered with respect to the spacers in the second gap. This double gap constructions also has the virtue of providing practically $100 \%$ redundancy for the RPC system.

Table 1, GEM RPC Specifications contains, among many other items, the detailed breakdown of the weights. Note that we have assumed 0.015" (380 micron) thick Bakelite plates rather than $2 \mathrm{~mm}$, as this choice of thickness could increase the repetition rate (at $95 \%$ efficiency, typical for an RPC operating at plateau) from $\sim 100 \mathrm{~Hz} / \mathrm{cm}^{2}$ to $\sim 1000$ $\mathrm{Hz} / \mathrm{cm}^{2}$.

Note that from Table 1 one can calculate that the weights of the individual chambers are $70 \mathrm{lbs}, 104 \mathrm{lbs}$, and $146 \mathrm{lbs}$ for nominal radii of 4.0,6.0, and $8.0 \mathrm{~m}$ (inner, middle and outer super-layers), respectively.

Hence any chamber can be easily handled by four technicians (one for each corner) without the use of cranes or special hoisting apparatus.

\section{Wire or Strip Support}

As stated previously, there are no sense wires in this technology. The pick-up strips are formed on the aluminized mylar. There are several well-known technologies for this purpose. The simplest method utilizes a shadow mask as mylar is aluminized. In addition, etching, grinding, and sand-blasting are all viable techniques for creating the strips. The required precision for the strips is \pm 100 microns. Once the mylar is glued into the RPC stack, the strip positions need to be known with respect to an outside fiducial with a precision of $\pm 2 \mathrm{~mm}$. 
Table 1. GEM RPC Specifications

Coverage: $\quad 100 \%-\mathrm{Z}, 95 \% \mathrm{Phi}$

Areal Mass: $\quad$ projected thickness $=0.7 \%$ Xo/layer

Material Thickness Rad. Length \% Rad. Length

$\begin{array}{llll}\text { Al } & 0.005 " & 8.9 & 0.14 \\ \text { Foam } & 4 \mathrm{~mm}, & 424 & 0.09 \\ \text { Mylar } & 0.005 " & 28.7 & 0.04 \\ \text { Bakelite } & 0.015 " & 34.4 & 0.11 \\ \text { Gas } & 2 \mathrm{~mm}, & \text { Large } & 0 \\ \text { Bakelite } & 0.015 " & 34.4 & 0.11 \\ \text { Mylar } & 0.005^{\prime \prime} & 28.7 & 0.04 \\ \text { Foam } & 4 \mathrm{~mm} & 424 & 0.09 \\ \text { Al } & 0.005 " & 8.9 & 0.14 \\ \text { Foam } & 4 \mathrm{~mm}, & 424 & 0.09 \\ \text { Mylar } & 0.005 " & 28.7 & 0.04 \\ \text { Bakelite } & 0.015 " & 34.4 & 0.11 \\ \text { Gas } & 2 \mathrm{~mm} & \text { Large } & 0 \\ \text { Bakelite } & 0.015 " & 34.4 & 0.11 \\ \text { Mylar } & 0.005 " & 28.7 & 0.04 \\ \text { Foam } & 4 \mathrm{~mm} & 424 & 0.09 \\ \text { Al } & 0.005 " & 8.9 & 0.14 \\ \text { Total: } & & & \\ & & & 1.38\end{array}$

Nominal Radius (M):

4.0

64

148.5

330.0

631.9

114

166

1.3

3.9

Width of non-bend-plane strips $(\mathrm{cm})$ :

Weights (lbs):

Bakelite:

Aluminum perimeter frame:

Cable:

Connectors, brackets:

TOTAL/Super-layer
1877

1049

385

1160

4471

TOTAL WEIGHT (TONS) 16.5 TONS 
Strips are an integral part of the mylar, therefore, the strip support is the mylar base. The mylar, in turn, is part of the RPC laminate.

\section{RPC Gas System}

LLNL is designing the gas system. The envisioned system is a low-flow, simple stateof-the-art recirculating system using standard technology that has been tested and proven for many years. All components are standard, off-the-shelf items.

The RPC muon trigger system covers a total 2743 square meters of surface area in the three muon super-layers and contains $0.4 \mathrm{~cm}$ total thickness of gas layers. This corresponds to a total gas volume of 10.9 cubic meters. Typical RPCs operate with a gas flow rate of about $10 \%$ of their volume per hour or about 1.1 cubic meters per hour (18.3 liters per minute) for this system. The RPC gas system is designed to provide this flow rate for an accurately mixed combination of gases.

Previous RPC systems have run successfully using a gas mixture consisting of $66 \%$ Argon, 32\% n-butane and 2\% freon. It is unlikely that this mixture of gases will be allowed at the SSC because of its flammable nature. Alternative gas mixtures are being explored in $\mathrm{R} \& \mathrm{D}$. One candidate gas is a non-flammable mixture of $49 \% \mathrm{CO}_{2}, 49 \% \mathrm{CF}_{4}$, and $2 \%$ freon.

Figure 3 shows one idea for the design of a gas system for mixing 4 different gases. This system is designed for explosive mixtures but is entirely suitable for non-explosive mixtures as well. Component gases are introduced into separate lines and combined in accumulators using precision mass-flow controllers and proportioning valves. All valves and controls are designed for fail-safe operation, e.g. valves will fail in closed positions and excess pressures are bled off into special vent lines into accumulators in the event of a power outage. Much of the gas system is designed to allow the proper inlet and outlet pressures to the precision flow controllers for proper metering of gas.

Mass flow controllers such as the MKS 1159B series are used. A separate mass flow controller, with a nominal range suitable for the particular gas, is used for each component gas. These controllers are interfaced to 8 channel power supply/readout modules with an IEEE-488 (GPIB) computer interface.

Figure 4 shows a sketch of a gas delivery system that could be used for the RPC system. Each RPC module will have gas inlets and outlets (1, 2, and 3 each, for inner, middle, and outer super-layer modules, respectively). The inlet and outlet number increases from the inner super-layer to the outer super-layer because the RPC module width increases by about 1 meter per super-layer. To insure good flow characteristics, while allowing for reasonable pressures, the gas system splits the flow into the outer and middle super-layers and circulates from the central membranc outward. The inner super-layer circulates the gas from end to end. This insures uniform gas flow in different size modules. All tubing and fittings are metal (copper or stainless steel tubing, stainless steel or regular steel swage-lok fittings) for fire safety.

Exhaust gas will not be allowed to be vented to the atmosphere because of the admixture of freon. Exhaust gas will be fed into a gas recovery system whose design is yet to be determined. This system will likely contain an accumulation tank and a compressor system that takes the exhaust gas and compresses it through various pressure stages to condense the lowest vapor pressure gases. In this case the first stage condenses freon gas to a liquid, 
which is collected for disposal or recirculation. Likewise, the remaining $\mathrm{CO}_{2} / \mathrm{CF}_{4}$ mixture is further compressed with the $\mathrm{CO}_{2}$ being the next gas to liquefy and be collected. All gas components can be reintroduced into the input stage or properly discarded at this point.

\section{Chamber Structure}

The chamber structure will follow the well-tested and proven Italian design. The chamber will be made up of various layers glued together to form a single self-supporting laminated plate. Spacers, consisting of $2 \mathrm{~mm}$ thick, $11 \mathrm{~mm}$ diameter PVC disks serve three functions. First, they mechanically separate the two Bakelite plates and keep the plates flat with respect to one another by being placed on a $10 \mathrm{~cm}$ square grid. Second, they insulate the resistive plates from each other. Third, they transmit the mechanical stresses from one side of the plate to the other.

The edges of the RPCs will be sealed by gluing $1 \mathrm{~cm}$ wide, $2 \mathrm{~mm}$ thick PVC strips between the Bakelite plates. This stiffens the edges mechanically so that the plate is selfsupporting. In addition, there will be an aluminum U-channel frame that will encompass the chamber on all four sides. This aluminum U-channel frame will be glued to the completed RPC body. The purpose of this frame is, first, to protect the edges of the RPC during shipping, handling and installation; second, to stiffen the edges so that the RPC can be mounted by four corners (if necessary); and third, to furnish the base to mount the high voltage connectors, the gas connectors and the LEMO signal connectors.

All the above technology is well understood and presents no engineering or manufacturing problems. There is a problem of availability of wide sheets of Bakelite. Bakelite sheet comes in arbitrarily long lengths, but the standard width is 48 inches. This concern will be addressed in an upcoming section.

\section{Impact on truss structure design}

The weight of the largest RPC Chamber is 146 lbs. This weight should have a negligible impact on the truss structure design. Because the dimensional tolerances of the RPC are so loose, the RPC can be fastened directly to the drift chamber systems. Hence, they should introduce no geometrical impact on the truss structure design.

\section{Super-layer configuration}

The RPC system is broken up into three levels: the RPC itself, the RPC chamber, which encloses two layers of the RPC, and the RPC super-layer, of which there are three; inner, middle, and outer. The inner, middle and outer RFC super-layers form a complete RPC sector, with 16-fold symmetry in phi, and mirror symmetry about the z-axis of the GEM detector. Figures 5 - 9 show various sketches and drawings of the system

The RPC itself consists of two layers of detectors, slightly staggered within a single chamber enclosure. This staggering is necessary to prevent trigger inefficiency due to the presence of spacers and other hardware that contribute to dead area in the RPC.

The RPC chamber is a box which encloses the two layers of RPC and is envisioned as an aluminum frame with honeycomb sheet covers. RPC chamber widths and lengths vary 
$148.5 \mathrm{~cm} \times 330 \mathrm{~cm}, 239.0 \mathrm{~cm} \times 360 \mathrm{~cm}$, and $329.6 \mathrm{~cm} \times 380 \mathrm{~cm}$, for inner, middle, and outer super-layer chambers, respectively.

Each super-layer consists of overlapping RPC chambers, again, to increase trigger efficiency. The inner, middle and outer super-layer sectors are made up of two, three and four overlapping RPC chambers, respectively.

In summary, there are 64 inner sector chambers, 96 middle sector chambers and 128 outer sector chambers with a total projected surface area of 2743 square meters.

Table 1 provides estimates the weight of the chambers in each super-layer sector including the RPC itself, the RPC chamber enclosure with aluminum perimeter frame, RPC perimeter cable weight with an additional $10 \%$ added in each super-layer for connectors, brackets, etc.

Summarizing, the inner super-layer weight is $4471 \mathrm{lb}$., the middle super-layer weight is $9911 \mathrm{lb}$, and the outer super-layer weight is $18655 \mathrm{lb}$. The total weight for the RPC subsystem is 16.5 tons for the entire GEM detector RPC system, or about 1 ton per sector.

\section{Electronic Packaging Requirements}

As presently designed, there will be no electronics inside the magnet. All that is required is high voltage connectors, gas connectors and LEMO connectors for strip signals to be carried on RG-174/U 50 ohm coaxial cable.

\section{Size Limitation}

As already mentioned, the widths of the RPC presents a concern. Bakelite comes in arbitrarily long lengths but in standard widths of 48 inches. Since the total amount of material needed by GEM is small, it is unlikely that we can afford to purchase special machinery for our widths. However, this avenue will be explored. Fortunately, due to the natural design of an RPC, it is very simple to "splice" several narrower sheets to create one "wide" sheet.

As an example, we look at the largest chamber, which is $329.6 \mathrm{~cm}$ wide Since our Bakelite is $121.92 \mathrm{~cm}$ wide, two splices are needed. Figure 10 illustrates a possible splice design. The scale is about 10:1.

The critical feature of the splice is that it is recessed into the Bakelite plate. The two edges facing the gas are hand-sanded to remove any burrs. As in the standard construction of RPCs, all inner surfaces are coated with linseed oil after assembly. This oil is quite viscous and will fill in the 400 micron or so crack and cover any glue that oozes up through the crack. A visual inspection will be made to insure that the glue doesn't not protrude above the surface of the Bakelite. This will be controlled before the chamber is laid out. Note that the splices on the upper and lower plates do not line up so that the distortion of the electric field in the region of the splice is kept to a minimum. Note also that the electric field at the splice is somewhat lower than the normal electric field. This has two consequences. The first is that there is a smaller likelihood of a field breakdown in the vicinity of the splice. Hence the splice should not be a source of noise. The second is that the efticiency will be somewhat lowered in the neighborhood of the splice. If we assume that the efficiency is zero for the 400 microns of each of the two splices, we find this corresponds to a loss of $0.02 \%$ efficiency of the overall chamber. This is totally negligible. Of course 
there will be the need of special jigs and tooling to make these splices, but this is a very straight-forward manufacturing problem that contains no basic engineering difficulties.

\section{Utility routing}

As noted above, the only utilities for the RPC are the high voltage, gas, and signal cables. The high voltage and gas require two high voltage cables per chamber and two, four or six gas lines per inner, middle, and outer chamber, respectively. These obviously present no routing problem whatsoever.

However, the signal cables do present a problem. This problem was discussed in detail at the 22 January, 1992 Muon Group Meeting at SSCL in Dallas. The following paragraphs are from that presentation.

Note that in this report it is assumed that RG58/U, 0.193" diameter coaxial cable would be used. We have since decided to use RG174/U coaxial cable, which has a diameter of 0.101 ". Therefore each linear routing dimension of the 22 January report can be reduced by a factor of 0.52 . For the square geometry in the cable harnessing, we will have bundles with cross sections 2.7", 3.9", and 8.3" on a side. For the rectangular geometry we will have cable bundles of 1.1 " thick with widths of 8.8", 14.7" and 66". Either the square geometry or the rectangular geometry can be accommodated in the current truss structure design. The choice will depend on multiple scattering studies. The square geometry has moderate multiple scattering over a few inches, while the rectangular geometry has a large multiple scattering over 1.1 inches.

\section{Trigger}

A muon trigger should furnish at least two pieces of information. They are:
A. Beam crossing tag.
B. Momentum cut of the muon which created the trigger.

The RPC technology is ideally suited for tagging the beam crossing time. The characteristics of the RPC and cable routings are as follows:

1. Rise time jitter is measured to be less than 1.4 nanoseconds everywhere on a 1 $\mathrm{m} \times 2 \mathrm{~m} \mathrm{RPC}$.

2. By proper choice of cable routing, all signals from a beam crossing come to a common data collection point within a fixed time gate of eight nanoseconds. However, knowing which strips have been hit allows one to calculate the beam crossing time to $1.4 \mathrm{~ns}$, the rise time jitter of the RPC pulse.

There are three straightforward ways to take advantage of these characteristics in forming a trigger which furnishes the beam crossing time and the muon momentum cut.

1. Discrete components and pipeline fan-in. Appendix 1 discusses this technique in detail. 
2. Field-programmable gate array devices. A preliminary study indicates that the objectives of the trigger can be met with standard field programmable gate array devices.

3. Mean timers, or "chronotrons." Professor D. Marlow has proposed a simple analog scheme. This scheme is contained in Appendix 2.

In summary, given the fast rise time and small rise time jitter of an RPC pulse, it is straightforward to generate a first level momentum selection trigger and to identify the proper beam crossing.

\section{Timing and trigger widths}

If we collect all signals from the strips in one corner of the chambers and inatch cable propagation speeds to strip propagation speeds then signals are matched in time when they arrive at the chamber corner. At the Level 1 trigger, we know the beam crossing to better than $12 \mathrm{~ns}: 8 \mathrm{~ns}$ due to the $1.49 \mathrm{~m}$ length of the strip and $4 \mathrm{~ns}$ due to the rise time jitter of the RPC pulse. This latter number has to verified by R\&D, however, measurements made on the $1 \mathrm{~m}$ strips of the Italian RPC indicate time jitter of about $1.4 \mathrm{~ns}$.

Referring to Figure $11 \mathrm{a}, \mathrm{b}$; at the time of the first level trigger, the identity of the $\mathrm{x}$ and y strips are known. At this point, one can calculate the beam crossing time to an accuracy of the rise time jitter of the RPC. For track 1, the signal from the outer layer arrives $9 \mathrm{~ns}$ after the signal from the inner layer and $4.5 \mathrm{~ns}$ after the signal from the middle layer. For track 2, the signal from the outer layer arrives $13 \mathrm{~ns}$ before the signal from the inner layer and $6.5 \mathrm{~ns}$ before the signal from the middle layer. Hence the trigger widths on the outer layer must be $13 \mathrm{~ns}$ long, while the trigger widths on the inner layer must be $9 \mathrm{~ns}$ long and the trigger widths on the middle layer must be $6.5 \mathrm{~ns}$ long.

The first question with respect to the hardware is how does one get the signals out to the common electronics point? In our case we propose to bring the signals out along the edge of the RPC layers at the layer level and along the 60 degree line to the outer layer for the inter-layer connections. For each layer we have 161 cables running along the edge of the layer. Using RG-58 cable, this corresponds to a bunch 5.8 inches on a side. For the run from the inner layer to the central layer we use 274 cables. This is a cable bundle 7.5 inches on a side. For the run from the middle layer to the outer layer we have 1,264 cables. This cable bundle is 16 inches on a side.

Alternately, we can run all cables in a flat package two inches wide. This would give widths for $17,28.2$, and 128 inches wide for along the layer, from the inner layer to the middle layer, and from the middle layer to the outer layer, respectively

\section{Failure tolerance}

As stated previously, the RPC is a very rugged reliable device without sense wires or regions of high fields. However, failures do occur. In case of a failure one half of the double RPC would fail. Because of this possibility, we operate each RPC gap as a separate chamber. The loss of one RPC gap means a decrease of efficiency of $1 \%$. This is negligible from any practical point of view. Therefore we can state that the RPC system is, for practi- 
cal purposed, $100 \%$ redundant and that any single failure has a negligible effect on the practical efficiency of the system.

Another way of looking at this situation is to suppose on gap fails per year. This is more than two orders of magnitude higher failure rate than is indicated by our current experience. Under this assumption one can show that the probability of two gaps failing in the same RPC chamber is ten years of operation is less than $10 \%$.

\section{B . RPC R\&D Results}

RPC R\&D has been carried out using a multi-faceted approach with the goal being to completely characterize the RPC operation and applicability to the GEM Muon System. R\&D has focused on studying the existing state-of-the-art as exemplified by the standard Italian design already described, as well as new RPCs using non-standard materials and fabrication methods, with an emphasis on reduced mass, stability and long term performance, and increased rate capability.

\section{Standard Italian and Glass RPC Performance}

MIT and LLNL have now studied a number of different RPC designs, including a $1 \mathrm{~m}$ $x 2 \mathrm{~m} \mathrm{RPC}$ provided by R. Santonico of the University of Rome, glass RPCs, cermet thin film RPCs and RPCs using a number of low resistivity materials such as static-dispersive plastics. Extensive testing of the Italian RPC and a custom built glass RPC has been carried out at MIT, including measurements of efficiency, rise-time jitter, and response to neutrons and the results of these tests are detailed in Appendix 3. LLNL has also performed similar measurements on-a smaller glass RPC.

In summary, the MIT and LI.NL glass RPC (resistivity $=5 \times 10^{12} \mathrm{ohm}-\mathrm{cm}$ ), with its relatively high resistivity has a reduced rate handling capability and a reduced efficiency (70\% for the MIT RPC with $2 \mathrm{~mm}$ glass and $90 \%$ for the LLNL RPC with 750 micron glass). The Italian RPC (resistivity $=1-2 \times 10^{11} \mathrm{ohm}-\mathrm{cm}$ ) exhibits about $95 \%$ efficiency using cosmic rays and a scintillator telescope for triggering. The Italian RPC has a measured saturated counting rate of about $560 \mathrm{~Hz} / \mathrm{cm}^{2}$ compared to a rate of about $50 \mathrm{~Hz} / \mathrm{cm}^{2}$ for the MIT glass RPC ( $75 \mathrm{~Hz} / \mathrm{cm}^{2}$ for the LLNL glass RPC). On the other hand, the these different RPCs agree remarkably well in terms of pulse characteristics, e.g., pulse height, pulse width, rise time and pulse velocity along the strip.

\section{Trigger Jitter}

Trigger jitter measurements on the Italian RPC give of the order of $1 \mathrm{~ns}$. The MIT glass RPC exhibits a larger jitter of about 7 ns due to the inability to achieve an adequate high voltage across the gas gap as detailed in Appendix 3.

\section{Neutron Sensitivity}

Neutron sensitivity measurements were made using a strong Cf-252 source. The sensitivity of the RPC to $1-10 \mathrm{MeV}$ neutrons is measured to be $4.8 \times 10^{-3}$ and to $1-10 \mathrm{MeV}$ photons to be $6.6 \times 10^{-3}$. 


\section{RPC Theory}

A related effort has been carried out at LLNL to understand the theory of operation of RPCs in order to make better selections for resistive materials that can substantially increase the rate capability of the RPC. The RPC can be modeled as an equivalent circuit as shown in Figure 12. This circuit has been used as the basis for SPICE modeling of the RPC response for different resistive materials. This response is characterized by a recovery time that is dependent on the resistive properties of the material used in the RPC, as well as the capacitance of the different sections. Figure 13 shows the recovery time expected for a number of different RPC materials. As can be seen, the recovery time can vary over a large range depending on the material. This recovery time is directly related to the saturated rate capability of the RPC as discussed below.

The following is a simple model that lets one deduce the rate of a counter at a fixed efficiency given the saturation counting rate. This model usually underestimates the rate for high efficiencies.

$$
\begin{aligned}
& \text { Assume: } \\
& R s=\text { saturation rate } \\
& \operatorname{Re}=\text { rate at a fixed efficiency } E \\
& \operatorname{Re}=\operatorname{Rs}(1-E)
\end{aligned}
$$

This equation is trivial to derive but it is only an approximation as it assumes a linear relation between saturation counting rate and the period of inefficiency of the counter. Note that this equation predicts that there is no rate for which a counter can be $100 \%$ efficient, which is true as every counter has to have a dead time and regardless of rate there is a nonzero probability to have two random counts within this dead time. If one assumes $E=$ 0.95 , which is the number that is normally used for this equation, then for $\mathrm{Rs}=20,000 \mathrm{~Hz}$ we can assume that the counter will operate at $1,000 \mathrm{~Hz}$. Because of the approximation previously mentioned, this derived rate is actually an underestimate of the real rate.

\section{Alternative RPC Materials - High Rate RPCs}

RPCs have been fabricated at LLNL using sputtered resistive cermet thin films on thin glass or plastic substrates. We have demonstrated that Cermets can function as RPCs however the long term aging characteristics of these thin films is not clear. Indications are that the films are not strongly bonded to the substrate and are subject to sputtering effects because of ion bombardment. Alternative materials to Bakelites have been explored, concentrating on static-dispersive plastics with low bulk resistivities in the range $10^{8}-10^{11} \mathrm{ohm}$ $\mathrm{cm}$. We have successfully demonstrated RPCs with a number of different plastics. Table 2 summarizes the properties of these materials along with the Bakelites and glasses.

We have measured the saturated counting rates from two different static-dispersive plastics, Mitech-411 and Abstat-M310. For the case of the Mitech plastic, our SPICE model predicts a saturated counting rate capability of about $2.3 \times 10^{4} \mathrm{~Hz} / \mathrm{cm}^{2}$. We have assembled a small RPC with this material and in fact measure a count rate of approximately $1.5 \times 10^{4} \mathrm{~Hz} / \mathrm{cm}^{2}$. Figure 14 plots the counting rate versus high voltage for an RPC made 
with Mitech-411. This rate has not been determined to be saturated and further work is in progress to determine the absolute saturated count rate of the RPC with this very interesting material.

Figure 15 plots the rate capability of these various RPCs as calculated by SPICE versus the measured count rate. The SPICE calculated rate capability is arbitrarily chosen to be the inverse of the half-height point on the recovery curve for each material. As can be seen in Figure 15, the calculation is simply related to the experimental measurements by a linear relation. The confirmation of the SPICE model by this result indicates that the properties of the RPC are dominated by the bulk resistivity of the plates and not by the surface resistivity.

This count rate is substantially faster that any other standard RPC materials measured to date, and constitutes a significant improvement in the state-of-the-art. In addition, the Abstat plastic has a significantly higher dielectric strength and is available in thinner sheets than chose used in this measurement (720 microns) that could potentially give an increase of 5 - 10 times the rate of the present detector.

\section{Table 2. RPC Resistive Materials Properies}

\section{Material Thickness $(\mathrm{cm})$ Bulk Resistivity $(\Omega-\mathrm{cm}) \operatorname{Arc}$ Resistivity $\left(\Omega-\mathrm{cm}^{2}\right)$}

$\begin{array}{llll}\text { MIT mirror glass } & 0.300 & 5.00 \times 10^{12} & 1.50 \times 10^{12} \\ \text { LLNL mirror glass } & 0.066 & 4.90 \times 10^{12} & 3.23 \times 10^{11} \\ \text { Kodak projector glass } & 0.123 & 6.42 \times 10^{11} & 7.89 \times 10^{10} \\ \text { Italian RPC Bakelite } & 0.200 & 1.00 \times 10^{11} & 2.00 \times 10^{10} \\ \text { LLNL Bakelite } & 0.161 & 4.50 \times 10^{9} & 7.24 \times 108 \\ \text { Abstat-M310 plastic } & 0.072 & 5.78 \times 10^{9} & 4.16 \times 10^{8} \\ \text { Abstat-M310 plastic } & 0.060 & 5.78 \times 10^{9} & 3.47 \times 10^{8} \\ \text { Mitech-411 plastic } & 0.090 & 2.03 \times 10^{9} & 1.83 \times 10^{8} \\ \text { Mitech-411 plastic } & 0.030 & 2.03 \times 10^{9} & 6.19 \times 10^{7} \\ \text { Corning 0211 glass } & 0.056 & 6.70 \times 10^{7} & 3.75 \times 10^{6} \\ \text { Boron film } & 0.0001 & 1.00 \times 10^{6} & 1.00 \times 10^{2}\end{array}$

\section{Lifetime Testing of the Italian RPC}

Lifetime (aging) tests have also been performed using the Italian $1 \mathrm{~m} \times 2 \mathrm{~m}$ Bakelite RPC. We chose an area of this RPC approximately $5 \mathrm{~cm} \times 10 \mathrm{~cm}$ whicn was irradiated with a radioactive source. The counting rate on this area was approximately $250 \mathrm{~Hz} / \mathrm{cm}^{2}$. We placed a cosmic ray scintillation counter telescope which had approximately the same 5 $\mathrm{cm} \times 10 \mathrm{~cm}$ area over the chosen area of the RPC. We chose the RPC region so that one of the PVC spacers was contained in the area.

We define one SSC year (at a luminosity of $10^{33}$ ) to be $2 \times 10^{7}$ counts per square centimeter. This is the expected neutron interaction rate at the $10^{33}$ luminosity. We accumulated $16.4 \times 10^{7}$ counts per square centimeter in our test, which is equivalent to $8.2 \mathrm{SSC}$ standard years. The data is shown in Figure 16.

Before irradiation the chosen area had an efficiency of $93.6 \pm 3 \%$. The fact that the measured efficiency is not about $97 \%$ is a reflection of the mismatch between the cosmic 
ray hodoscope and the RPC area. After an irradiation equivalent to 8.2 SSC years of operation, we measured the efficiency of this area to be $92.4 \pm 3 \%$. As can be seen from Figure 16, within the $3 \%$ errors, we find no change in efficiency for the equivalent 7 SSC year exposure at a luminosity of $10^{33}$.

\section{RPC Manufacture/assembly}

\section{Manufacturing approach/philosophy}

As stated in the introductory section, identification of tracks in an RPC is a simple matter of reading the induced charge on the cathode strips, identifying the time of arrival of the charge, and the spatial coordinates of the strip. The RPC operation (spark chamber), implies that it is essentially a digital device. The critical conditions for RPC operation are 1) stability of materials used in the RPC, 2) stability and uniformity of the gas supplied to the $\mathrm{RPC}$, and 3) uniformity of the gas gap within the RPC that gives rise to the spark breakdown and the subsequent induced pulse on the pick-up strips.

Our manufacturing philosophy is therefore to build as large a chamber as possible for each super-layer without compromising the performance necessary to achieve the above stated goals. This implies that the materials used in the individual chambers should be able to be assembled into a self-supporting structure that first and foremost maintains the gas gap dimensions with a tolerance of about $\pm 5 \%$, or in the case of a ? mm gas gap, \pm 100 microns. Similar dimensional tolerances are necessary for the distance between the pick-up strip electrodes and their ground plane, in order to control the strip impedance. The gas gap is maintained by gluing $2 \mathrm{~mm}$ thick, $1 \mathrm{~cm}$ diameter disk spacers every $10 \mathrm{~cm}$ or so in between the laminated sheets. The strip-electrode-to-ground plane gap is maintained by virtue of the rigid foam to form the proper impedance transmission line. Because of this design flexibility the RPC can actually be deformed substantially from a flat plane and still operate correctly.

As an extreme case, the RPC could be formed into a series of concentric cylindrical shells rather than the flat sectors envisioned in GEM and theoretically provide the same operating characteristics. Thus the structural support for the RPCs can be minimized to help reduce muon scattering. The RPCs in GEM can be allowed to deform by as much as $10 \mathrm{~cm}$ over the largest dimensions without affecting the overall operation of the muon trigger system, although proper support structure design can probably minimize this sag to less that 1 $\mathrm{cm}$. Because materials associated with the RPC should be minimized, the addition of support frames will probably not be allowed. Also, depending on the design, RPCs could be integrated into the drift tube structure, utilizing this structure to minimize sags.

Similarly, the tolerance associated with the placement of the pick-up strips is defined by the desired measurements of timing (bunch crossing) and momentum (sagitta). This tolerance as we see it implies strips to be located also to about \pm 100 microns, a not too difficult tolerance to achieve using standard machining or photolithography techniques.

Materials stability is an issue for long term performance of the RPCs. Plastics degrade over time due to out-gassing of plasticizers, interaction with ambient UV light, temperature fluctuations, radiation, and, in the case of RPCs, uniformity and stability of the bulk resistivity of the resistive plates and interaction of the plates with spark discharges. Proper materials choices and design will help to insure the long term stability of the RPCs. We can 
benefit from the experience of other RPC systems that show long term (years) operation of RPCs without degradation, albeit, under lower rate conditions than that expected at the SSC. Aging tests are easily performed in R\&D to determine the proper long term operation of RPC materials.

\section{Component fabrication}

Component fabrication will be based on commercially available materials of more-orless standard sizes in order to keep costs to a minimum. For example, Westinghouse can provide Bakelite of a standard width of 4 feet (or less - they can cut to any specified width in this range) and with a length of many meters. Bakelite sheets would be cut to the necessary lengths for the particular RPC chamber dimension and then butted together with adhesives while cross laminated with aluminized mylar sheet for strength. The assembly of RPC chambers is a relatively simple process of laminating layers of material together into a final structure that is then incorporated into a perimeter frame containing gas and electrical connections. In addition this frame allows for attachment points for the RPCs to the precision tracking chambers or to the superstructure of the Muon System.

Other components for the RPCs include rigid foam board, aluminized mylar, glues, PVC spacers (1 cm diameter, $2 \mathrm{~mm}$ height) for maintaining the gas gap, gas fittings (swage-lok), electrical connectors (LEMO or Kings K-lok), high voltage connectors (SHV or GHV), and extruded aluminum channel. All these components are commercially available. Fabrication of the RPC laminates requires large area benches of approximately $4 \mathrm{mx}$ $5 \mathrm{~m}$ at the largest for the outer super-layer.

\section{Precision requirements}

There are no precision requirements necessary for the RPC assembly. Standard tolerances on thicknesses of Bakelite and other commercially available laminate materials are sufficient for the proper operation of the RPCs. The PVC disk spacers can be turned out using computerized machining with the necessary height tolerance of \pm 100 microns $( \pm 4$ mils) without difficulty. Pick up strips can be laid out by photoetching aluminized mylar sheet or by machining with fine cutting tools. Either method is capable of providing the necessary dimensional tolerance of \pm 100 microns.

\section{Manufacturing technology development requirements}

There are no major technology development requirements needed to manufacture RPCs, except perhaps, fixturing for holding the RPCs during storage, testing, shipping and assembly into the Muon System.

\section{Vendor availability}

RPCs have been manufactured in large quantities by Italian University groups for many years. In fact, we have performed a large part of our R\&D on an Italian RPC with dimensions $1 \mathrm{~m} \times 2 \mathrm{~m}$ at MIT. A factory to build these RPCs is already in place and is capable of building RPCs to our specifications with minor modifications to their manufacturing pro- 
cess to take into account our new materials. If the need arises the manufacturing of RPCs could be carried out in the US at LLNL or MIT or also in China. Tsing Hua University in Beijing has written a Letter of Intent with the GEM Detector Collaboiation to perform a number of manufacturing services for the various sub-systems including electronics for calorimeter and muon systems, as well as structural ccinponent assembly for calorimetry and the muon system.

\section{Assembly procedures}

288 modules in three different sizes are needed to make a complete system. Table 1 lists the module dimensions, and other parameters. The modules are seen to have non-standard dimensions and will have to be made from smaller sized pieces. The joining of pieces of rigid foam board, for example, will be accomplished by utilizing the specially coated mylar films as cross-plies glued on each side of the boards. Additional rigidity will come from the joining of the mylar/foam laminates through the gluing of the small gas gap spacers. The overall laminate will be somewhat flexible but will not exhibit any substantial shear deformation.

Figure 17 shows a detail of the RPC where the laminate layers join the perimeter frame. Refer to Figure 18 for identification of the various materials in the RPC laminate. There is an insulating margin of $1 \mathrm{~cm}$ around the perimeter of each RPC laminate layer to allow the attachment of the laminate to aluminum U-channel without shorting the cermet electrodes or the edge pick-up strips. The laminate layers fit into $1.3 \mathrm{~cm}$ wide grooves machined in the U-channel and a bead of glue is applied around all edges to provide a gas-tight seal. Gas feed-throughs $(2,3$, or 4 for inner, middle, and outer super-layers, respectively) are attached on the outside of the end perimeter frame in-line with the RPC gas gaps. There are two redundant layers of RPC per module and each layer of pick-up strip electrodes are daisy-chained together internally. Pick-up strip electrodes are connected to LEMO bulkhead connectors in the perimeter frame using short lengths of wire.

The perimeter frames are assembled with three sides welded together. The RPC laminates are guided into the perimeter frame grooves and the fourth perimeter frame piece is attached and glued, rather than welded. Holes in the perimeter frame are also provided for mounting brackets. If additional stiffness is required, carbon composite or aluminum cross beams can be installed.

Figure 19 shows an assembled half-sector of RPCs. The RPC modules overlap in $\mathrm{Z}$ in order to provide $100 \%$ coverage for muon trigger efficiency. $100 \%$ overlap in phi may not be possible because of structural supports between the sectors. In this case the phi coverage is about $95 \%$. As mentioned previously, there are two redundant RPC layers per module. These layers are interconnected internally for both bend-plane and non-bend plane strips. Module-to-module daisy-chaining is done in the $Z$ direction ( $1.3 \mathrm{~cm}$ bend plane strips) to effectively form single strips spanning the length of each half-sector. These interconnects are made using short lengths of RG-174A/U (50 ohm) coaxial cable. Non-bend plane strips are distributed to individual RG-174 coax cables along the longitudinal sides of the RPCs. These cables are bundled are routed out to the trigger processing electronics at the ends of the RPC system. 


\section{Fixturing and tooling requirements}

Special tooling is not required for the RPC system. Electrical connectors use standard machine threads for bulkhead feed-throughs, gas fittings use standard pipe threads. Perimeter frames of aluminum are welded or glued together. Laminates are glued using epoxies. Precision spacers for maintaining the gas gap can be turned out using computer controlled machining techniques. Large area plastic sheets with strip electrodes $n d$ to be laid out either by machining strips in a solid aluminized mylar sheet or by photolithography methods.

Fixturing for supporting the RPC units during shipping, storage and assembly will need to be designed. A simple fixture would support the RPC vertically to prevent undue stress on the unsupported panel and would have wheels for allowing the chamber to be moved.

\section{Size limitations}

The envisioned RPC design is constrained to sized less than $4 \mathrm{~m}$ in length for ease of handling. Our philosophy is to minimize the number of chambers for simplicity in the gas system and electrical interconnects. The current configuration of $2-3-4$ chambers per super-layer do not match the presently envisioned configuration for the precision tracking chambers (2 - 4 - 4), however, the RPC chamber oesign is flexible and can be re-designed to overlap whatever final tracking chamber configuration is decided upon. RPCs have a low mass of about 0.5 psi and so a fully assembled RPC chamber will weigh about 70 lbs., $100 \mathrm{lbs}$, and $150 \mathrm{lbs}$ for a single inner, middle, and outer chamber, respectively. The weights and dirnensions are within reason for 2 or 3 technicians to handle a completed chamber using light duty chain hoists or overtiead cranes.

\section{Alignment}

Because of the previously mentioned performance criteria for RPCs as a Muon System trigger, precision alignment is not necessary for these chambers. The RPCs can be mounted either separately from the precision drift tube technology, or co-located with the drift tubes. It is assumed during construction that sufficient care in fabrication will allow strips to be located with an accuracy of about $0.1 \mathrm{~mm}$ with respect to a fiducial placed on the edge of the RPC support frame. Sag in the RPC will be within the limits imposed for proper muon timing and momentum determination so that monitoring of the sag will not be necessary.

In-situ RPC alignment can be performed using cosmic rays or physics events at the SSC. In addition, if the RPC system is incorporated into the drift tube structure, alignment will be coupled to the drift tube alignment system without added cost. This is because the RPC strip readout is referenced to a fiducial that can be referenced to the drift tube alignment system. In any event, RPC location to $1 \mathrm{~mm}$ in all degrees of freedom is sufficient to insure proper operation for Level 1 triggering. 


\section{E. Structural Performance}

A first attempt at modeling the RPC structural performance has been made at LLNL. A standard Italian RPC was modeled using the following assui nptions:

1. RPC sandwich assumed to be $3000 \mathrm{~mm} \times 4000 \mathrm{~mm} \times 2 \mathrm{~mm}$ Bakelite sheets with $10 \mathrm{~mm}$ diameter, $2 \mathrm{~mm}$ thick disk spacers every $10 \mathrm{~cm}$.

2. Alunninum $6061-t 6$ frame with cross-stiffeners.

3. Support fixed at four comers.

4. Sandwich bonded to frame and stiffeners.

5. FEA model has stiffeners on one side only but with $2 x$ section modulus.

$$
\text { 6. Bakelite properties: } \quad E=1.239 \times 10^{4} \mathrm{~N} / \mathrm{mm}^{2} \text {, density }=1.87 \times 10^{-5} \mathrm{~N} / \mathrm{mm}^{3}
$$

The results of the analysis are shown in Figure 20. Deflection under the RPC's own weight is $109 \mathrm{~mm}$. Frame stress is less than $25 \mathrm{ksi}$. The fundamental frequency is estimated to be less than $10 \mathrm{~Hz}$.

The next set of calculations will examine the RPC for the two layer design with thinner Bakelite layers. These calculations indicate that the stresses to not appear to limit the design of the largest RPC chamber. The deflection is likely to be reduced, in the case of 0.015 " Bakelite, to about $10 \%$ of the deflection seen in this calculation. In addition, there is ample room to increase stiffener cross-sections and to increase frame heights. If the RPC is mounted on the drift chamber, then calculations will be needed to establish the deflection of the drift chamber due to the added load of the RPC. It is likely that this added load will not adversely effect a properly designed drift chamber system.

\section{F. Cost}

\section{Assumptions, basis}

The cost of the RPC system has undergone a series of refinements starting with studies begun in October, 1991. In this time two addition cost studies have been performed, culminating in the Cost Estimate prepared on June 2, 1992 after experiences with the May 5, 1992 cost review held at SSCL. This costing was performed for both the Bakelite design and an alternative Cermet thin film design that held promise for reduced weight. R\&D on the Cermet concept is continuing, however there is sufficient indication that thin Bakelite or plastic sheets of the proper resistivity can offer the desired performance and weight reductions without resorting to the exotic thin film technologies needed to fabricate Cermets. The June 2 costing reflects a Bakelite system using $2 \mathrm{~mm}$ thick sheets of Bakelite material for the resistive plates. Thinner sheets, for example 15 mil Bakelite, might be suitable and provide a reduction in the cost. In addition the cost of structural support will certainly be reduced as the RPC system is made lighter. We assume a cost of $\$ 300 / \mathrm{m}^{2}$ for this structure 
although a detailed design for the structure has not yet been made. The June 2, 1992 costing memo follows (with modifications based on reduced structure cost).

\section{Equipment, material and labor}

Based on our experience with the Cost Review of May 5, 1992, we have attempted to revise our cost estimate to reflect new design changes and also to incorporate more "realistic" cost estimates based on vendor quotes.

Design changes have occurred that radically alter the RPC concept. This is based on new experience with the "Italian" RPC now in testing at MIT. It has been realized that a rigid, gas-tight box of aluminum honeycomb is not necessary. In addition, a new cabling scheme has cut the amount of cabling substantially.

In the process of revising the cost estimates a couple of errors were uncovered in the costing of Bakelite sheet and rigid foam sheet. The errors were in the calculation of the total volume of material needed and they have been corrected in this new Cost Estimate. Foam sheet is substantially cheaper in this estimate than in the previous (First Order) estimate, due to a change in the foam to Styrofoam, and the cost was determined by contacting a local plastics supplier. The cost of Bakelite sheet was confirmed verbally by contact with Westinghouse sales representatives to be the same as in the LLNL stock catalog.

For all estimates we have now included stainless steel tubing and swage-lok fittings, LEMO connectors, and cabling to the edge of the magnet. Additionally, we assume that the electronics cost is specified by the Trigger and Data Acquisition Group cost estimate

We estimate the total projected surface area of the RPC system to be $2743 \mathrm{~m}^{2}$ consisting of two (somewhat redundant) concentric layers of RPCs in each super-layer.

With our more accurate description of the RPC system, we find that the cost has fallen substantially from our previous First Order estimate: \$2M for the Bakelite RPC system (no electronics cost included) compared to our "zeroth order" estimate of about $\$ 6 \mathrm{M}$ for a $2,000 \mathrm{~m}^{2}$ system (which included the cost of electronics). So the cost of the RPC system will be about $\$ 700$ per square meter (the RPC itself is about $\$ 200 / \mathrm{m}^{2}$ ). The breakdown of the cost is as follows:

\section{Standard Bakelite RPC design}

Item

Cost $\left(\$ / \mathrm{m}^{2}\right)$ Total Cost $(\$ K)$

1. Aluminum U-channel

$4.3 \quad 11.8$

2. Bakelite sheet or Cermet coated plastic film

3. Foam (e.g. polyurethane sheet)

27.0

74.1

4. Aquadag

1.2

16.9

3.2

5. Aluminized Mylar sheet

15.4

46.4

7. Spacers, glue

0.36

Total Materials

65.16

42.2

1.0 
Total Materials (x.25 for mach'g

Cost $\left(\$ / \mathrm{m}^{2}\right)$ Total Cost $(\$ \mathrm{~K})$

$81.45 \quad 223.4$

and waste):

Item

Cost $\left(\$ / \mathrm{m}^{2} \perp\right.$ Total Cost $(\$ \mathrm{~K})$

8. Fabrication cost estimate: $\$ 60 / \mathrm{hr} @ 1 \mathrm{~m}^{2} / \mathrm{hr}$

9. Gas fittings

$60.0 \quad 164.6$

edge $\mathrm{x} 2$ for gas manifold)

10. Steel tubing (1/4" diameter, $10.4 \mathrm{~km}$ long) $11.52 \quad 31.6$

11. RG-174 coaxial cabling $(67.5 \mathrm{~km}) \quad 5.18 \quad 14.2$

12. LEMO connectors $(2 \times 33,184$ channels $) \quad 80.0 \quad 219.4$

13. Gas (Recirculator)

Total Miscellaneous

$21.14 \quad 250.0$

$252.4 \quad 692.4$

\section{Subtotal:}

Materials, Fab., Gas system, Electrical system:

\section{Cost $\left(\$ / \mathrm{m}^{2} \perp\right.$ Total Cost $(\$ K)$}

333.9
915.8

Thus the total cost is $\$ 1 \mathrm{M}$ for a $2,743 \mathrm{~m}^{2}$ system. We will take the cost of electronics to be $\$ 36 /$ channel $\times 33,184$ channels $=\$ 1.19 \mathrm{M}$, although this cost does not appear in the WBS worksheets. We will conservatively estimate the cost of any RPC specific structural support to be $\$ 300 / \mathrm{m}^{2}$ which is probably a gross overestimate and so zero contingency is assumed for this. Contingency for materials is set to be $5 \%$ and contingency for labor is assumed to be $100 \%$,

\section{Cost $\left(\$ / \mathrm{m}^{2}\right)$ Total Cost $(\$ K)$}

RPC Materials, Fab., Gas system

Structural supports and integration with drift tubes

Materials contingency ( $5 \%$ )

Labor contingency $(100 \%)$
333.9

300.0

15.85

60.00
915.8

823

43.5

164.4

\section{Cost $\left(\$ / \mathrm{m}^{2} \perp\right.$ Total Cost $(\$ \mathrm{~K})$}

\section{TOTAL}

709.8

1,947

Thus for a $2743 \mathrm{~m}^{2}$ system, the total cost would be about $\$ 2 \mathrm{M}$. The largest uncertainty is in the structural support and integration cost estimate.

The two Cost Estimate Worksheets: WBS \# 3.2.1.2.4.1.3 and WBS \# 3.2.1.2.4.1.5 have been revised to reflect these new costs. Percentages are broken down differently than was done in the last worksheet to reflect what we think is a more realistic percentage for Materials, Machining and Fab/Ass'y. The total Materials cost is now about $\$ 98.25 / \mathrm{m}^{2}$. Taking 25\% of this number for machining and waste (our previous estimate of $100 \%$ of this number now seems somewhat high) gives $\$ 25 / \mathrm{m}^{2}$ and we still use $\$ 60 / \mathrm{m}^{2}$ for Fab/Ass'y. This gives the percentages: $54 \%, 13 \%$, and $33 \%$, respectively. Which is used for the basis of estimates in WBS \# 3.2.1.2.4.1.3 and WBS \#3.2.1.2.4.1.5. 


\section{MUON SYSTEM \\ COST ESTIMATION WORKSHEET}

WBS Element Title: Central Region Sector Assembly; RPCs; Off-Site; Assembly

WBS Element No: 3.2.1.2.4.1.3 Date: 11 Sep 92 Rev: 3 Estimator: T. Hamilton, C. Wuest

Scupe: This element covers the labor and labor cost for the assembly of the RPC chambers, and the purchase and labor cost for assembly equipment.

\begin{tabular}{llll}
\hline Engineering/Design & PY: & Comp. Rate(\$K/PY): & PoP: \\
N/A & &
\end{tabular}

M\&S: Engineering/Design and Inspection/Administration (\$K): 14

Misc. office supplies for Administration:

PC/Mac/workstation charyes:

Inspection/Administration

PY: 2.25 Comp. Rate(\$K/PY): 133 PoP: 7/94-6/96

Assumes Eng (nat'l avg) oversight of assy equipment purchase activity: 1 eng, full-time for 3 mos = .25 PY from Weinstein/Osborne estimate: ('cost of production')

- reduced to $76 \%$ (for certial region only), actually time-scaled reduced from 36 to 24 mos (67\%)

- Eng (nat'l avg) oversight of assembly activity: 1 eng, full-time for 2 years $=2.00 \mathrm{PY}$

Procurement/Fabrication Material

LSDT/Weinstein/Osborne estimate was $\$ 365 \mathrm{~K}+\$ 50 \mathrm{~K}$ misc

- assumed misc assy equip:

- assumed misc nuts/bolts, pins, etc.:
$(\$ K): 300$

$\$ 250 \mathrm{~K}$

$\$ 50 \mathrm{~K}$

Instailation/Assembly

PY: 6.0 Comp. Rate(\$K/PY): 112 PoP: 7/94-6/96

LSDT/Weinstein/Osborne estimate was 1 PY Eng, 1 PY Sr. Tech for machine set-up

- assumed same for RPC estimate

from Wuest/Pless estimate:

- $\$ 60 / \mathrm{m}^{2} \times 2743 \mathrm{~m}^{2}=\$ 165 \mathrm{~K} / \$ 107 \mathrm{~K} / \mathrm{PY}$ (sr. tech, nat'l avg)

-for assembly: Wuest/Pless estimate of $\$ 300 / \mathrm{m}^{2}$ was prorated against materials, machining, and assembly based on percentages established on Page 3 of Wuest/Pless "Second Order" estimate: $\$ 300 / \mathrm{m}^{2} \times 2743 \mathrm{~m}^{2} \times 33 \%=\$ 272 \mathrm{~K} / 107 \mathrm{~K} / \mathrm{PY}$

$2.5 \mathrm{PY}$

Material: Installation/Assembly

Misc. office supplies for Install/Assembly:
$(\$ K): 6$

$\$ 6 \mathrm{~K}$ 
Contingency

Technical:

Cost:

Schedule:
0 Basis: none

30 Basis: engineering judgment, labor rate \& material concern

8 Basis: jelays completion of critical path item

\section{Comments}

Because all costs were given in $\$ / \mathrm{m}^{2}$, all labor rates/categories were assumed to be national average. This assumption is reflected in the PY labor loading. If lower rates were assumed by Wuest/Pless, the labor loading would be higher. 


\section{MUON SYSTEM \\ COST ESTIMATION WORKSHEET}

WBS Element Title: Central Region Sector Assembly; Bakelite RPCs; Off-Site; Machining/Inspection WBS Element No: 3.2.1.2.4.1.5 Date: 11 Sep 92 Rev: 5 Estimator: T. Hamilton, C. Wuest

Scope: This element covers the labor and labor costs for the machining and inspection of the Bakelite RPC and assembly fixturing. Also, costs for oversight and purchasing of stock and material for machining.

\begin{tabular}{llll}
\hline Engineering/Design & PY: & Comp. Rate(\$K/PY): & PoP: \\
N/A
\end{tabular}

M\&S: Engineering/Design and Inspection/Administration (\$K): 45

Misc. office supplies for Inspection/Administration:

$\$ 15 \mathrm{~K}$

PC/Mac/workstation charges:

$\$ 22 \mathrm{~K}$

Travel: 1 person, 4 trips to machine shop, inspection shop @ \$2K/trip

$\$ 8 \mathrm{~K}$

Inspection/Administration

PY: 6.19 Comp. Rate(\$K/PY): 72 PoP: 1/94-3/96

Assumes Engineering (nat'l avg) oversight of stock/material purchase activity: $1 / 4$-time for 24 mos=.50 PY Assumes Engineering (nat'l avg) oversight of mach'g \& inspect activity: $1 / 2$-time for $2.25 \mathrm{yrs}=1.13 \mathrm{PY}$ Assumes Sr. Tech (job shop) performing inspection activity: 1 tech, full-time for $2 \mathrm{yrs}=2.00 \mathrm{PY}$ Assumes Jr. Tech (job shop) performing inspection activity: 1 tech, full-time for 2 yrs $=2.00$ PY

- no inspection cost estimate provided from Wuest/Pless

- estimate of $\$ 100 \mathrm{~K}$ (approx. $2 \mathrm{PY}$ ) for inspection from Weinstein/Osborne estimate approx doubled

Procurement/Fabrication Material

(\$K): 900

from Wuest/Pless "Second Order" estimate: Wuest/Pless cost estimate multiplied by $2743 \mathrm{~m}^{2}$ for central region

Aluminum U-channel:

$\$ 12 \mathrm{~K}$

Bakelite sheet $(2 \mathrm{~mm})$ :

$\$ 74 \mathrm{~K}$

Foam sheet $(2 \mathrm{~mm})$ :

$\$ 3.2 \mathrm{~K}$

Aquadag:

$\$ 46 \mathrm{~K}$

Aluminized mylar $\left(0.005^{\prime \prime}\right)$ :

$\$ 42 \mathrm{~K}$

spacers, glue, etc:

$\$ 1.0 \mathrm{~K}$

material for structural support:

$\$ 444 \mathrm{~K}$

- for structural support material: Wuest/Pless "First Order" estimate of $\$ 600 / \mathrm{m}^{2}$ was prorated against materials, machining, and assembly based on percentages established on Page 3 of Wuest/Pless "First Order" estimate : $\$ 300 / \mathrm{m}^{2}$ x $2743 \mathrm{~m}^{2}$ x $54 \%=\$ 444 \mathrm{~K}$

- general M\&S (nuts, screws, gas fittings, elect. connectors):

$\$ 232 \mathrm{~K}$

- fixtures stock (ss tubing, coax cable):

$\$ 46 \mathrm{~K}$ 
from Wuest/Plcss "First Order" estimate:

\section{RPCs Machining}

1.) $\$ 25 / \mathrm{m}^{2} \times 2743 \mathrm{~m}^{2}=\$ 69 \mathrm{~K} / \$ 74.5 \mathrm{~K} / \mathrm{PY}=1.0 \mathrm{PY}$

2.) for structural support machining: Wuest/Pless estimate of $\$ 300 / \mathrm{m}^{2}$ was prorated against materials, machining, and assembly based on percentages established on Page 3 of Wuest/Pless "Second Order" estimate $\$ 300 / \mathrm{m}^{2} \times 2743 \mathrm{~m}^{2} \times 54 \%=\$ 444 \mathrm{~K} / \$ 74.5 \mathrm{~K} / \mathrm{PY}=6.0 \mathrm{PY}$

Assumed assembly fixtures: 2 machinists, full-time for 1 year: 2 PY

$$
=9.0 \mathrm{PY} \text { total }
$$

Material: Installation/Assembly

Misc. office supplies for Installation/Assembly:
$(\$ K): 18$

$\$ 18 \mathrm{~K}$
Contingency

Technical:

Cost:

Schedule:

\section{0}

30

8

\section{Total: 38}

Basis: none

Basis: engineering judgment, labor rate \& material concern

Basis: delays completion of critical path item

\section{Comments}

3. Development, engineering, procurement/fabrication, installation/assembly

Research and development will likely continue to refine the RPC with emphasis on reduction of materials (for increased muon resolution and decreased structural cost). In addition, new materials that show promise for high rate applications in the expected neutron background are being studied at the present time. These materials are typically low resistivity plastics that are commercially available with similar costs to Bakelite, however the manufacturers have greater flexibility to provide these materials in odd sizes and non-standard thicknesses with little impact on cost.

Engineering is being carried out at LLNL to determine the structural requirements of the RPCs and will likely continue in coordination with engineering efforts at Draper Labs and SSC Laboratory.

Procurement will be carried out by coordinators at LLNL and SSCL and fabrication of RPCs can take place overseas if cost of labor is an issue. Installation/assembly, of course, takes place at SSCL and requires similar conditions for installation/assembly of drift tube technologies, although the individual chamber weights are not as demanding on personnel and facilities. Estimates of manpower for these items are given in the Cost Estimation Worksheets in the previous section. 


\section{Cost Uncertainties}

The major cost uncertainty at this time is the structure cost, because the RPC may be incorporated into the drift tube structure and utilize its interface to the super-structure. In addition, the cost of machining and fabrication is somewhat uncertain. Also, electronics costs are not included in this review and estimates vary from about $\$ 12 /$ channel to about $\$ 35 /$ channel depending on the source of the electronics and the fabrication in the US or overseas. Cost of materials is more certain, given the standard nature of the materials involved. Cost estimates for materials have been based on manufacturer's quotes or LLNL stock book prices for fittings, connectors, plastics, glues, etc. Details of costs have been presented in a number of reviews over the past year and can be found in various Cost Review proceedings.

In summary, it appears that a complete RPC system can be built for a cost of less than $\$ 3 \mathrm{M}$. This cost can perhaps be further reduced by a simplification of the structural requirements if the RPCs are incorporated in the drift tube support system.

\section{G. Schedule}

\section{Long lead components/material}

All material and components are off-the-shelf stock items. Once the items have been identified, all items and materials can be at the RPC Factory witnin 120 days of receipt of purchase orders.

\section{Component Fabrication Time}

\section{a. RPC fabrication time}

If we assume a team of four people, we estimate that construction can proceed at a rate of about 160 square meters per week. Our average chamber has an area of 10 square meters. Hence, we can estimate that this assembly team can assemble about 16 chambers per week or about three chamber per day. The total of 288 chambers will therefore take, under this assumption, 18 weeks. We have added a factor of 2 in our contingency for this item. In this case, the 36 week estimate for fabrication time would fit into any envisioned SSC/GEM schedule.

\section{b. RPC gas system}

All the components of the gas system are off-the-shelf items, hence there is really no component fabrication time.

\section{Assembly time \\ a. RPC chambers}

Once the RPC chambers are fabricated there is no further assembly time. 
b. RPC gas system

Gas systems, such as the RPC system, usually take about six months to assemble. We now have gained experience in assembling a 4 component gas system at LLNL and feel reasonably confident in this estimate.

\section{Installation time}

The installation time of the RPC system is completely dictated by the drift chamber installation time. Given the weight of the RPC and the alignment tolerance of the RPC, the RPC system will not slow dow'n the drift chamber system installation time.

\section{First availability}

From this point in time, we still need a one year final $R \& D$ effort to construct the largest prototype RPC chamber. This chamber would be constructed by the members of the RPC Collaboration. It would then take a second R\&D year to set up a proper factory including special jigs and handing tools, and to work out the assembly line bugs. At the end of that year the first production line chambers would be produced. Given the appropriate $R \& D$ funding for those two years, the first production RPC chamber would appear in January, 1996.

\section{H. Strength of Supporting Group}

The RPC collaboration consists of a number of physicists, engineers and technicians from 6 institutions and are listed at the beginning of this report. We have demonstrated a strong multi-disciplinary R\&D effort in FY 1992 in fields of RPC theory, design, fabrication, and characterization, materials science, structural engineering, and costing. We draw on our experience from a number of different programs in high energy physics, nuclear physics, applied physics, chemistry and materials science, mechanical engineering, and electronics engineering. MIT and LLNL both maintain laboratories exclusively dedicated to RPC research and development. In addition, a number of special purpose facilities are available. For example, MIT has facilities for measurement of RPC behavior in magnetic fields. LLNL maintains a number of electron linear accelerators for beam testing of RPCs. LLNL also has an extremely strong mechanical engineering support group already in place for the GEM Muon system with expertise in designing very large systems, CAD/CAM, structural engineering, systems integration, costing, and mass production techniques. Both LLNL and MIT have very strong shop support for prototyping large chambers. We have fostered contacts with overseas institutions such as ITEP in Moscow and Tsing Hua University in Beijing, as well as contacts at the highest levels of the Chinese Academy of Sciences which could be useful for mass production of chambers with a substantial cost savings in labor. 


\section{Summary}

We summarize this report by reiterating a number of important facts about RPCs:

A. They are the only Muon System technology currently made by industry. The standard sized RPC is one meter by two meters. In principle sizes much larger than this can be built.

B. They have the following pulse characteristics:

1. Pulse height about 0.5 volts without amplification into 50 ohms.

2. Pulse rise time less than 5 nanoseconds.

3. Pulse width about 10 nanoseconds.

4. $\quad$ Measured rise time jitter less than 1.4 nanoseconds (no other Muon System technology has this speed).

C. Lifetime is greater than $16.4 \times 10^{7}$ pulses per square centimeter with no loss of efficiency within the $3 \%$ measuring error. This is equivalent to 8.2 standard SSC years (at luminosity $10^{33}$ and $2 \times 10^{7}$ seconds per year - the expected neutron flux is $2 \mathrm{~Hz}$ per square centimeter.)

D. Commercial RPCs have demonstrated essentially $100 \%$ efficiency at about at about $100 \mathrm{~Hz}$ per square centimeter using standard Bakelite resistive plates. This can be compared to CSCs, which can only tolerate, for electronic reasons, $2 \mathrm{~Hz}$ per square centimeter.

E. RPC prototypes built with new lower resistivity materials have demonstrated non-saturated counting rates of about $20,000 \mathrm{~Hz}$ per square centimeter, with an expectation of a factor of 5 - 10 further increase by utilizing thinner materials.

F. Refinement of the RPC design has led to an extremely low' mass system with minimal muon scattering cross section of about $0.7 \% \mathrm{X}_{\mathrm{o}}$ /layer.

G. A series of cost reviews carried out over the past year indicate that the cost of the complete RPC system for GEM is about $\$ 2 \mathrm{M}$ not including the cost of the electronics.

As part of our planned R\&D program in the next year, we will measure RPC lifetimes out to $10^{9}$ pulses per square centimeter. This corresponds to approximately 5 standard SSC years at a luminosity of $10^{34}$. We plan to build an larger prototype RPCs up to 3 meters by 4 meters. The demonstrated improvement in rate capability of a large RPC from 100 $\mathrm{Hz} / \mathrm{cm}^{2}$ to over $1000 \mathrm{~Hz} / \mathrm{cm}^{2}$ is a significant result and there are indications that with the proper combination of low resistivity materials and plate thicknesses, a factor of 10 or more increase in rate corresponding to $10^{4}-10^{5} \mathrm{~Hz} / \mathrm{cm}^{2}$ is achievable in RPCs.

Based on the R\&D we have performed, along with a detailed analysis of the cost of constructing a full RPC trigger system for GEM, we feel that the combination of pressur- 
ized drift tubes (PDTs) for precision tracking coupled to RPCs for fast Level 1 triggering has the least risk, the highest muon resolution, the best beam crossing timing accuracy, and the lowest cost of all competing technologies.

\section{J. General References}

1. G. Battistoni, et al., "Plastic Spark Counters with PVC Electrodes," Nucl. Inst. Meth. A270, p. 190, 1988.

2. R. Cardarelli, et al., "Progress in Resistive Plate Counters," Nucl. Inst. Meth. A263, p. 20, 1988.

3. M. Della Negra, et al, "Study of Muon Triggers and Momentum Reconstruction in a Strong Magnetic Field for a Muon Detector at LHC," CERN/DRDC/90-36 DRDC/P7, August 30, 1990.

4. M. Anelli, et al., "Glass Electrode Spark Counters," Nucl. Inst. Meth. A300, p. 572, 1991.

5. "An Expression of Interest to Construct a Major SSC Detector," B. Barish, W. Willis, Co-spokespersons, pps. 3-38 - 3-40, July, 1991, unpublished.

6. C. Bacci, et al., "Muon Tracking and Hadron Punchthrough Measurements using Resistive Plate Chambers," Preprint ROM2F/91/18, July 22, 1991.

7. A. Bohrer, et al., "Status Report of the RD5 Experiment," CERN/DRDC/91-53, January 13, 1992. 


\section{K. Figure Captions}

1. Sketch of Italian (single layer design) Resistive Plate Chamber.

2. Schematic layout of materials in the proposed two layer RPC for the GEM Muon System. See Table 1 (p. 3) in the text for the corresponding radiation length fractions.

3. Four component gas mixing system designed and built at LLNL. A similar system will be utilized for the RPC system in GEM.

4. Schematic layout of the gas delivery system for the RPC chambers. Note that the gas is split at the central membrane and directed to the ends of the sectors for the outer and middle super-layers. The inner super-layer directs the gas from end to end.

5. Representation of an RPC/drift chamber sector concept using a 3-3-2 configuration.

6. Detail of RPC attachment concept.

7. Schematic of inner RPC super-layer showing dimensions and $Z$ overlaps.

8. Schematic of middle RPC super-layer showing dimensions and $Z$ overlaps.

9. Schematic of outer RPC super-layer showing dimensions and $Z$ overlaps.

10. Sketch of the bonding of two Bakelite (or similar material) panels. The upper splice is filled in with epoxy and the linseed oil coating covers the bond. Cross plies of aluminized mylar further strengthen the bond.

11. Sketch showing the trigger timing concept. In a) the key is to match strip propagation speeds with coaxial cable propagation speeds (by matching their characteristic impedances) so that signals in $\mathrm{X}$ and $\mathrm{Y}$ arrive at a chamber corner at the same time. In b) the signals are fed out to the sector 60 degree edge and then routed out of the magnet.

12. Equivalent circuit of the RPC used in SPICE to predict the operating characteristics of the RPC. The RPC is modeled as a capacitor and a resistor for each plate coupled via a capacitor, corresponding to the gas gap. The calculated RPC characteristics include recovery time, which is directly correlated to the saturated counting rate capability of: the RPC.

13. SPICE calculation results for different RPCs. In this figure we compare a number of different materials: Bakelite, glass, and plastics.

14. Experimental measurement of counting rate versus high voltage for an RPC fabricated with Mitech-411 plastic. Note that the vertical scale is in counts $/ \mathrm{second} / 2 \mathrm{~cm}$. Thus this RPC has a peak counting rate of about $1.5 \times 10^{4} \mathrm{~Hz} / \mathrm{cm}^{2}$. 
15. Comparison of SPICE calculated RPC rate capability with measured rates for a number of different RPCs. The RPCs plotted are, in order of increasing rate, the MIT glass, the LLNL glass, the Italian Bakelite, the Abstat-310, and the Mitech-411. The linear fit to the points is also indicated. The rate capability is defined as the inverse of the half-height time of the curves in Figure 13.

16. Results of lifetime measurements performed on the Italian RPC. The data is consistent with no degradation in performance for an equivalent of 8.2 SSC years of operation, assuming a $2 \mathrm{~Hz} / \mathrm{cm}^{2}$ rate at a luminosity of $10^{33}$.

17. Concept sketch showing the RPC laminates bonded to an aluminum $U$ channel perimeter frame. Also shown are gas and signal connectors. Refer to Figure 18 for details of the laminated layers.

18. Detail of RPC laminates in Figure 17.

19. Sketch of an assembled half-sector of RPCs. The RPC modules overlap in $\mathrm{Z}$ in order to provide $100 \%$ coverage for muon trigger efficiency. $100 \%$ overlap in phi may not be possible because of structural supports between the sectors. In this case the phi coverage is about $95 \%$. There are two redundant RPC layers per module. These layers are interconnected internally for both bend-plane and non-bend plane strips. Module-to-module daisy-chaining is done in the $\mathrm{Z}$ direction $(1.3 \mathrm{~cm}$ bend plane strips) to effectively form single strips spanning the length of each half-sector. Nonbend plane strips are distributed to individual RG-174 coax cables along the longitudinal sides of the RPCs. These cables are bundled are routed out to the trigger processing electronics at the ends of the RPC system.

20. Displacement contours for a $3 \mathrm{~m} \mathrm{x} 4 \mathrm{~m}$ Bakelite RPC supported at its corners. The maximum displacement is about $10.9 \mathrm{~mm}$ in the center of the panel. Details of the calculation are given in the text on p. 15. 


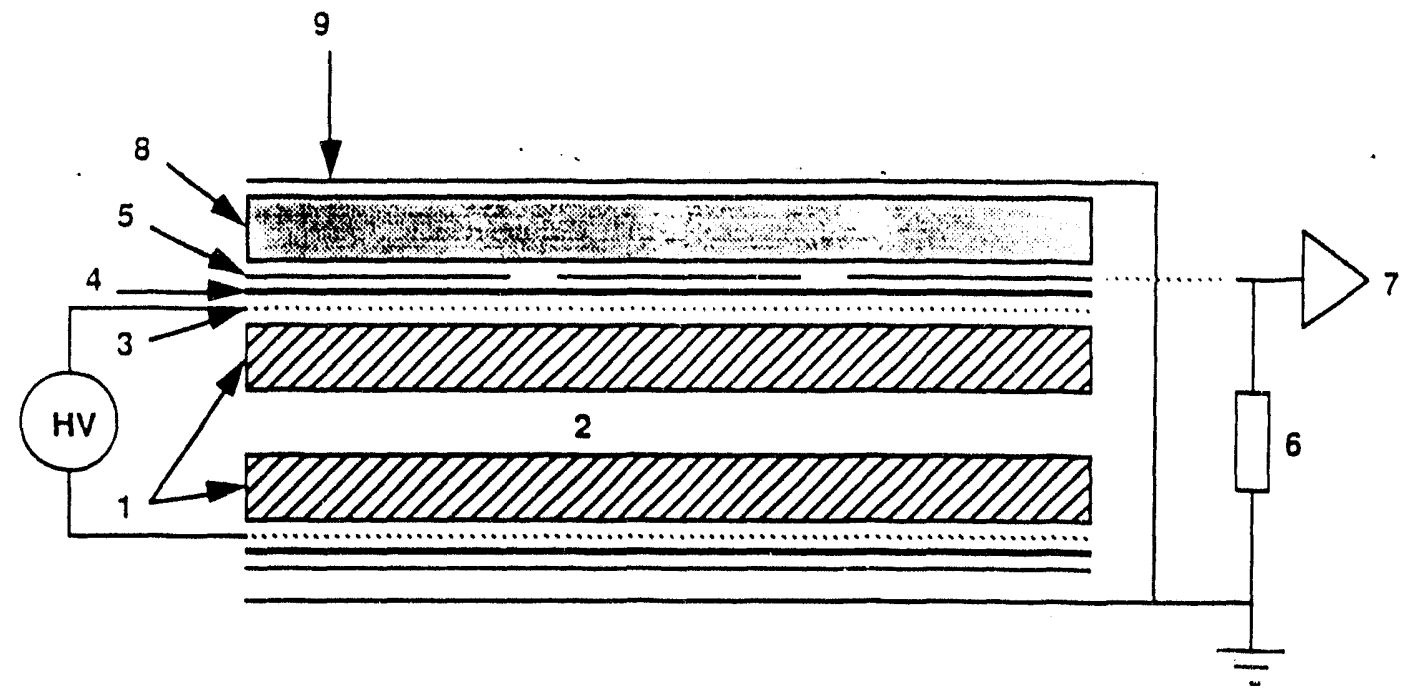

1 Bakelite plate

2 gas

3 graphite coating

4 insulating foll

5 aluminum strips

6 line termination

7 discriminator

8 foam

9 aluminum ground plane

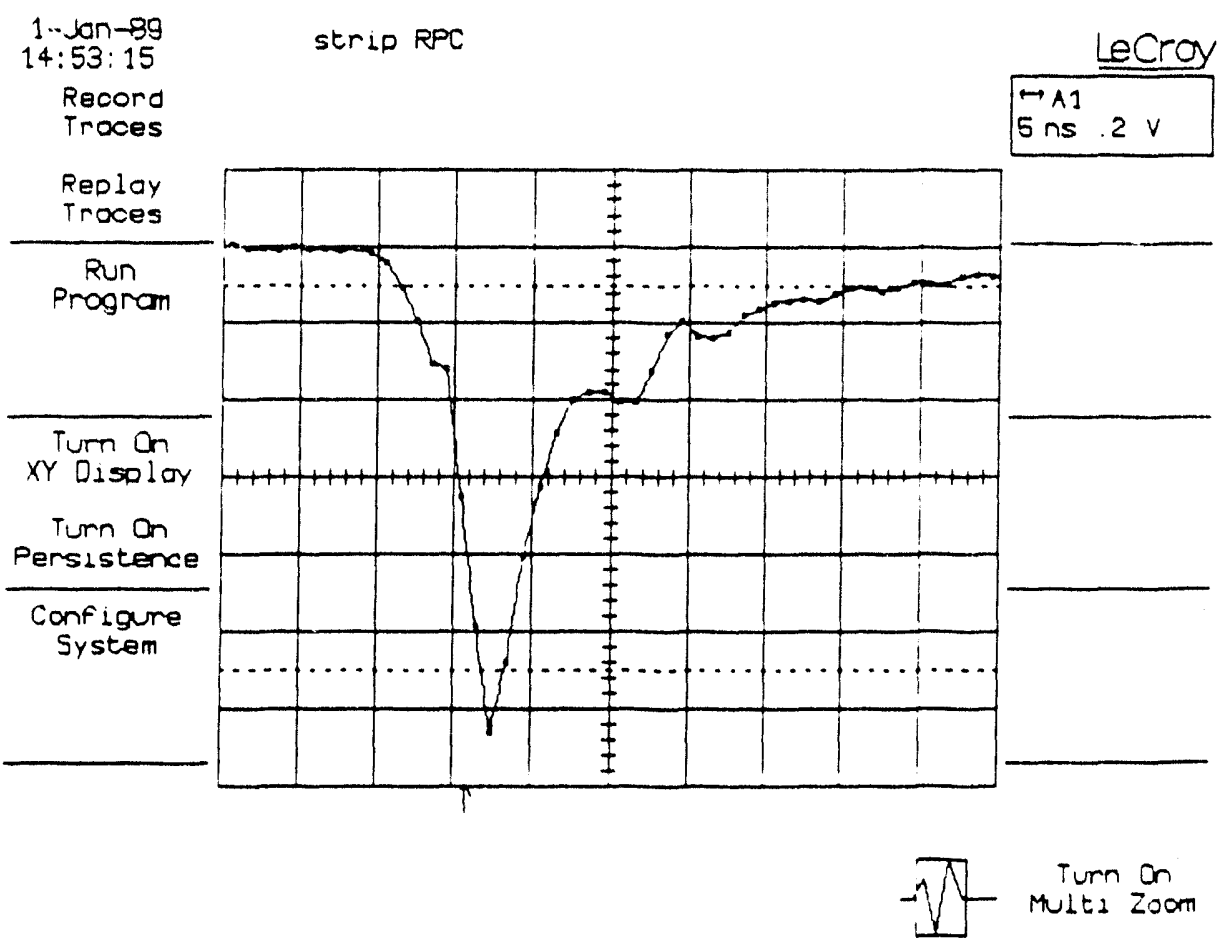

Figure 1 


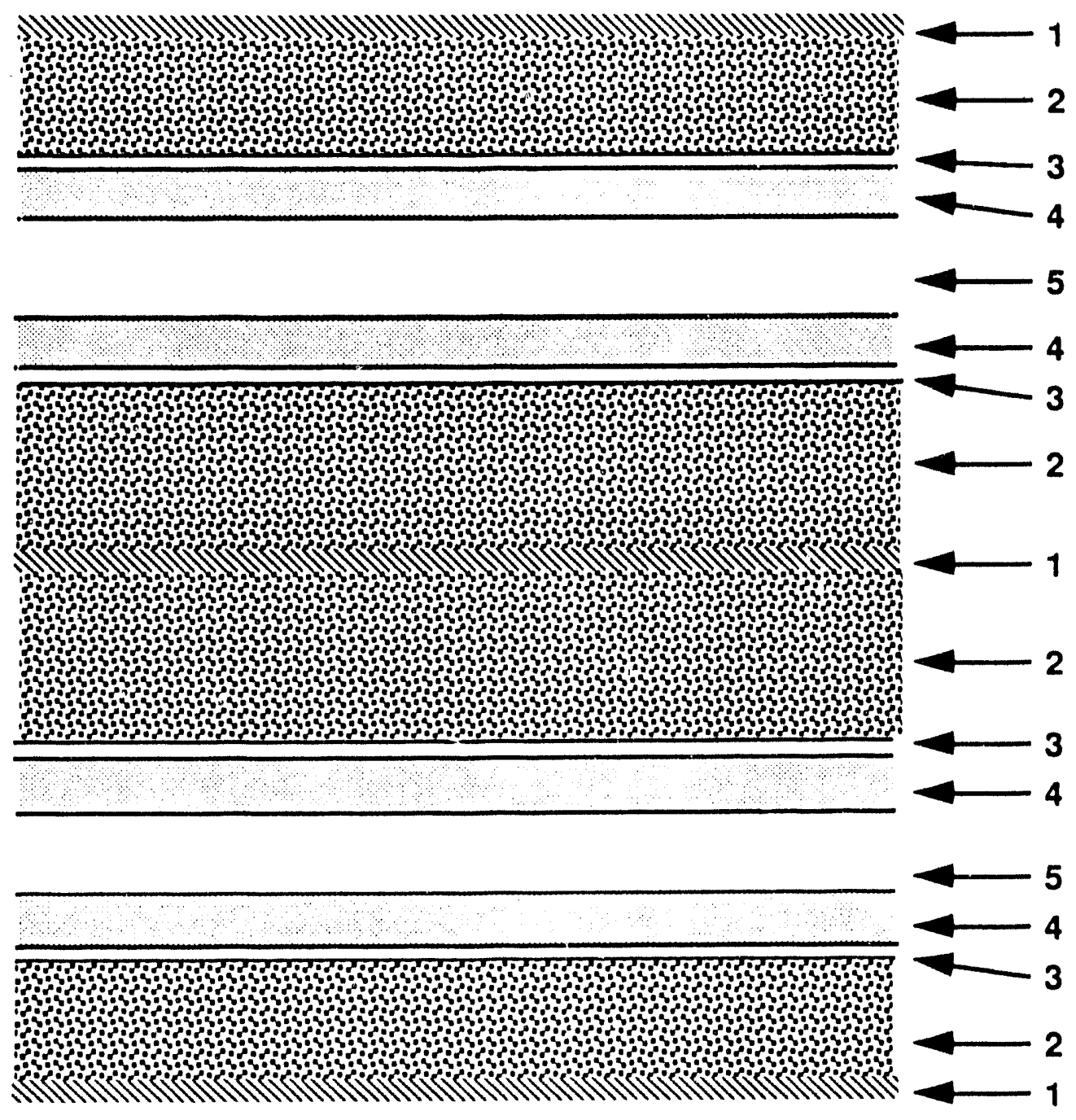

1. 0.010" aluminum

2. $4 \mathrm{~mm}$ foam

3. $0.005^{\prime \prime}$ al. mylar

4. 0.015 " Bakelite(aquadag coating)

5. $2 \mathrm{~mm}$ gas gap

scale 10:1

Figure 2 


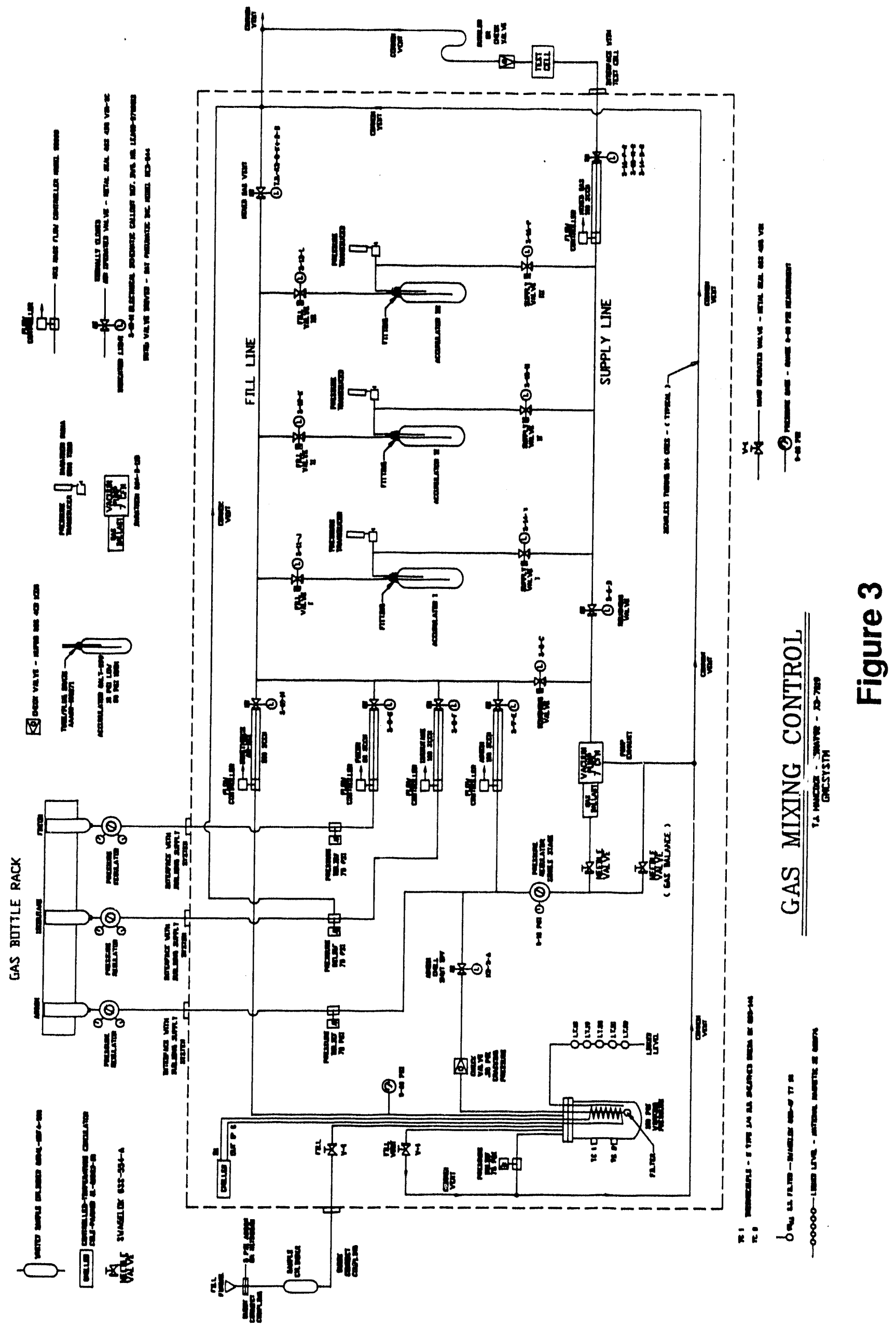




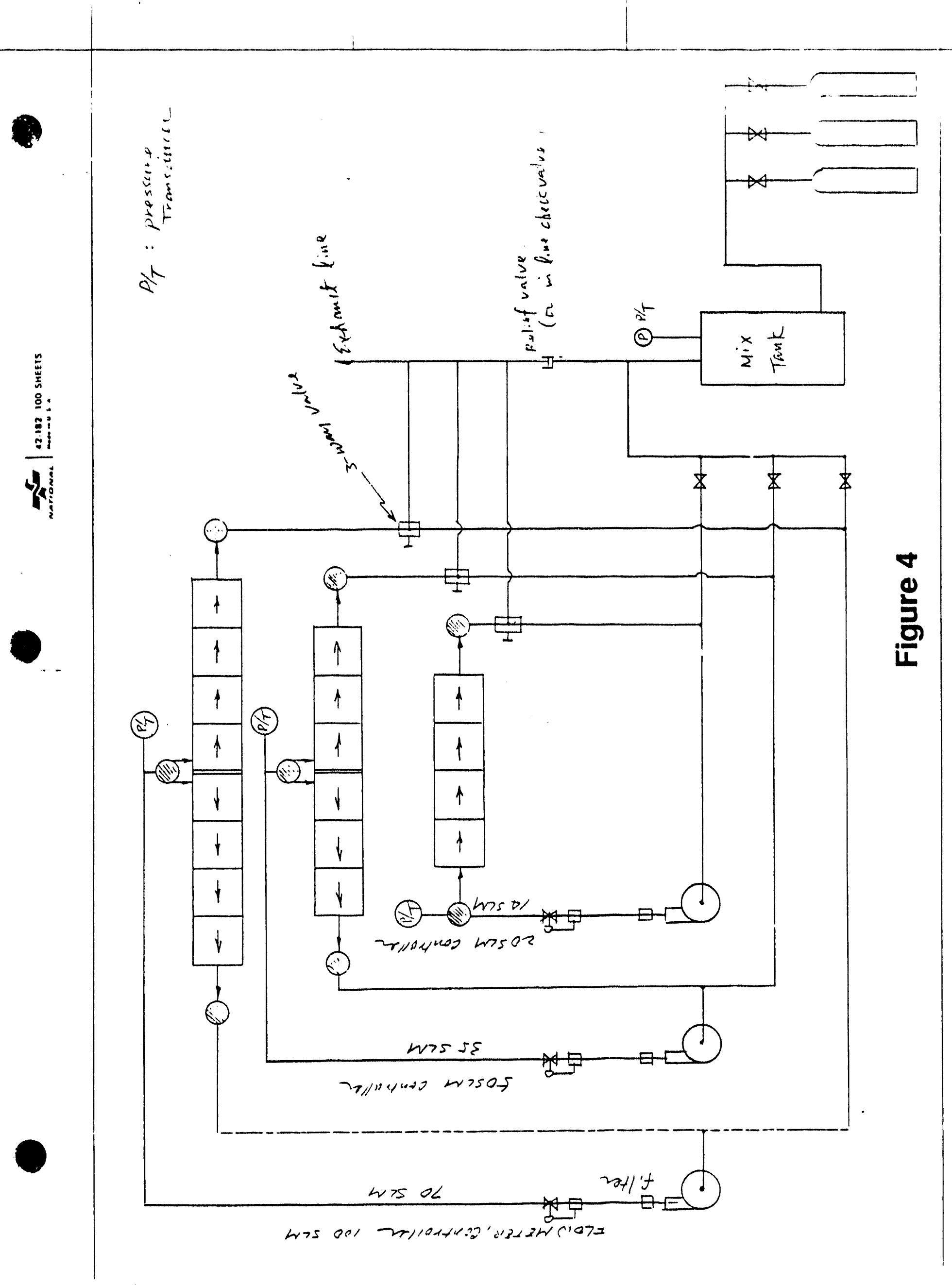




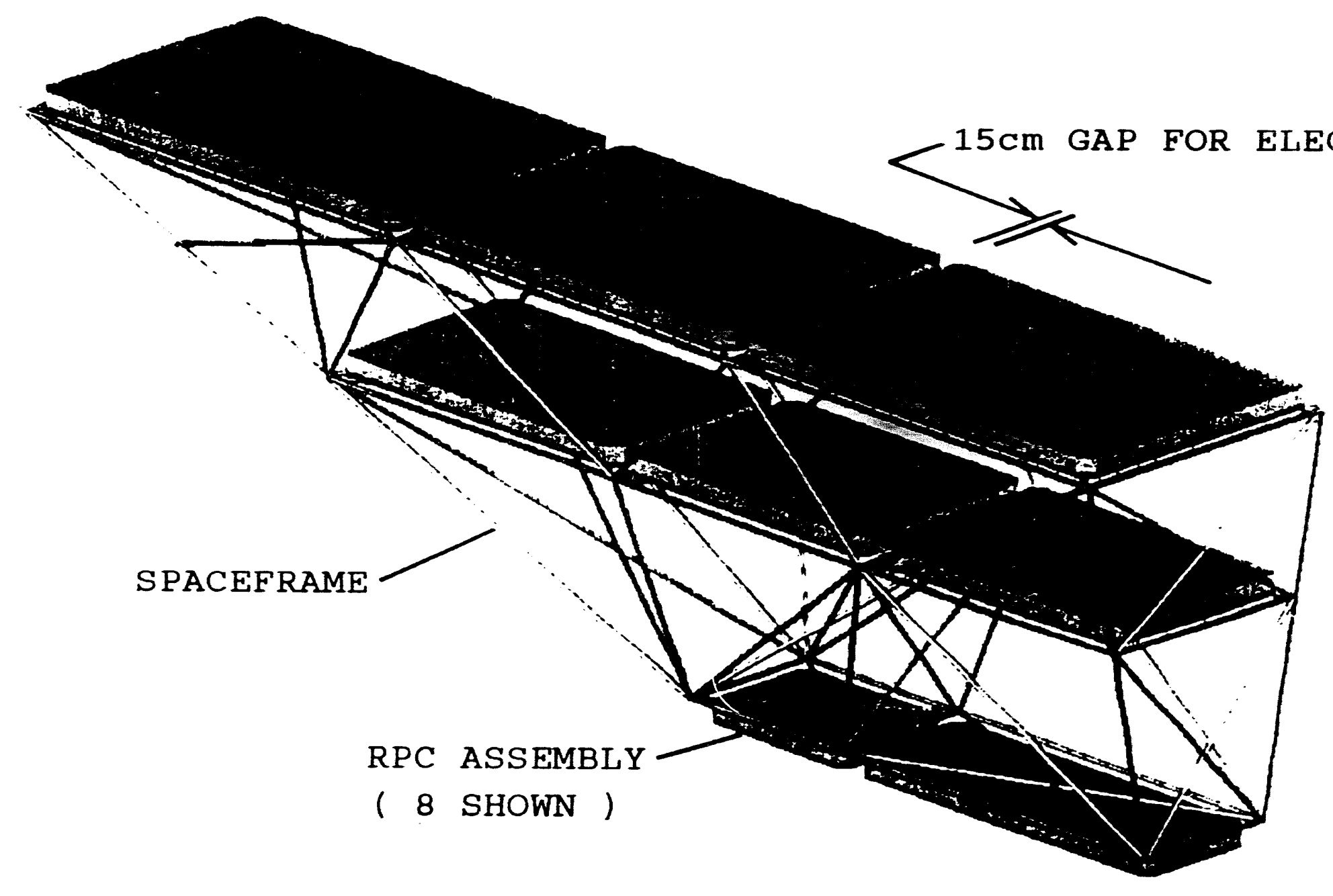

\section{MUON SEGMENT}

Figure 5 


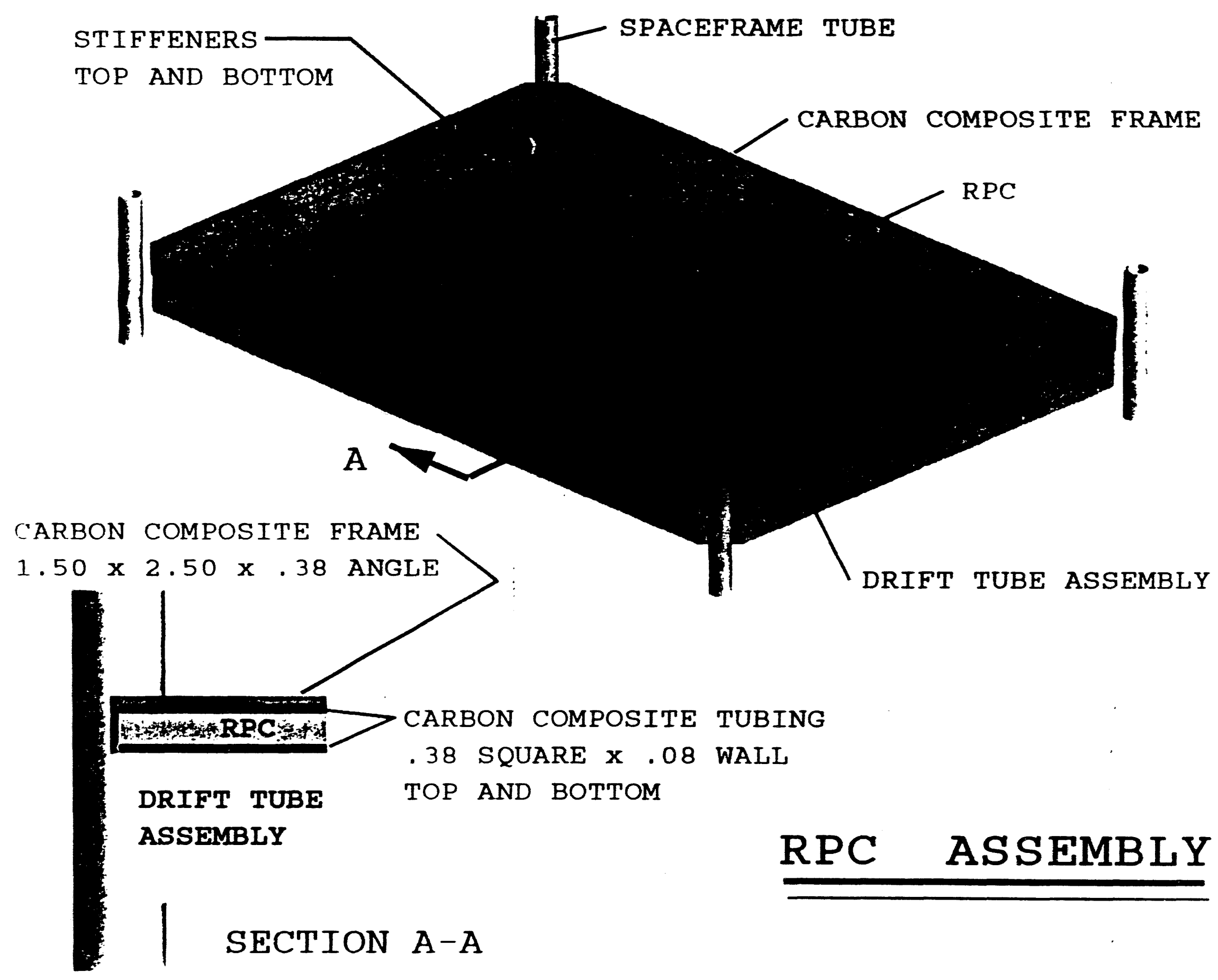

Figure 6 
Inner RPC box layout - 2 chambers overlap per sector

$\times 16$ sectors $\times 2$ halves $=64$ chambers

- $28.1 \mathrm{~cm}$ overlap
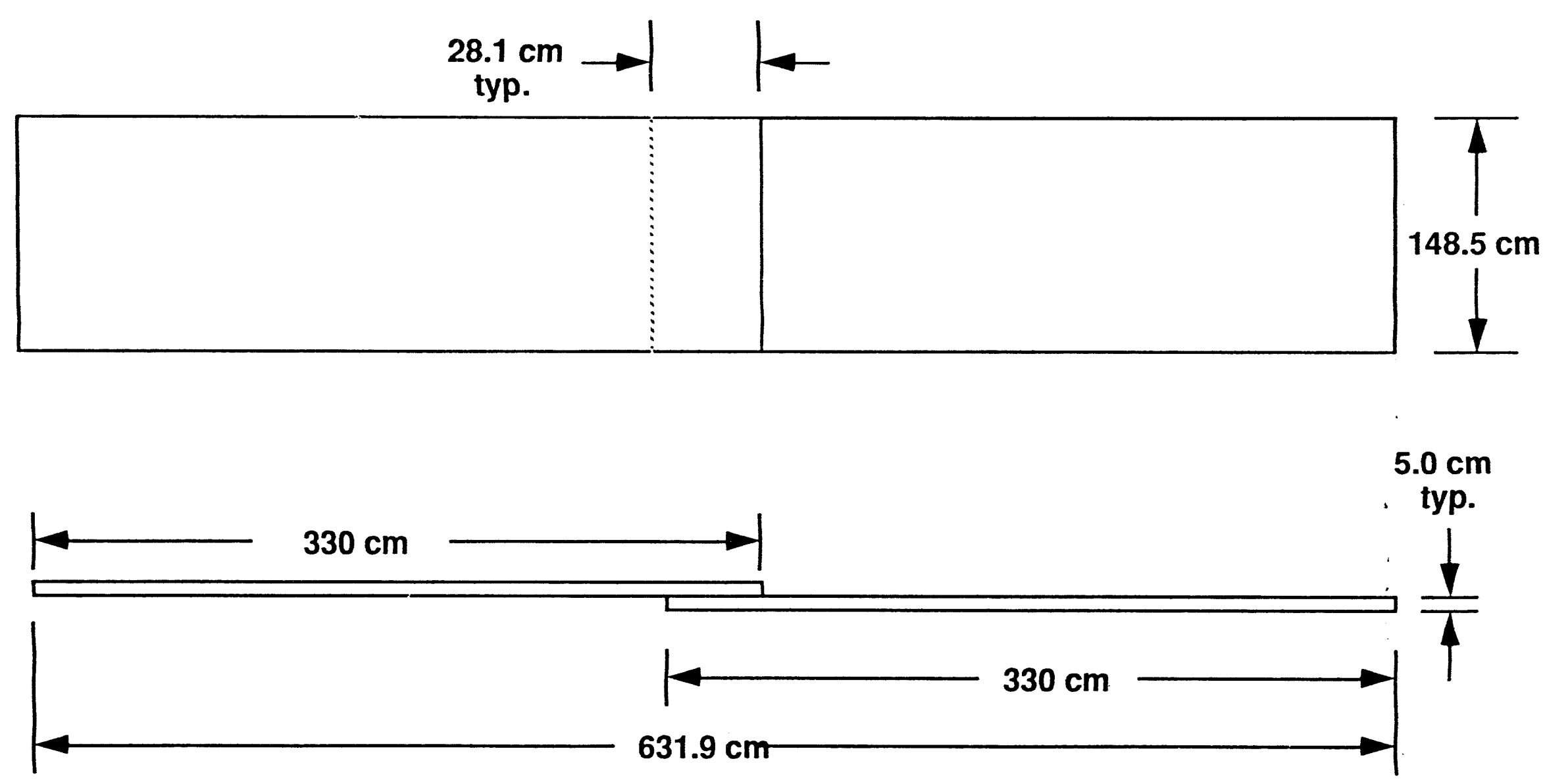

Figure 7 
Middle RPC box layout - 3 chambers overlap per sector $\times 16$ sectors $\times 2$ halves $=96$ chambers

- 18.7 cm overlap

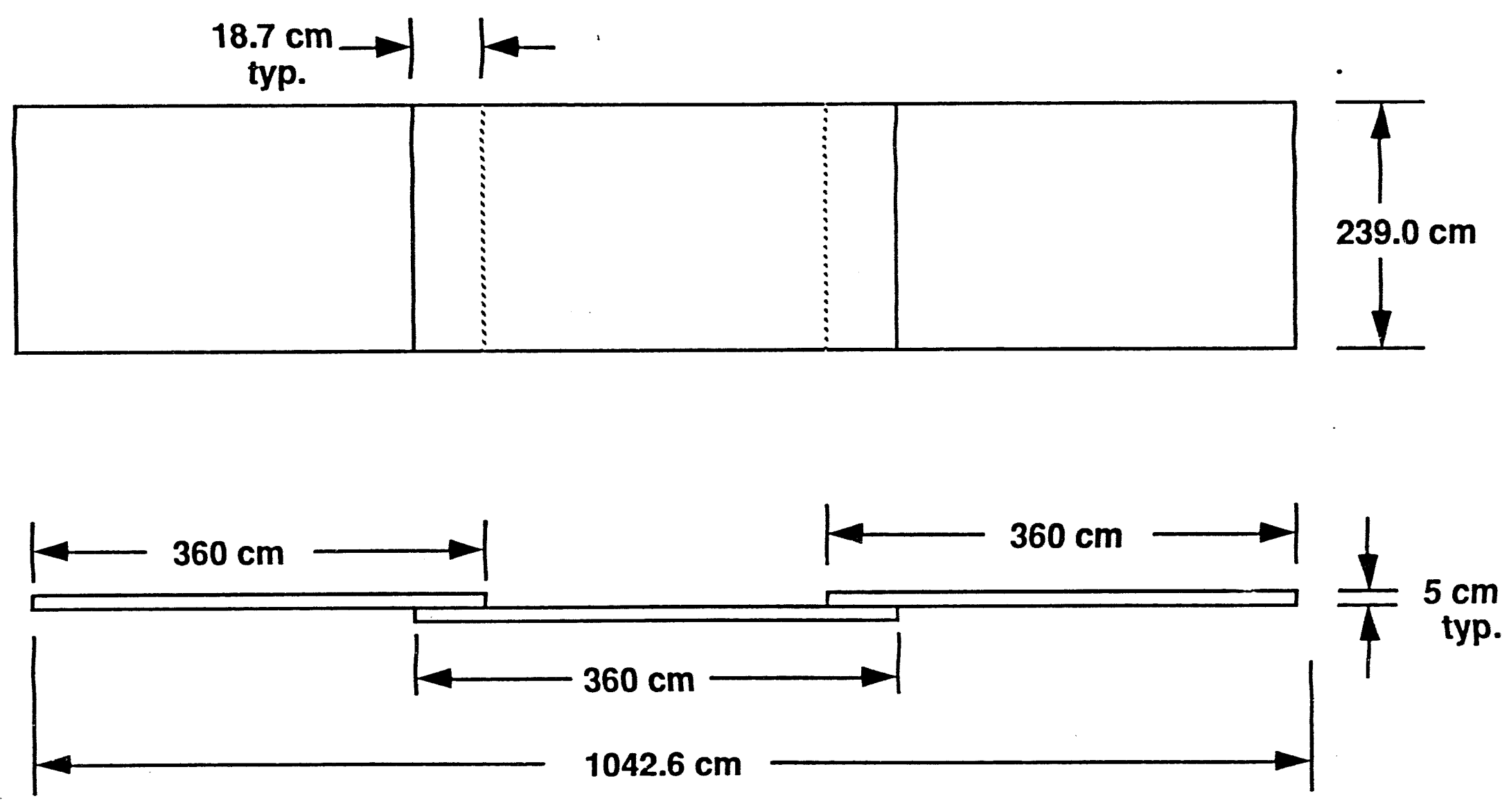

Figure 8 
Outer RPC box layout - 4 chambers overlap per sector

$x 16$ sectors $\times 2$ halves $=128$ shambers

- $15.0 \mathrm{~cm}$ overlap
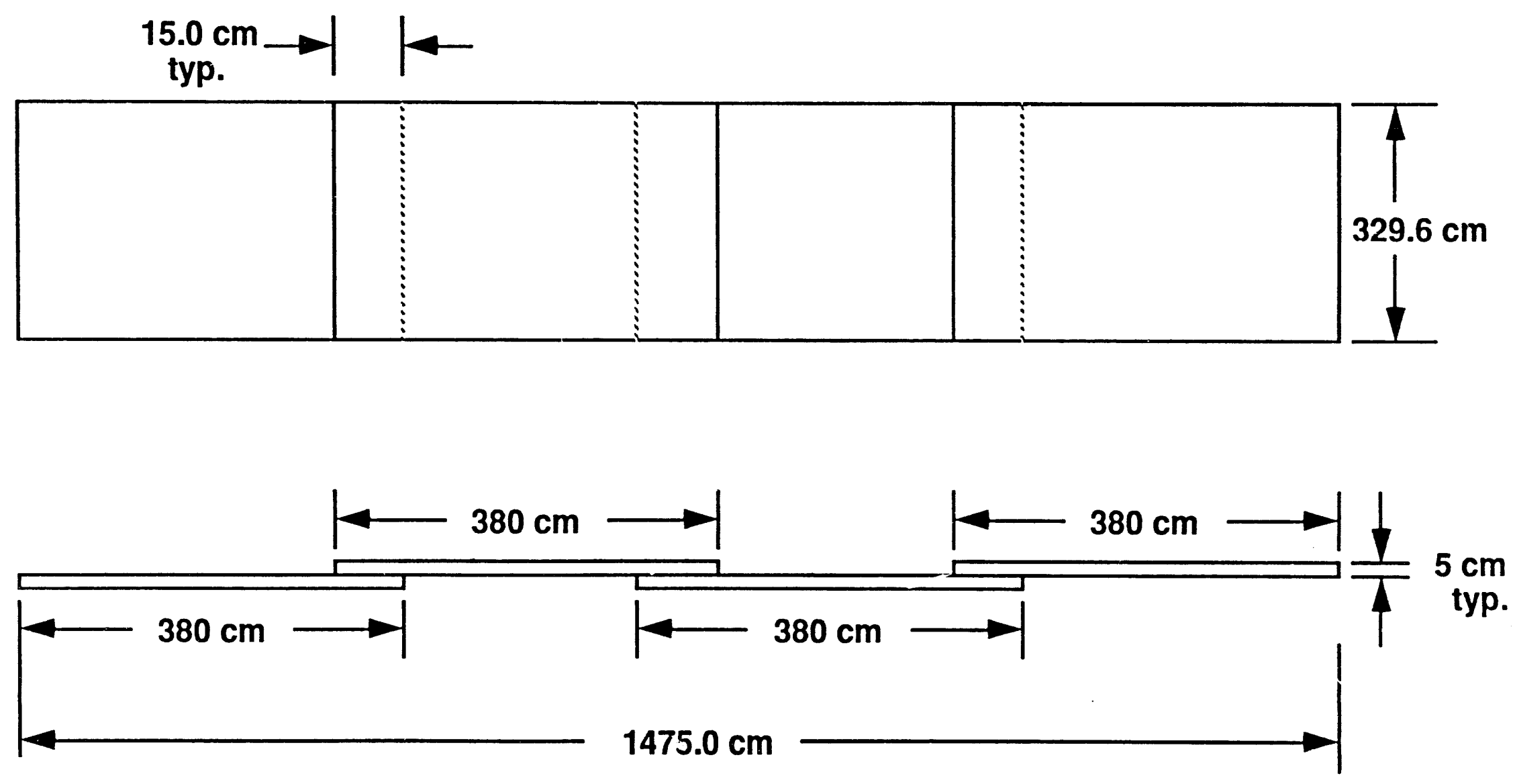

Figure 9 
Foam

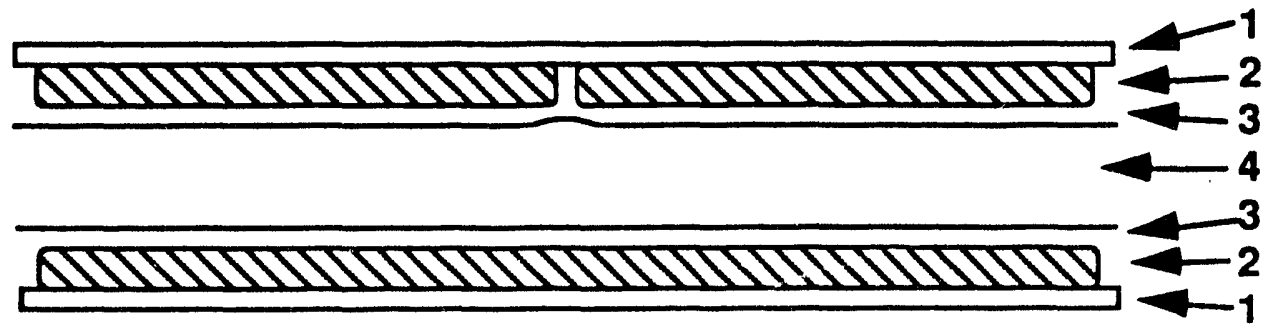

Foam

1. Al Mylar

2. 0.015" Bakelite

3. Linseed oil coating

4. $2 \mathrm{~mm}$ gas gap

Figure 10 


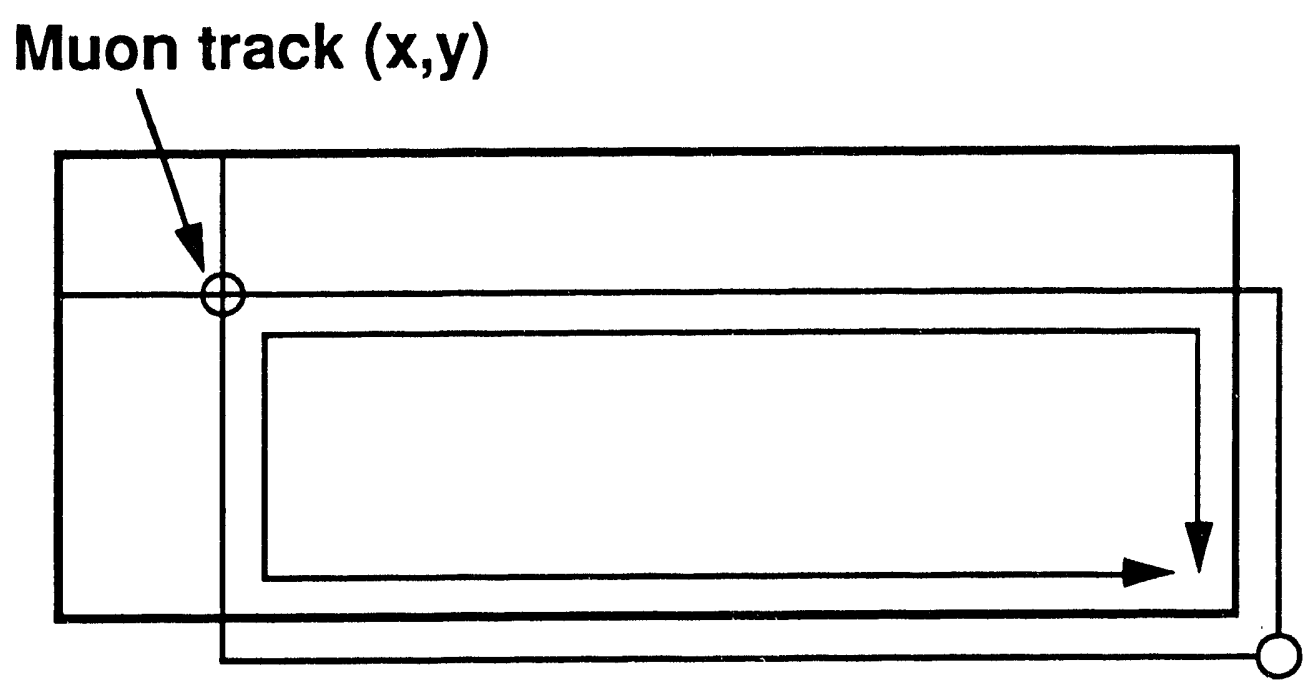

(a)

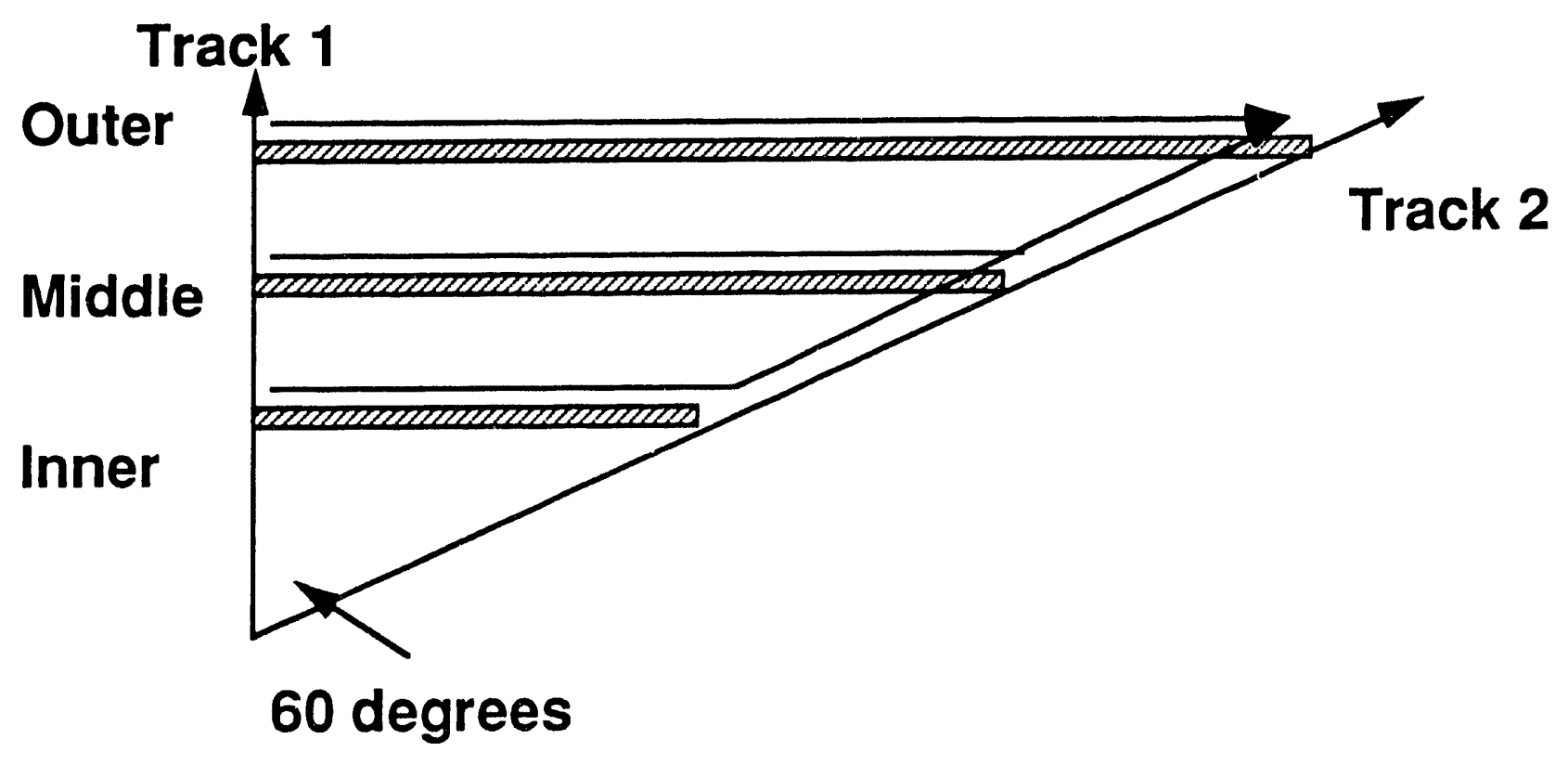

(b)

Figure 11 

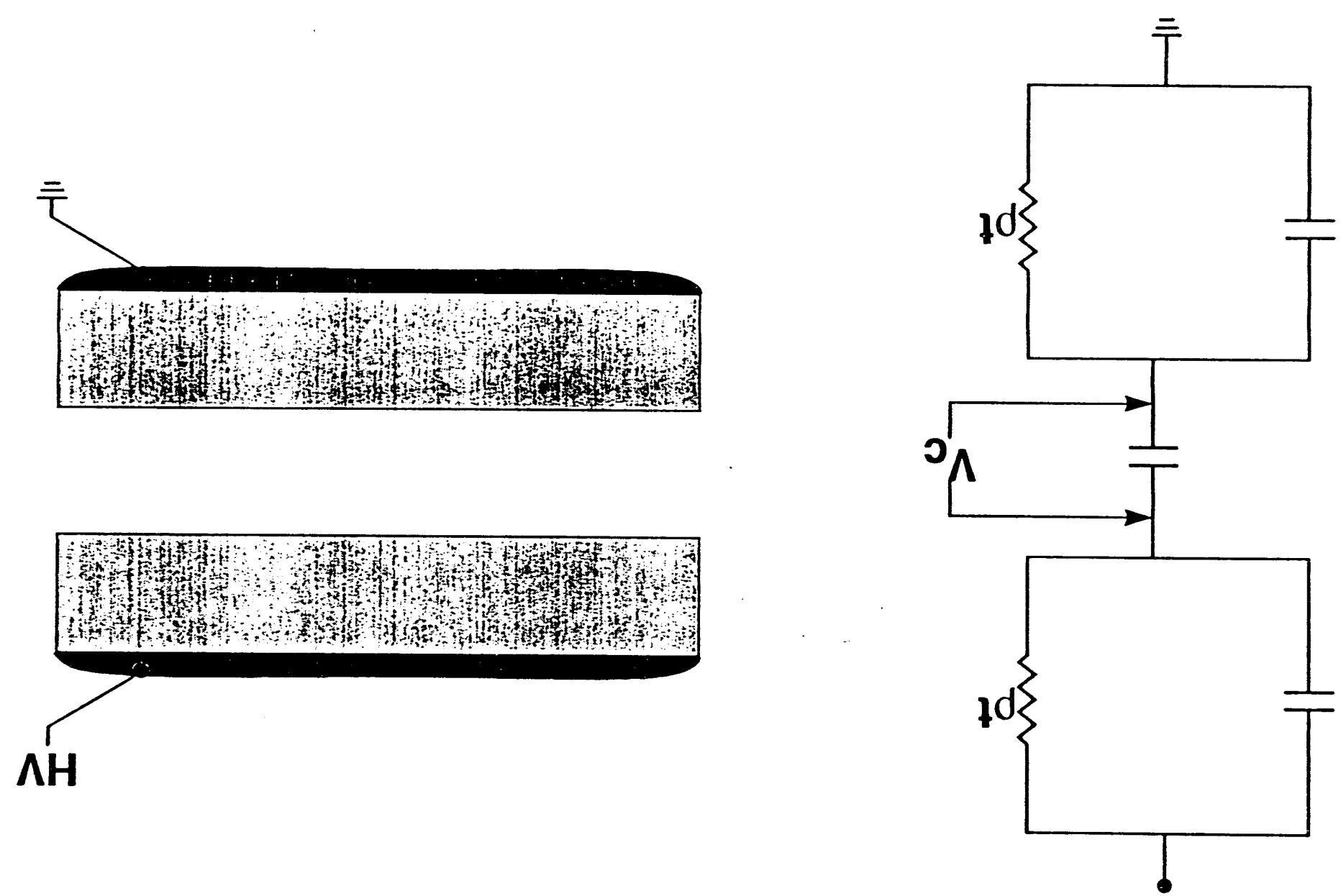


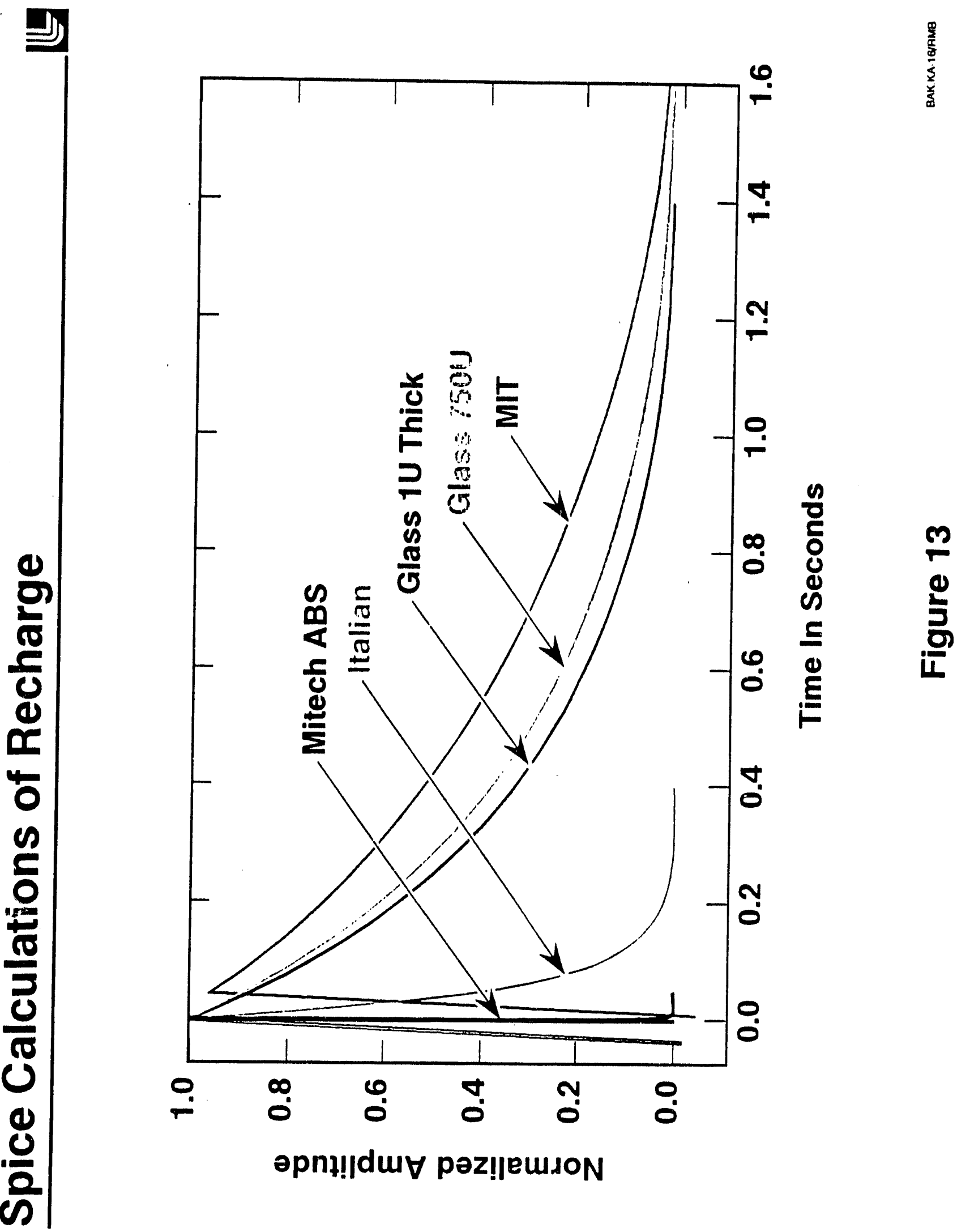




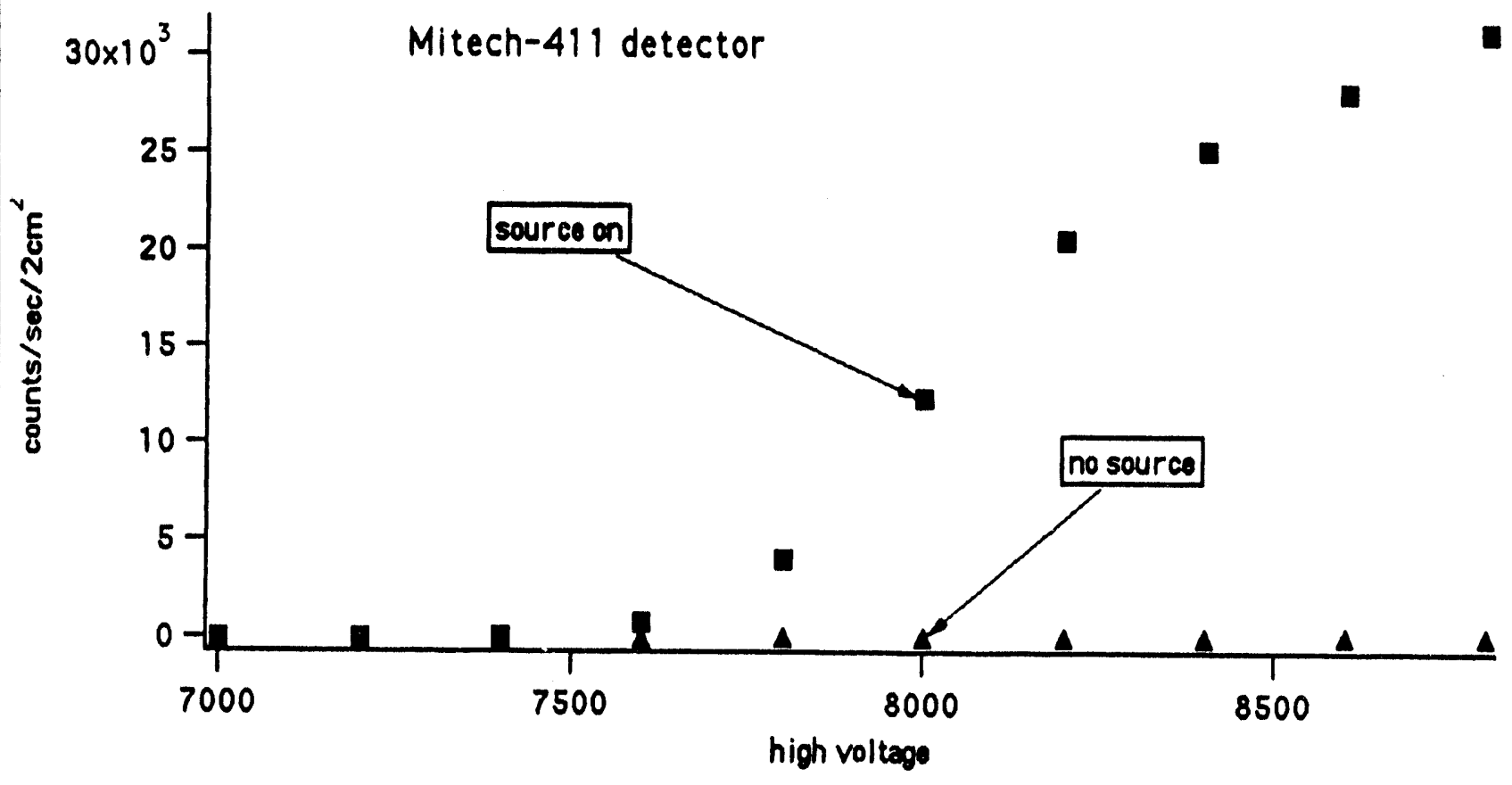

Figure 14 
Sat singles vs spice $1 /$ tau

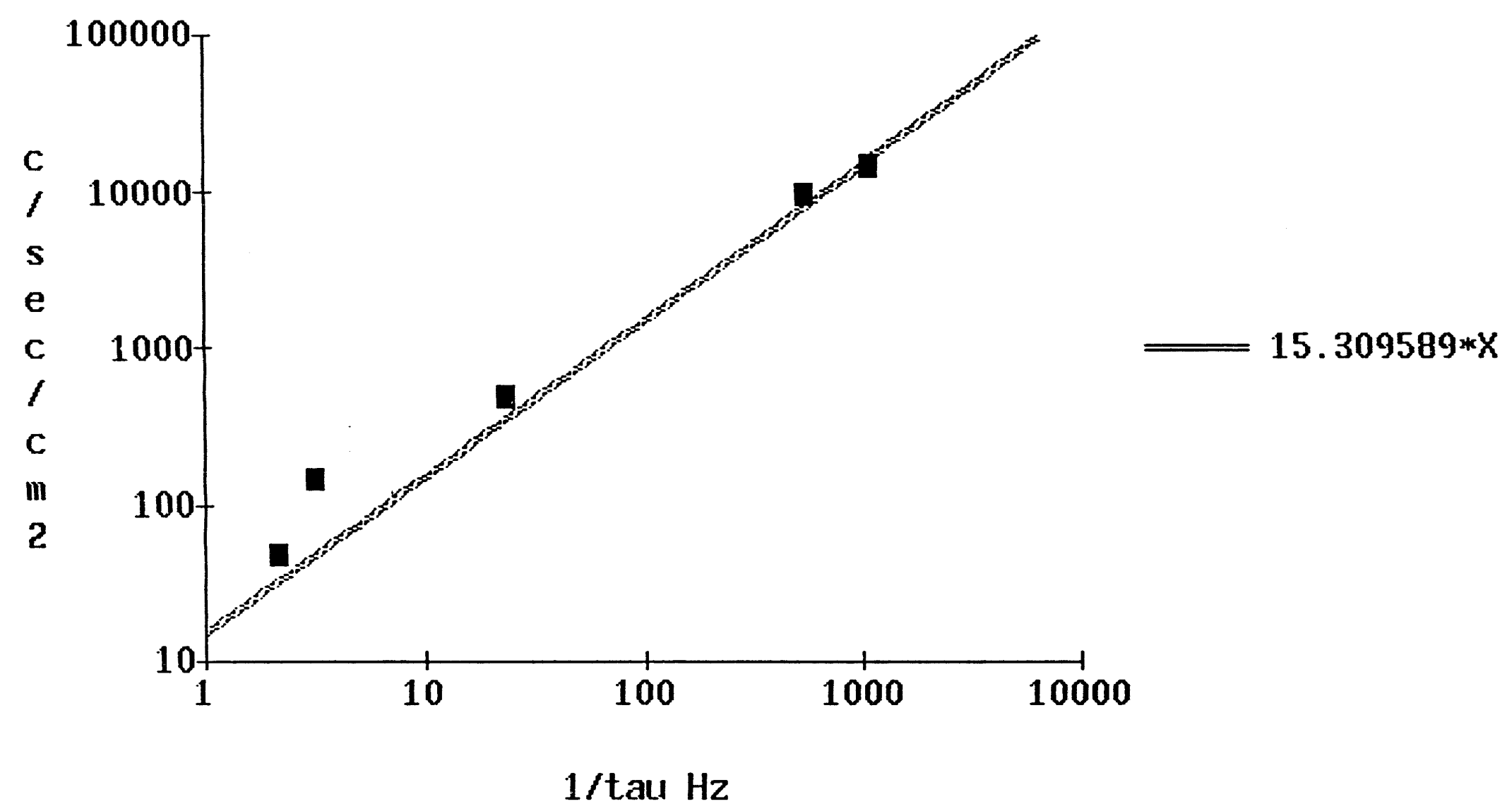

Figure 15 


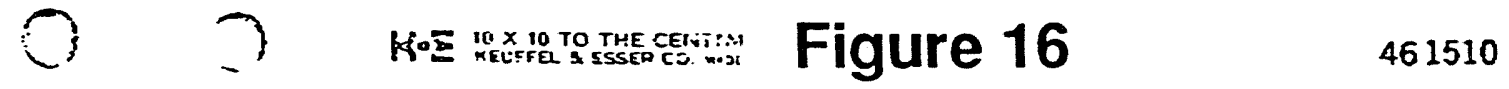

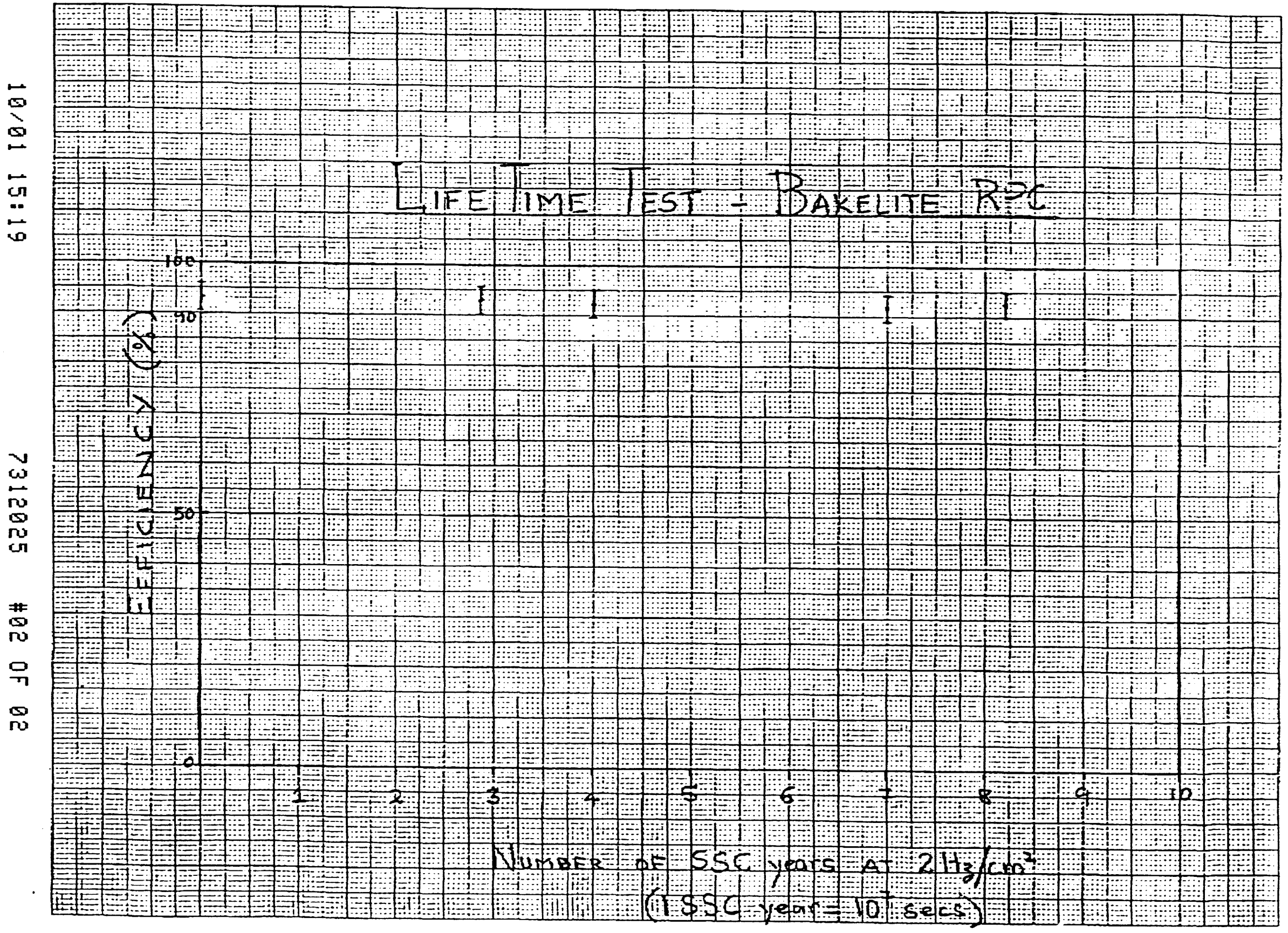




\section{Lᄂ อ.กn6!]}

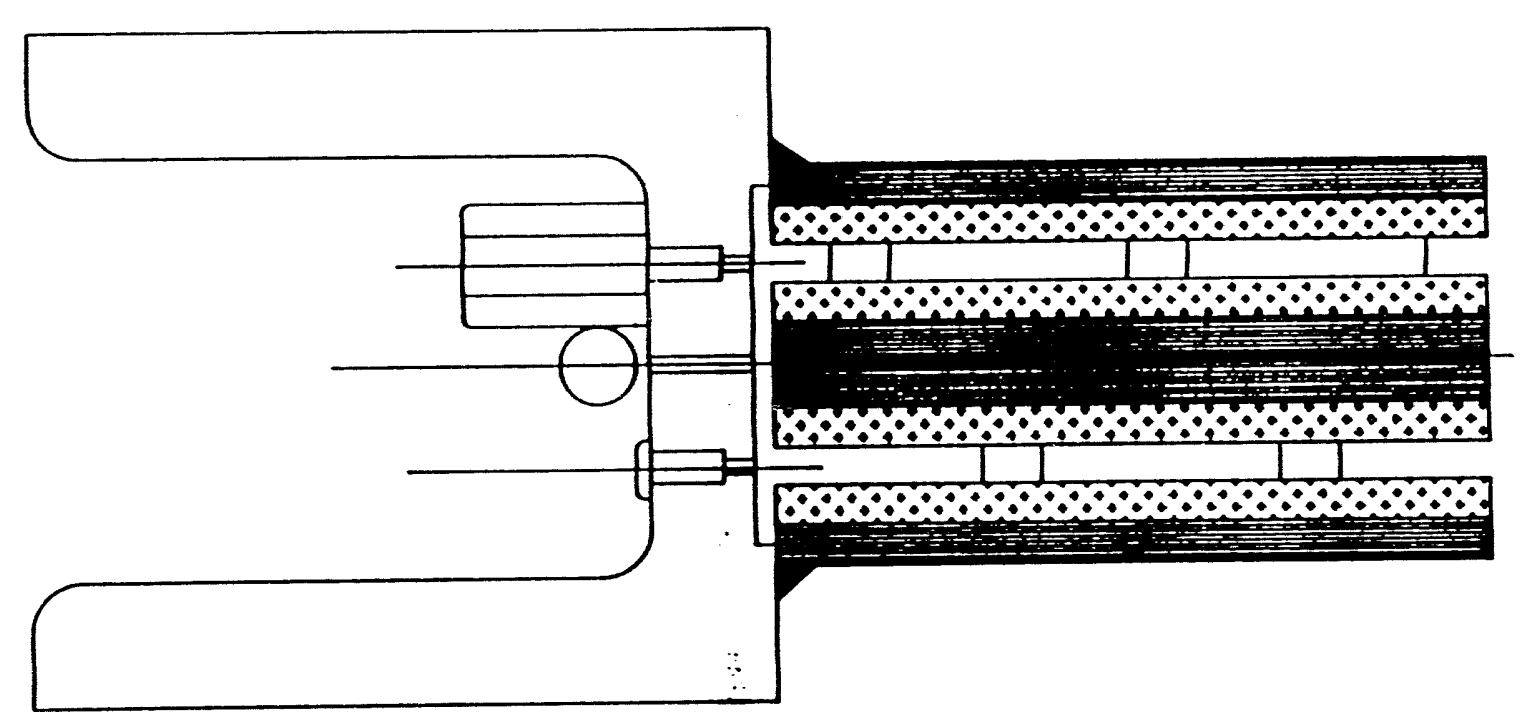




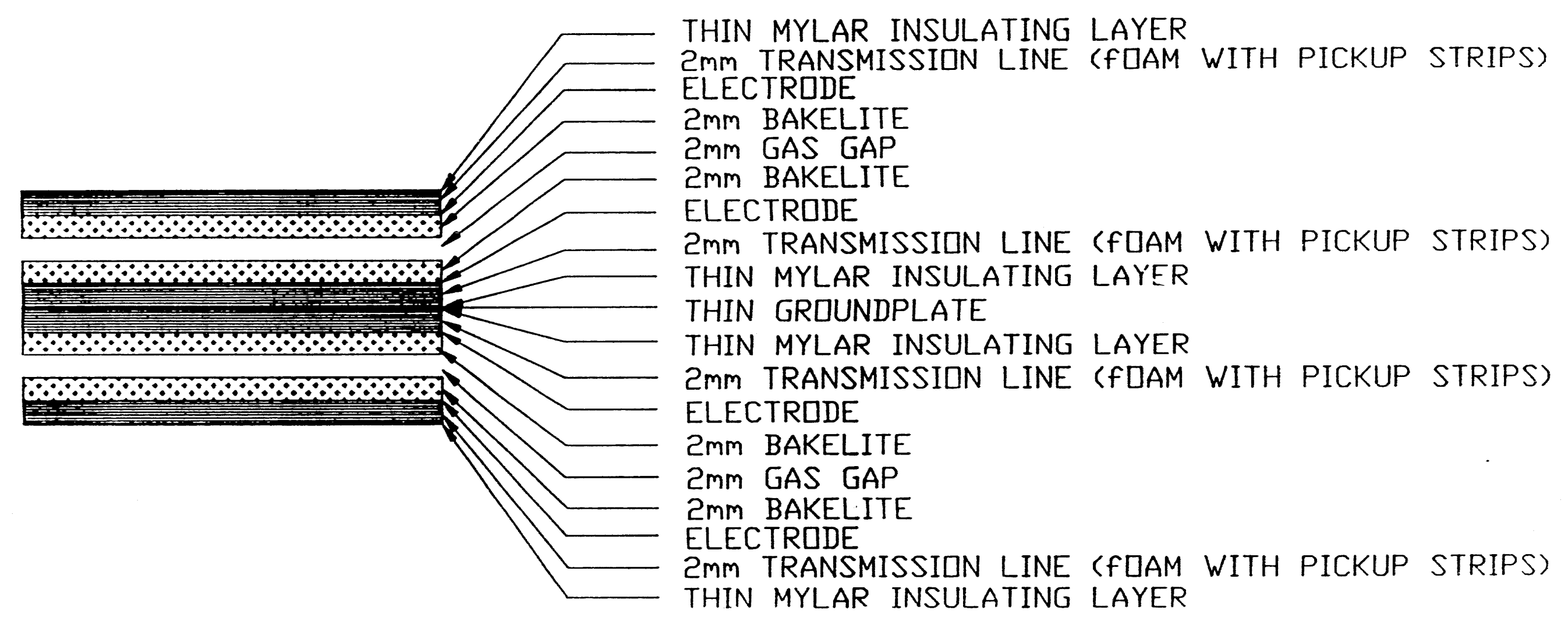

Figure 18 

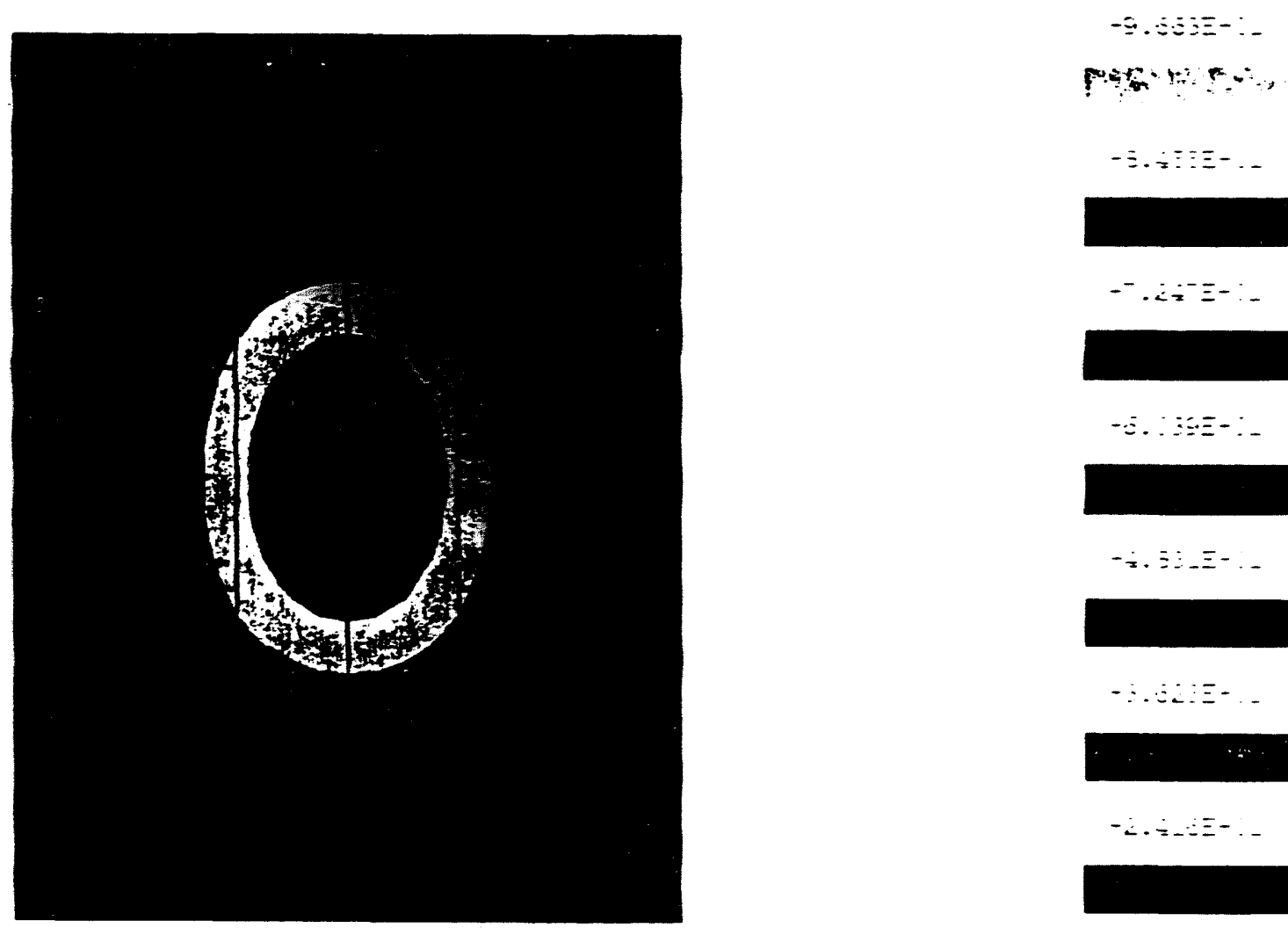

$-^{-} . \therefore \Sigma^{-} \equiv-:-$

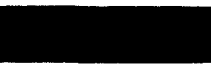

$-\therefore$.

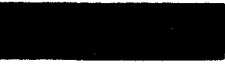

- -

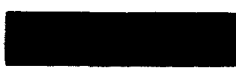

$-\therefore \therefore:$

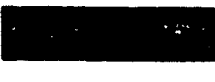

$-2 \therefore-\therefore \equiv-$

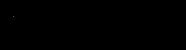

$\because \ldots \equiv \equiv-$

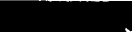

L.

Figure 20 


\author{
APPENDIX 1 \\ Presentation to the Muon Trigger Meeting \\ at the SSC Laboratory \\ I. A. Pless \\ January 22, 1992
}




\section{PRESENTATION TO THE MUON TRIGGER MEETING AT THE SSC LABORATORY 22 JANUARY 1992 \\ IRWIN A. PLESS}

BARREL RPC TRIGGER

I. INTRODUCTION

II. RATES

III. TIMING AND TRIGGER WIDTHS

IV. TRIGGER STRATEGY

V. HARDWIRE IMPLEMENTATION

VI. ASSOCIATIVE MEMORY IMPLEMENTATION

VII. SUMMARY 
I. INTRODUCTION

THIS PRESENTATION IS DESIGNED TO DISCUSS ONLY THE TOPIC OF THE BARREL RPC TRIGGER. SOME ASPECTS OF THE TOPIC CONSIDERED MIGHT HAVE RELEVANCE TO THE FORWARD DIRECTION.

WE HAVE AS OUR STARTING DATA THE CURVES CALCULATED BY B. ZHOU AND R. MCNEIL. THESE SEEM TO BE IN AGREEMENT WITH THE TABLE CALCULATED BY D. GREEN AND D. HEDIN.

WE WILL SHOW THAT TAKING ADVANTAGE OF THE ISOCHRONISITY OF THE BARREL GEOMETRY, OUR TRIGGER WIDTH NEED ONLY BE NINE NANO-SECONDS WIDE, PLUS THE RISE TIME JITTER OF THE RPC. WE SHOW THAT AT THE LEVEL 2 TRIGGER, THE BEAM CROSSING TIME IS KNOWN TO THE RISE TIME JITTER OF THE RPC. THE BEAM CROSSING TIME AT THE LEVEL 1 TRIGGER SHOULD BE KNOWN TO BETTER THAN 12 NANO-SECONDS. THIS MEANS THAT AT THE LEVEL 1 TRIGGER THE BEAM CROSSING IS UNAMBIGUOUSLY KNOWN. THE TRUTH OF THIS STATEMENT DEPENDS ON THE RPC RISE TIME JITTER BEING LESS THAN 4 NANO-SECONDS. THIS IS ONE OF MANY RPC PROPERTIES THAT WILL BE MEASURED BY THE RPC R AND D PROGRAM.

WE WILL PROPOSE A FOUR LEVEL TRIGGER. WE WILL ALSO DEMONSTRATE THAT THIS TRIGGER CAN 


\title{
BE IMPLEMENTED IN A HARD WIRE FORM AND
} PROBABLY BE IMPLEMENTED USING ASSOCIATIVE MEMORIES.

\author{
IN OUR SUMMARY WE COLLECT ALL THE \\ FEATURES OF THE TRIGGER SCHEME.
}



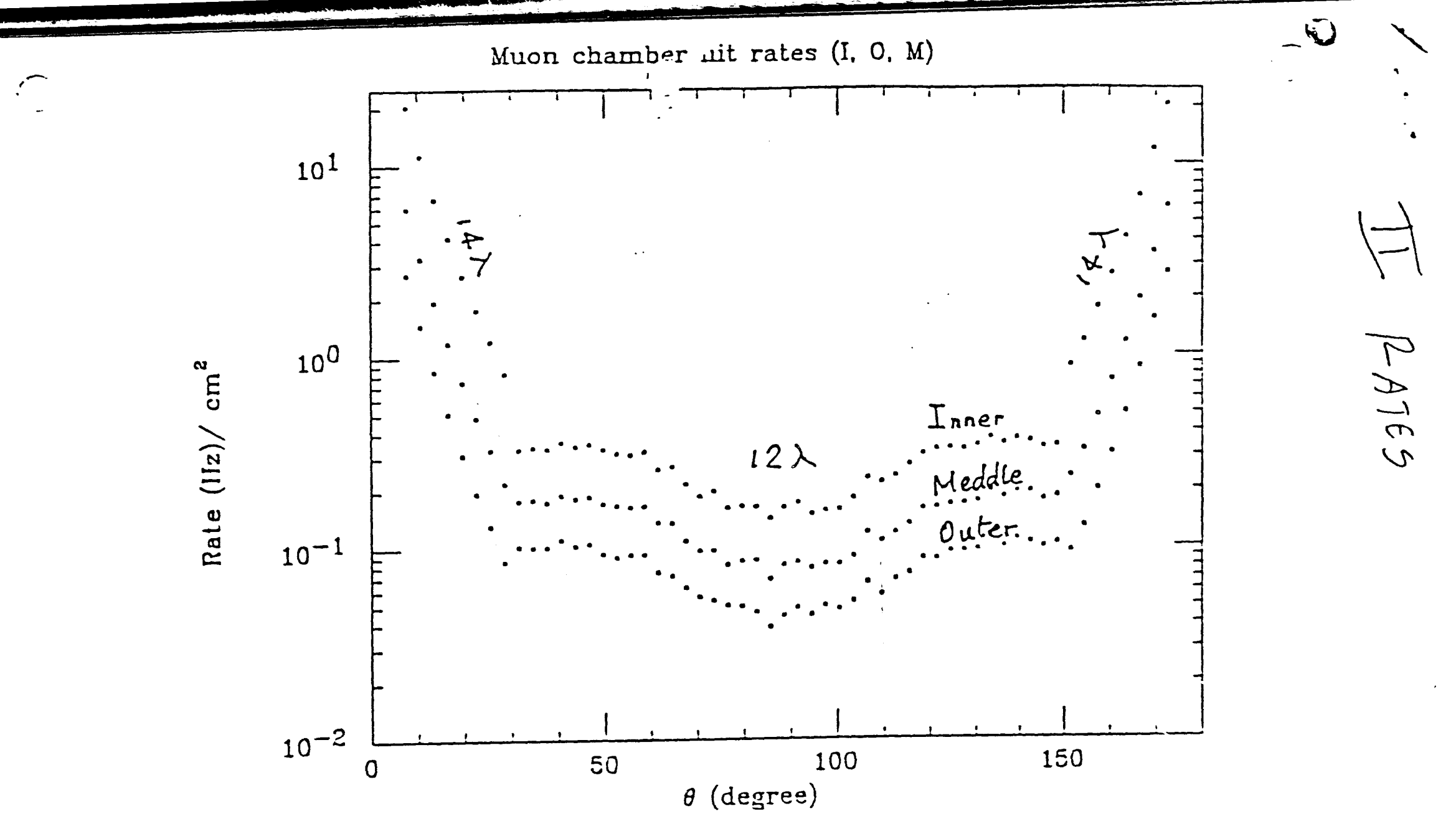

$p$
$\frac{N}{n}$
$v$

Figure 5

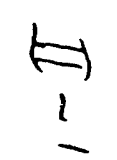



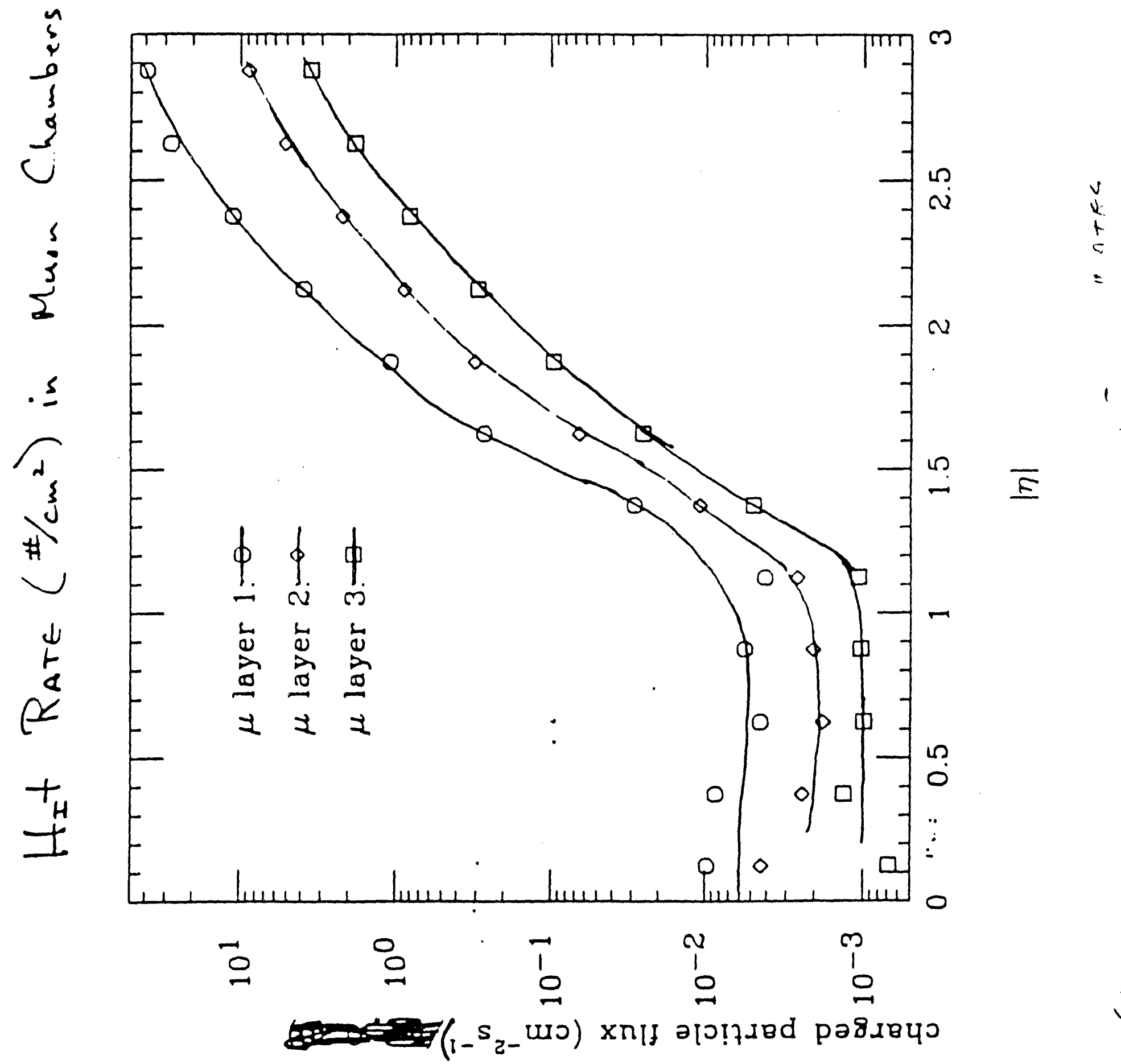


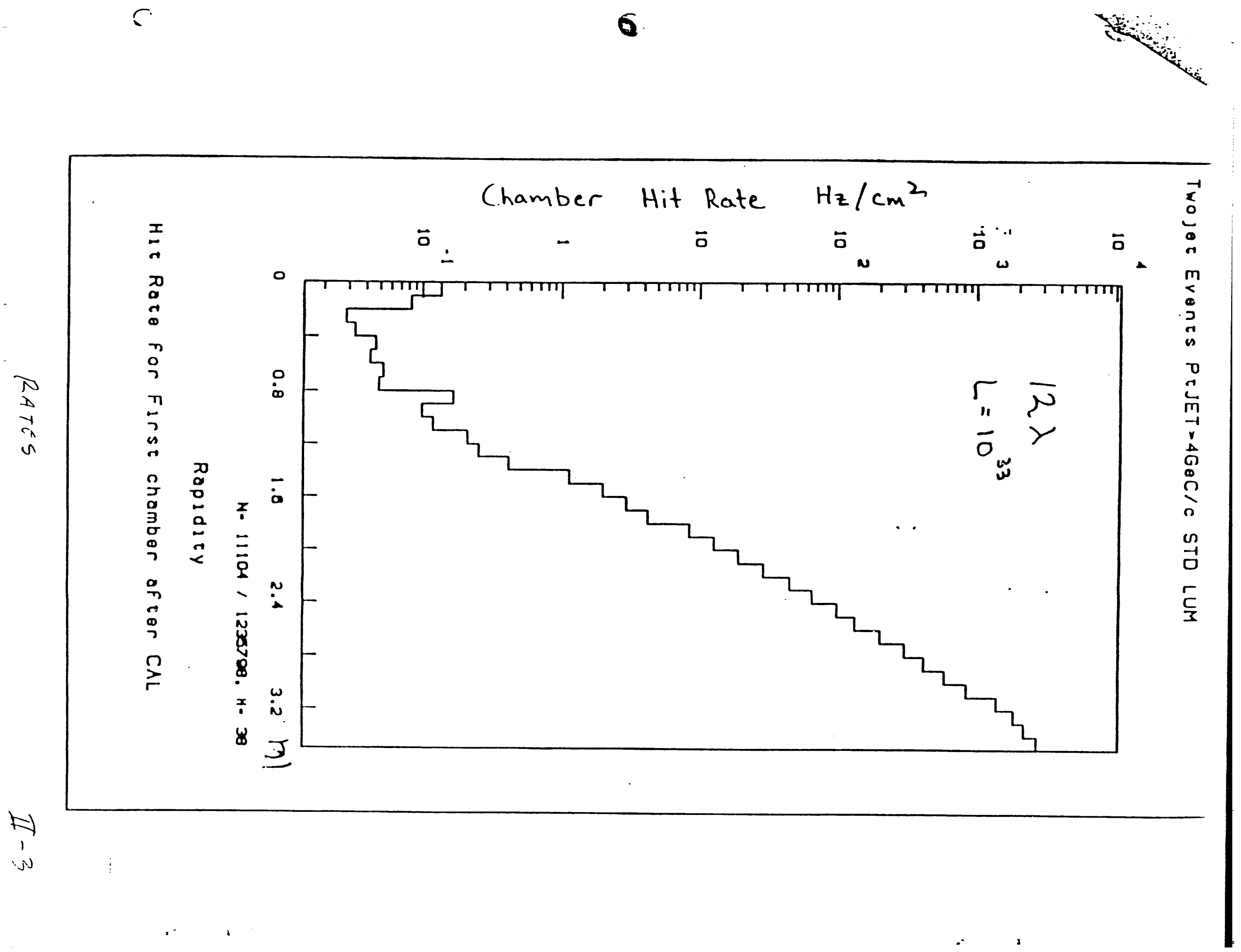




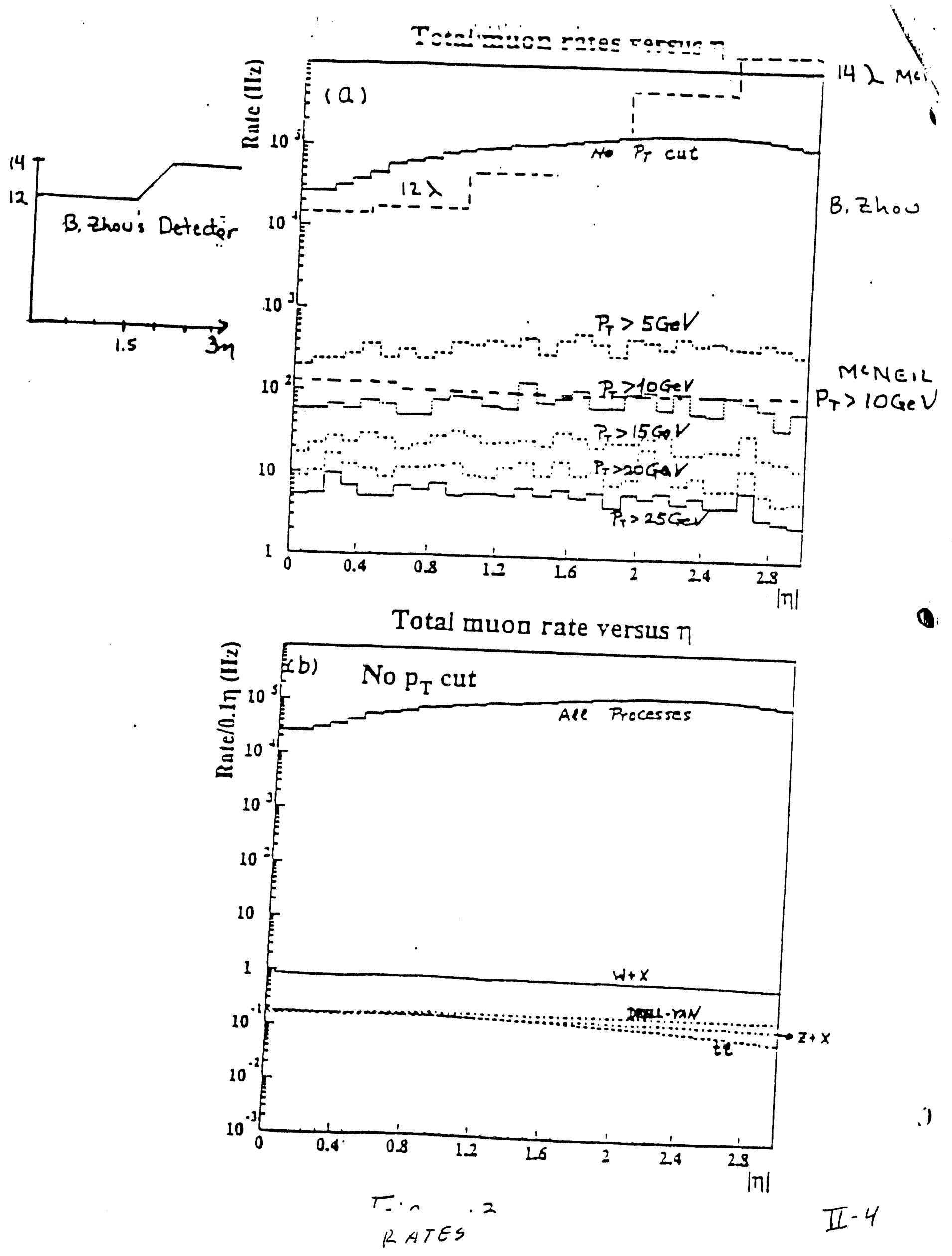


Dan Green $\xi_{1}^{\prime}$ Dave Hedin

Table 2: Muon Rates versus Absorber Thickness

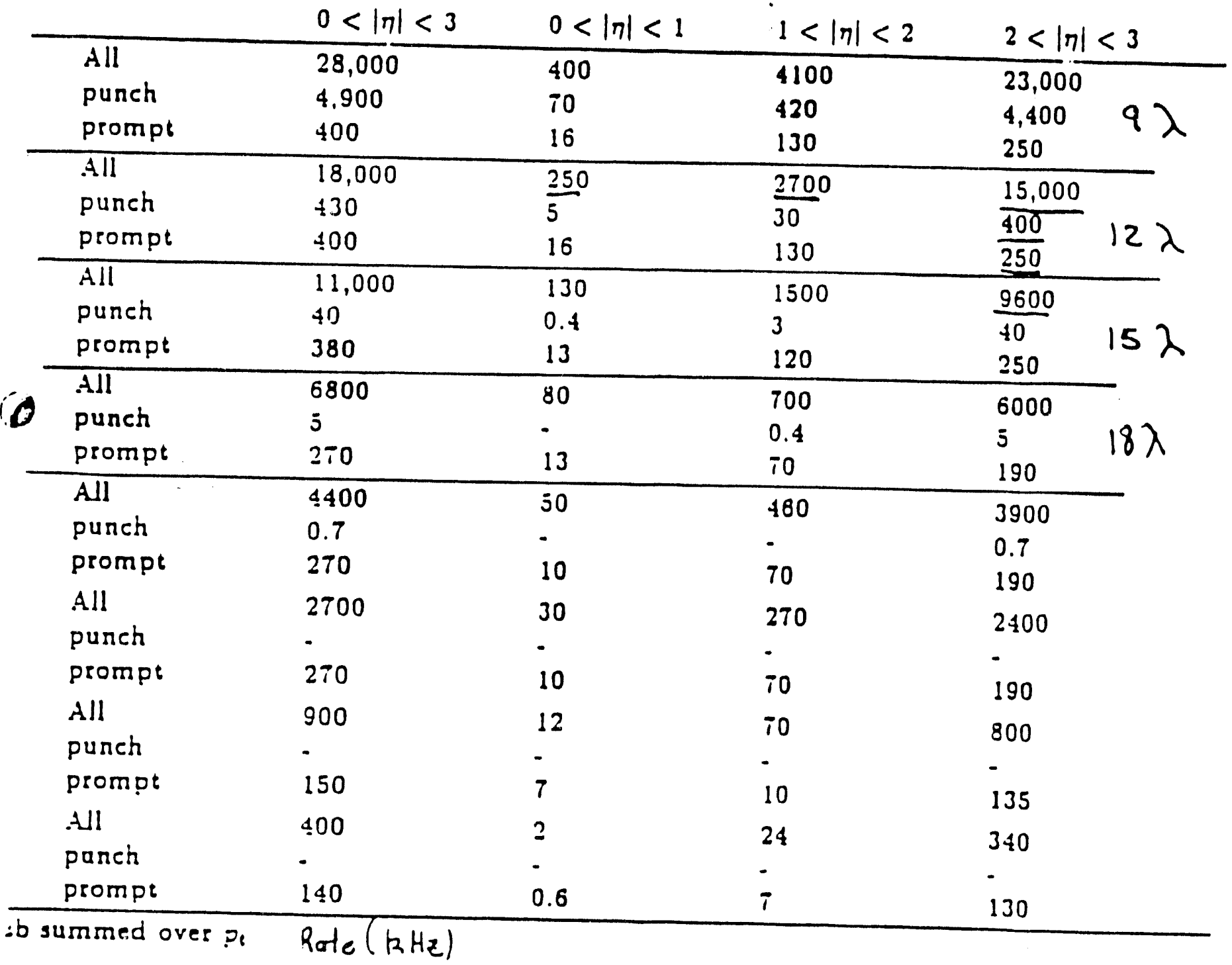


Level 2 ( $P_{\text {TCur }}$ ) 
II. RATES

THE CALCULATIONS I HAVE JUST SHOWN ASSUMES A LUMINOSITY OF $10^{33}$. FOR THIS SAME LUMINOSITY, I INTERPELLATE AND EXTRAPOLATE THE DATA FOR THE BARREL, AND ARRIVE AT THE FOLLOWING RATES FOR THE BARREL. WHERE THE DATA DID NOT AGREE, I ASSUMED THE HIGHER NUMBERS.

TOTAL RATE, NO $P_{t}$ CUT $1.3 \times 10^{6} \mathrm{HERTZ}$

$P_{t}>10 \mathrm{GeV} / \mathrm{c}$

2,000 HERTZ

$P_{t}>20 \mathrm{GeV} / \mathrm{c}$ 200 HERTZ

$P_{t}>30 \mathrm{GeV} / \mathrm{c}$

75 HERTZ

THE TRIGGER LOI OVERVIEW CALCULATED THE FOLLOWING RATES:
$P_{t}>10 \mathrm{GeV} / \mathrm{c}$
1300 HERTZ
$P_{t}>20 \mathrm{GeV} / \mathrm{c}$
390 HERTZ
$P_{t}>30 \mathrm{GeV} / \mathrm{c}$
70 HERTZ

I CONSIDER THIS SURPRISING AGREEMENT.

THEREFORE WE WILL TAKE AS THE RATES THE HIGHER NUMBERS: 


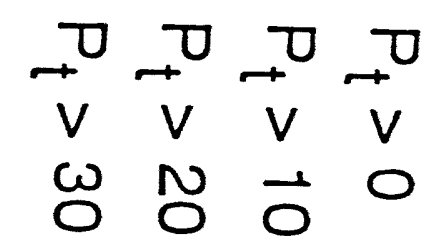

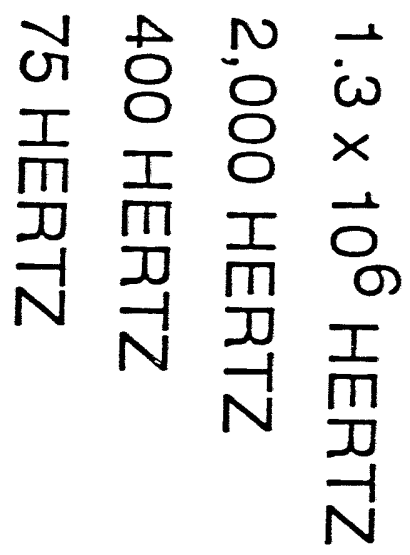


III. TIMING AND TRIGGER WIDTHS

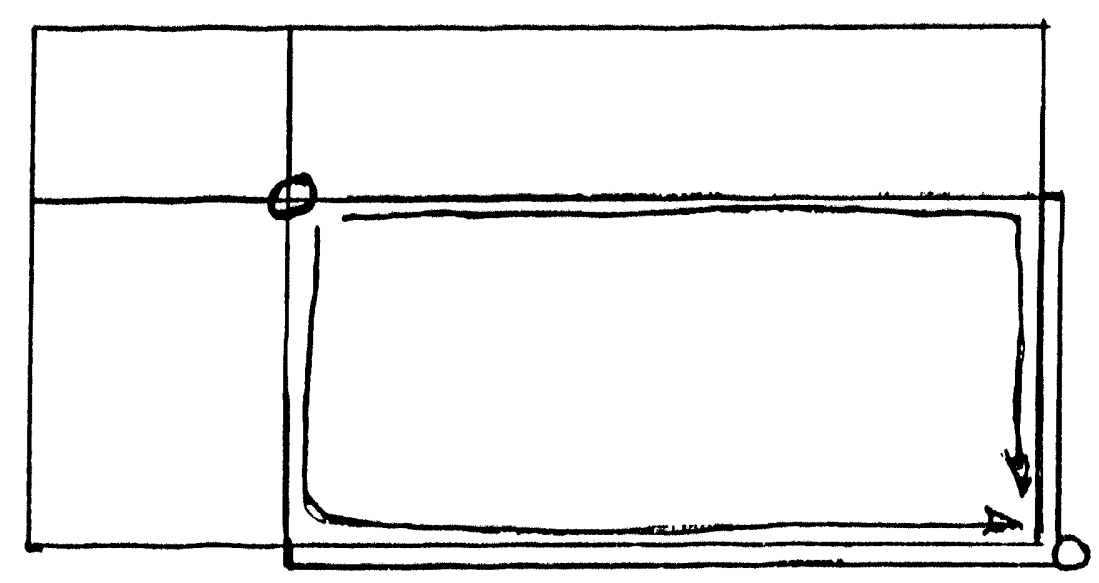

IF WE COLLECT ALL SIGNALS FROM THE STRIPS IN ONE CORNER AND MATCH CABLE SPEEDS TO STRIP SPEEDS WE ARE MATCHED IN TIME AT THE CORNER. AT THE LEVEL 1 TRIGGER WE KNOW THE BEAM CROSSING TO BETTER THAN 12 NANO-SECONDS, 8 NANO-SECONDS DUE TO THE $1.59 \mathrm{M}$ LENGTH OF THE STRIP AND 4 NANO-SECONDS DUE TO THE RISE TIME JITTER OF THE RPC PULSE. THIS LATTER NUMBER HAS TO BE VERIFIED BY THE RPC R AND D.

HOWEVER, AT THE TIME OF THE FIRST LEVEL TRIGGER, THE IDENTITY OF THE X AND Y STRIPS ARE KNOWN. AT THIS POINT, ONE CAN CALCULATE THE BEAM CROSSING TIME TO AN ACCURACY OF THE RISE TIME JITTER OF THE RPC. 


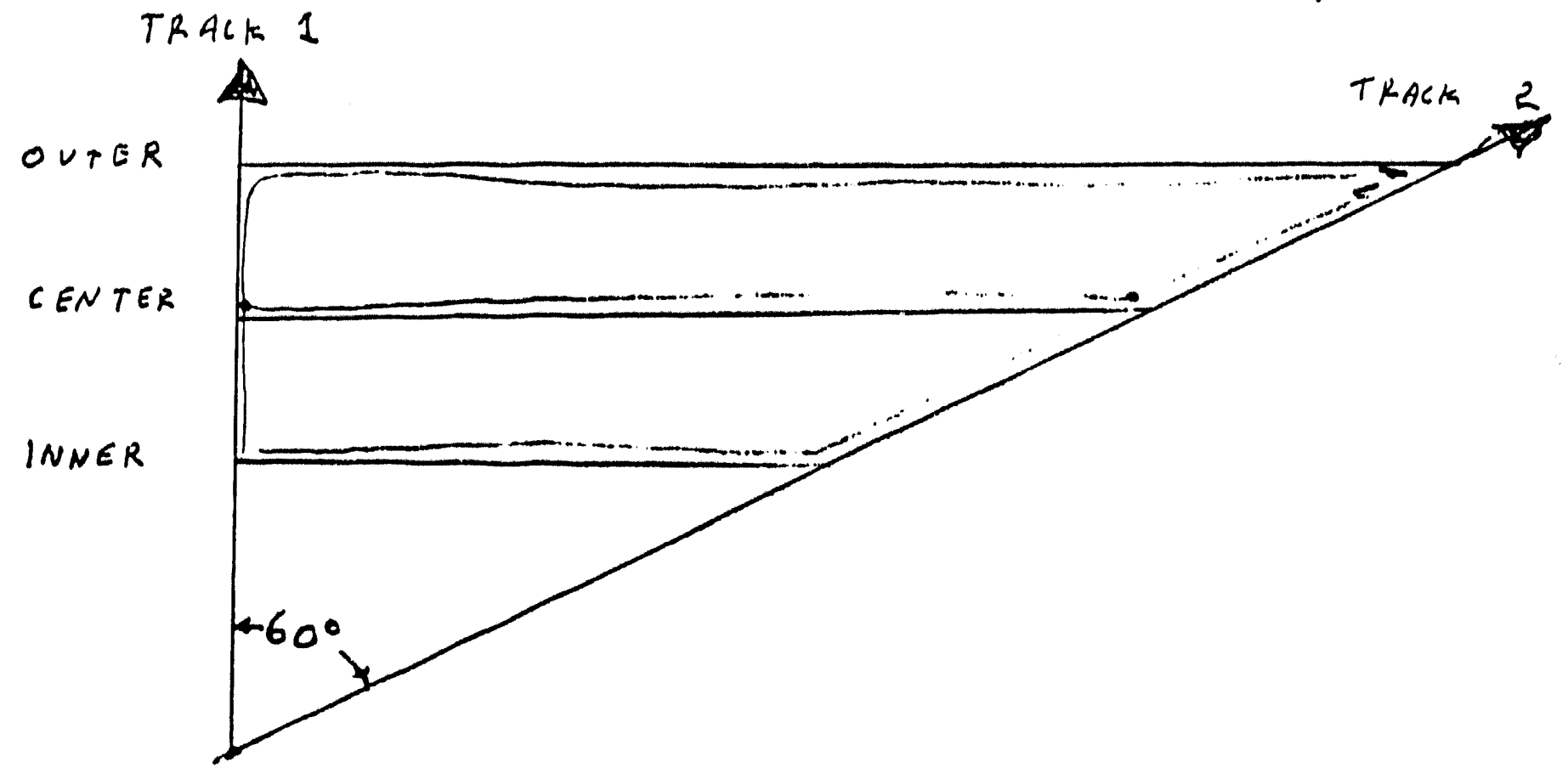

FOR TRACK 1, THE SIGNAL FROM THE OUTER LAYER ARRIVES 9 NANO-SECONDS AFTER THE SIGNAL FROM THE INNER LAYER AND 4.5 NANO-SECONDS AFTER THE SIGNAL FROM THE CENTER LAYER.

FOR TRACK 2, THE SIGNAL FROM THE OUTER LAYER ARRIVES 13 NANO-SECONDS BEFORE THE SIGNAL FROM THE INNER LAYER AND 6.5 NANO-SECONDS BEFORE THE SIGNAL FROM THE CENTER LAYER.

HENCE THE TRIGGER WIDTHS ON THE OUTER LAYER MUST BE 13 NANO-SECONDS LONG, WHILE THE TRIGGER WIDTHS ON THE INNER LAYER MUST BE 9 NANO-SECONDS LONG AND THE TRIGGER WIDTHS OF THE CENTER LAYER MUST BE 6.5 NANO-SECONDS LONG. 


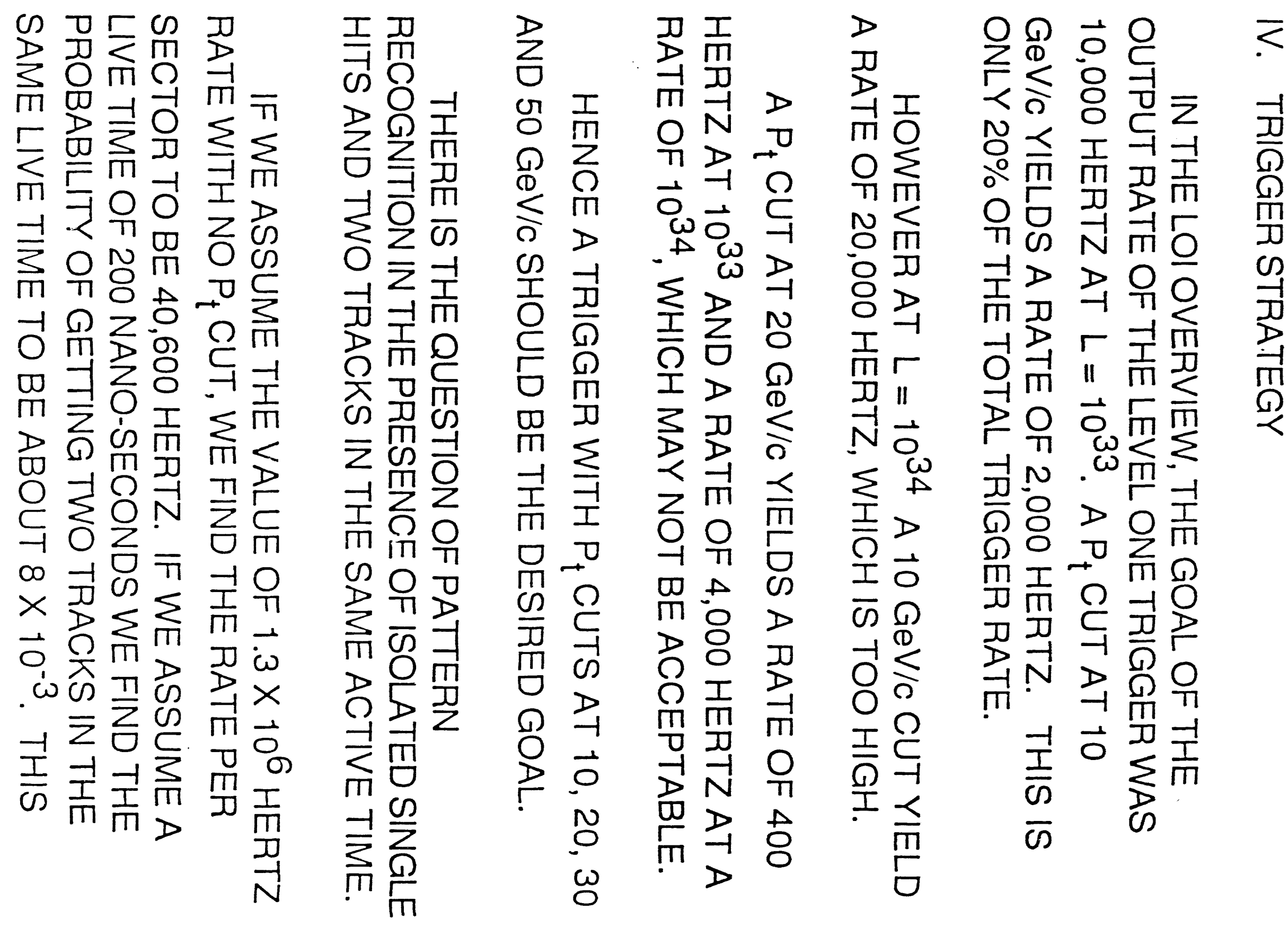




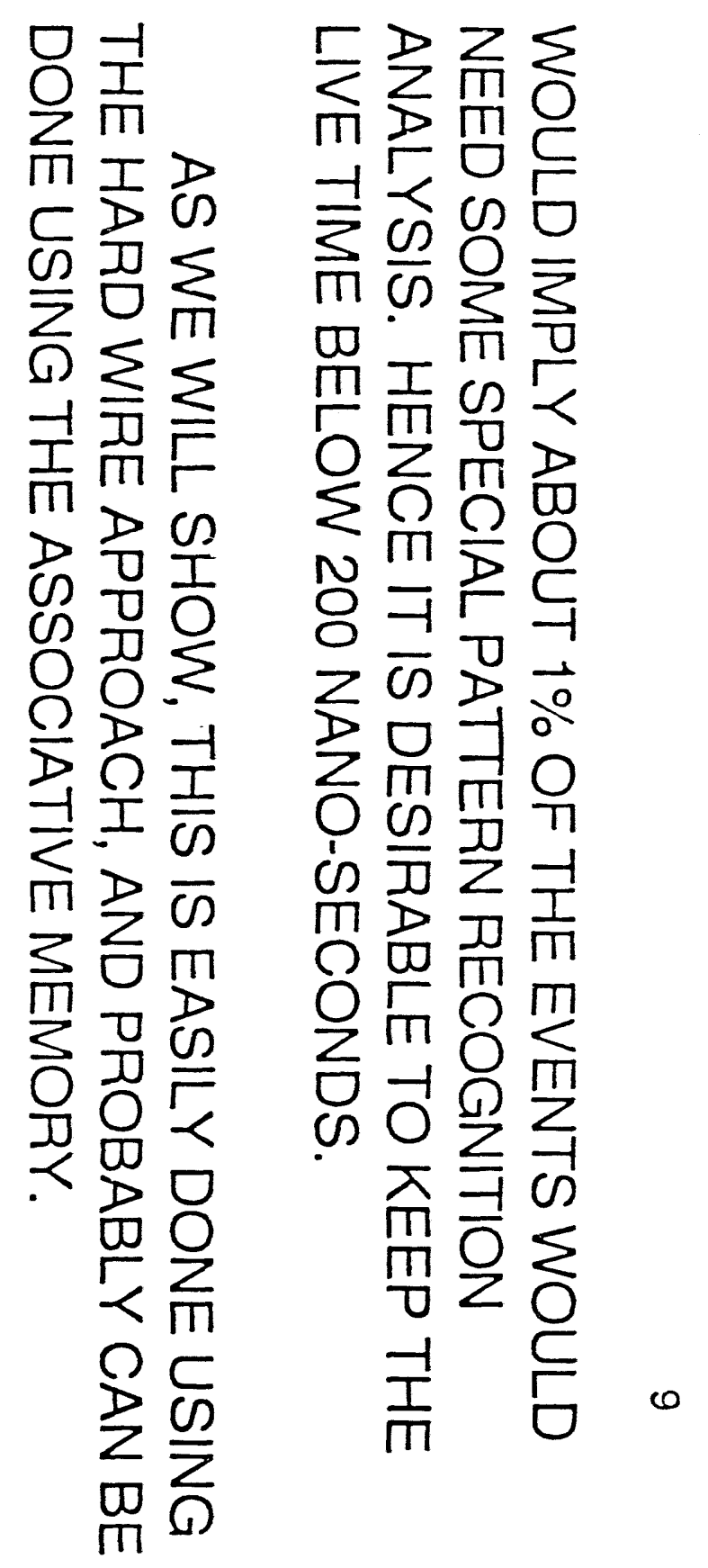




\section{PHESENTATION TO THE GEN WUON THIGGEN SUBGHOUP MHEETING OF $G$ JAINUARY 1992 AT BINL \\ IRWIIN A. PLESS}

BASE LINE GEOMIEIRY
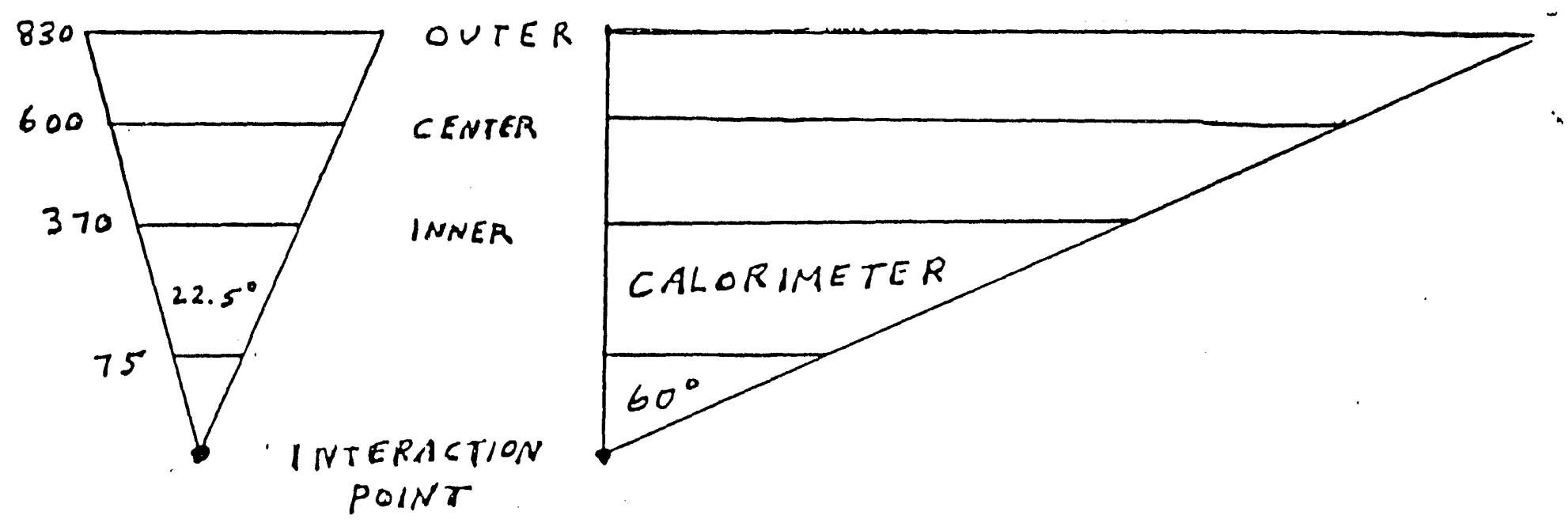

RADIUS LENGIH WIDTH

Outer plane

Center plane

Inner plane

830
600
370

1450

330.2

1048

239.7

664

147.2

CALORIMEIER

Inner radius

Outer radius

Interaction thickness

Total number of radiation lengths

$$
\begin{aligned}
& =75 \\
& =370 \\
& =12 \lambda \\
& =12.56 \times 18.5=396
\end{aligned}
$$

$$
\begin{aligned}
& \theta_{0}=21 / E \sqrt{ } / x_{0}=21 / E \sqrt{ } 396 \\
& \theta_{\text {plane }}^{\text {RivS }}=1 / \sqrt{ } 2 \theta_{0} \\
& \sigma_{\text {plivis }}^{\text {Rlane }}=1 / \sqrt{3} L \theta^{\text {RMS }} \\
& \text { plane }
\end{aligned}
$$




\section{BEND PLANE CALCULAIIONS}

Al! strip widths $=1.3 \mathrm{~cm}$

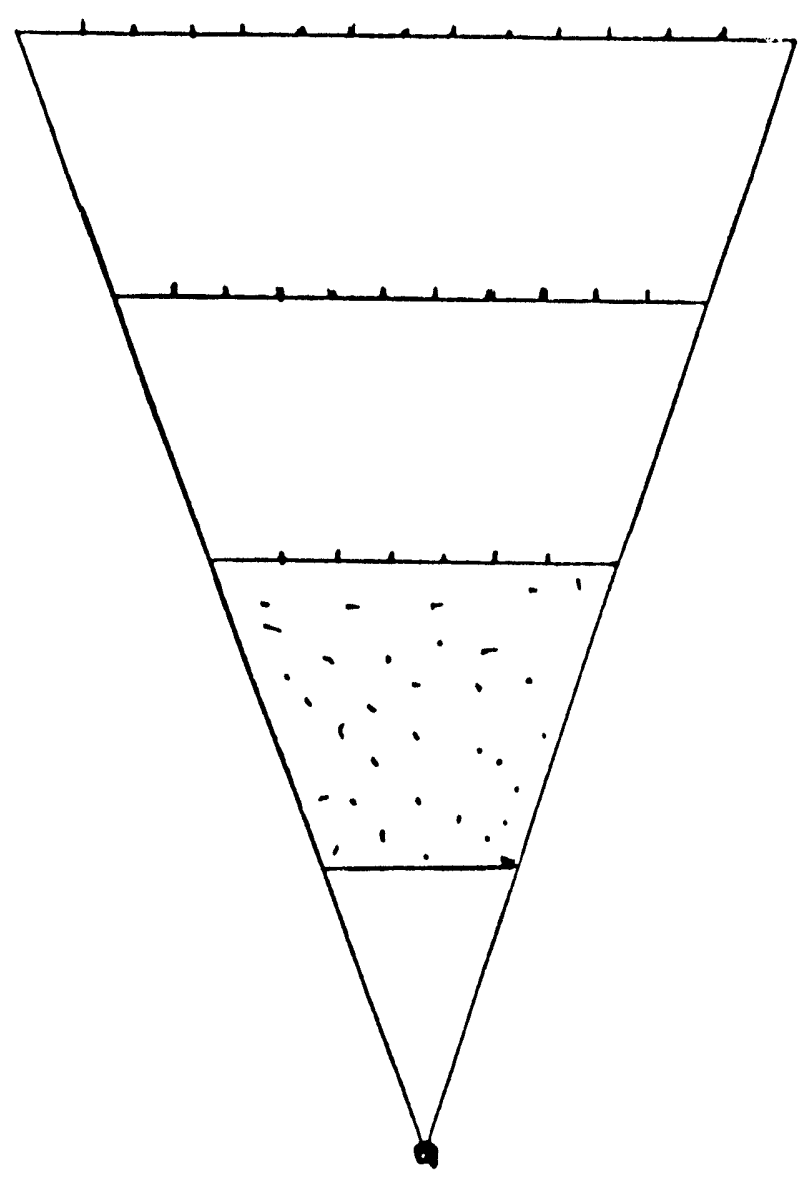

Outside plane Center plane

$=254$ strips

Inner plane

$=185$ strips

$=113$ strips

Total number strips

$=552$ (= number of discriminators) 


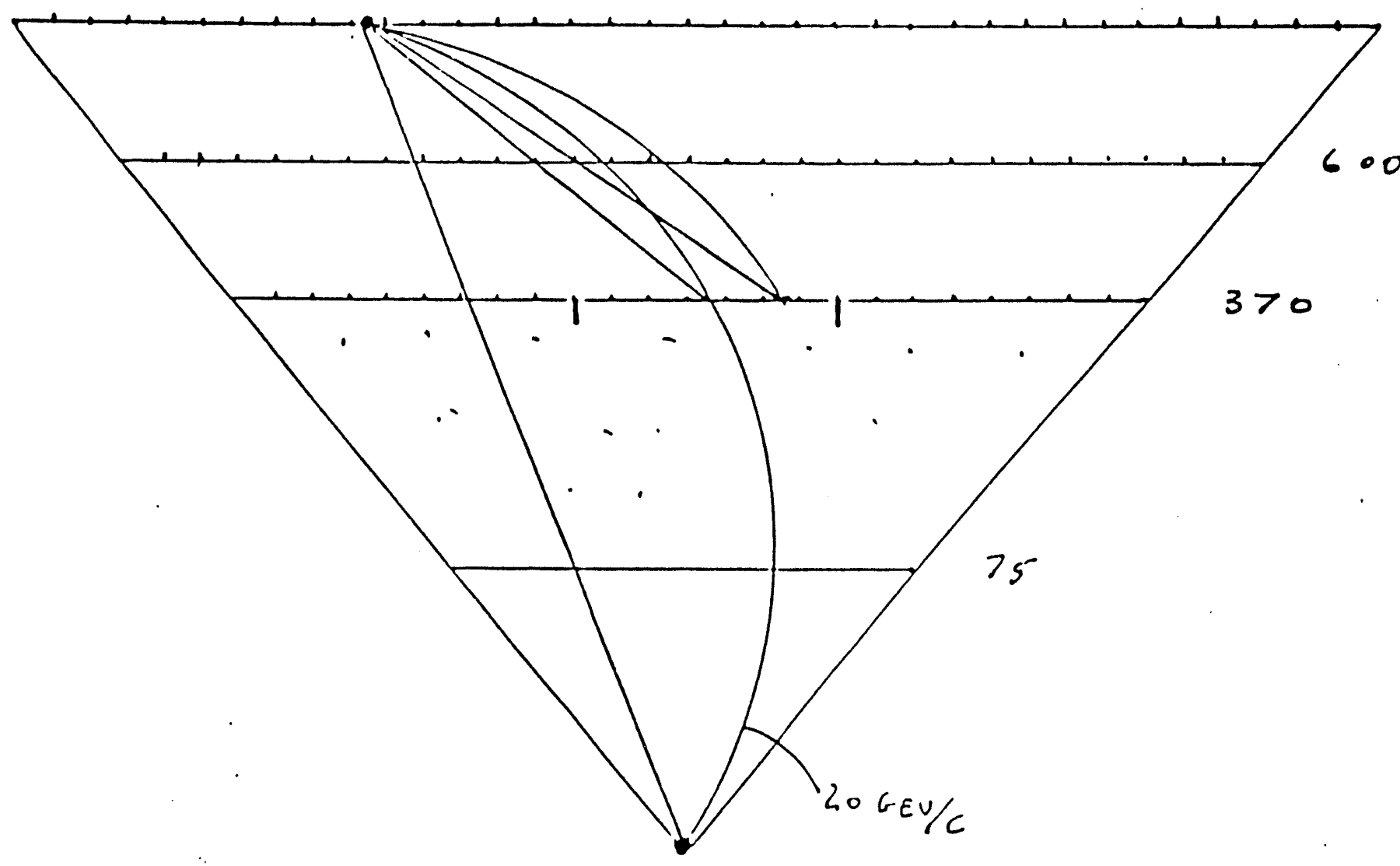

A $20 \mathrm{GeV} / \mathrm{C}$ track has a sigma of 2 strips around the ideal intersection point at $\mathrm{A}=370$ due to multipie scaltering. In order to have a $95 \%$ acceptance we have to be able to handle \pm 4 strips around the ideal (no multiple scaltering) intersection point. 


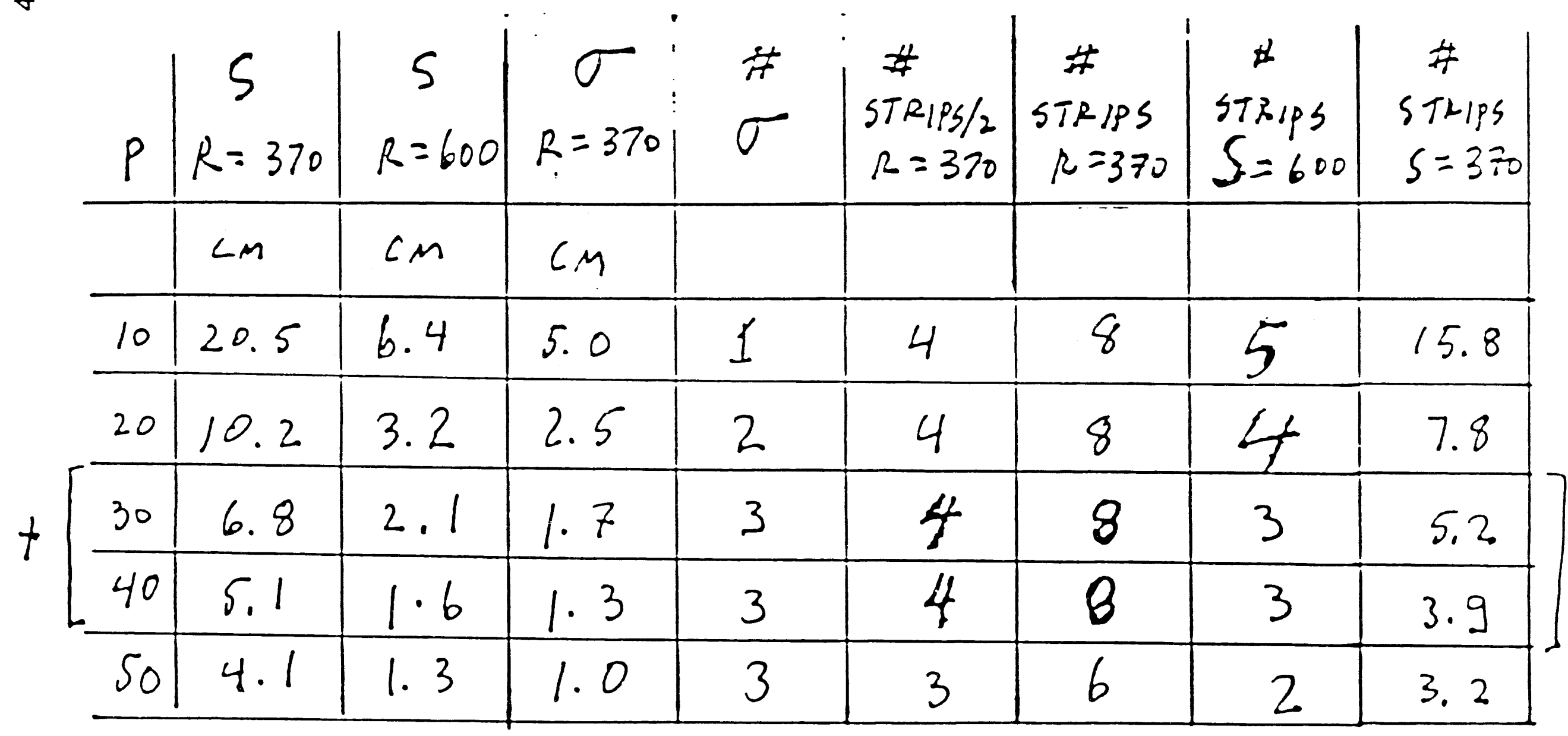

I 30 AND 40 CANNOT BO SEPARATED 
To handle the multiple scattering problem we must have many circuits.

$10 \mathrm{GeV}$ positive charge. For example:

$8 \times 254=2032$

$\begin{array}{ll}10 \mathrm{GeV} & =2032 \\ 20 \mathrm{GeV} & =2032 \\ (30+40) \mathrm{GeV} & =2032 \\ 50 \mathrm{GeV} & =1524\end{array}$

Total number of circuits for positive charge

$=7620$

For both charges $=2 \times 7620$

$=15,240$

The logic gives four momentum trigger ranges.

$>10 \mathrm{GeV} / \mathrm{c}$

$>20 \mathrm{GeV} / \mathrm{c}$

$>30 \mathrm{GeV} / \mathrm{C}$

$>50 \mathrm{GeV} / \mathrm{C}$ 
Circuit "Design" using signelics $100 \mathrm{~K}$ series ECL chip 100101. This is a 5 input and/or chip.

Need a fast infinite fan in circuil (infinity $=2032$ )

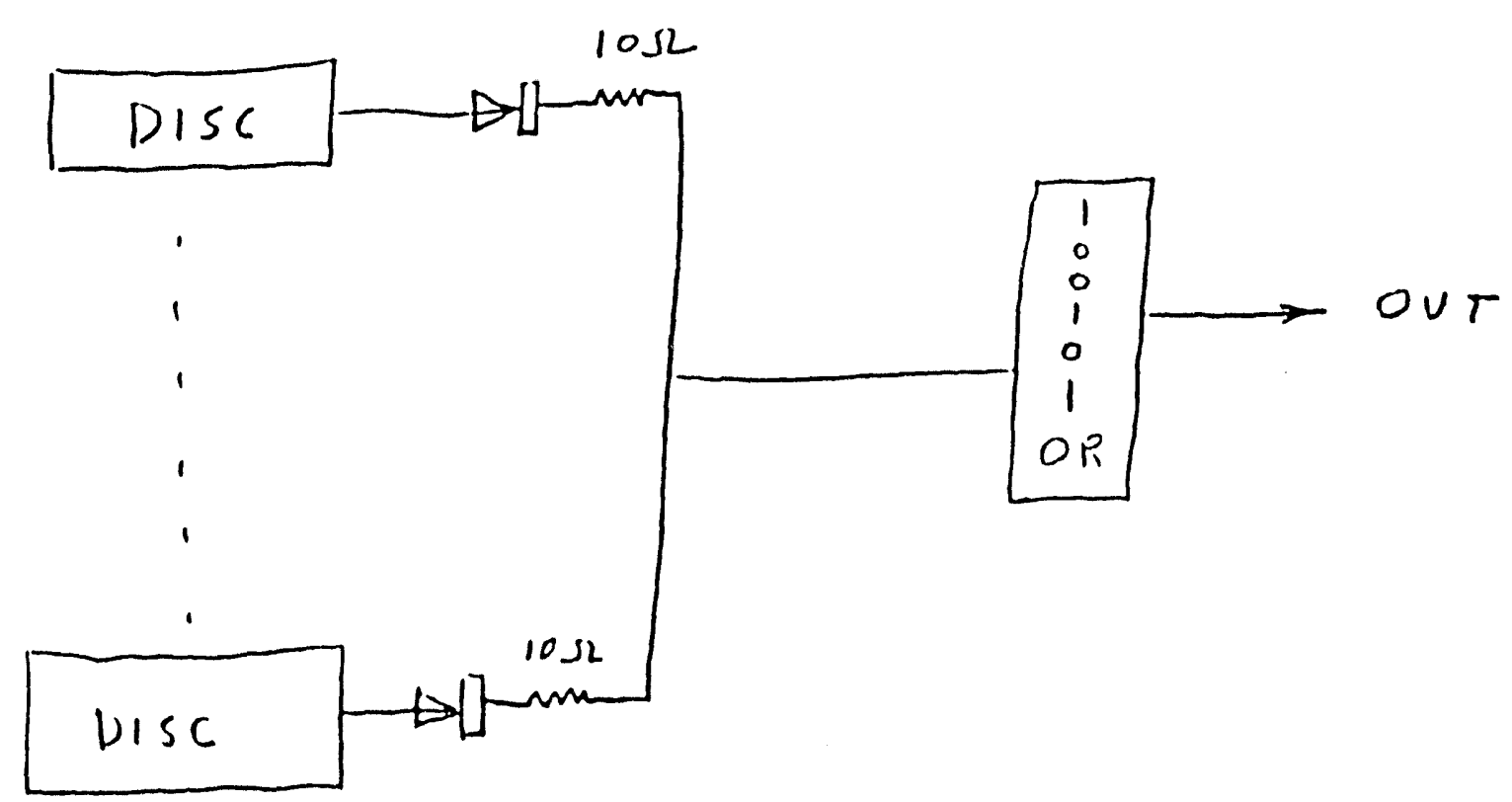

$10 \mathrm{GeV}$ Circuit $=8 \times 2 \times 254=4,064$ circuits $(2,032$ positive; 2,032 negative).

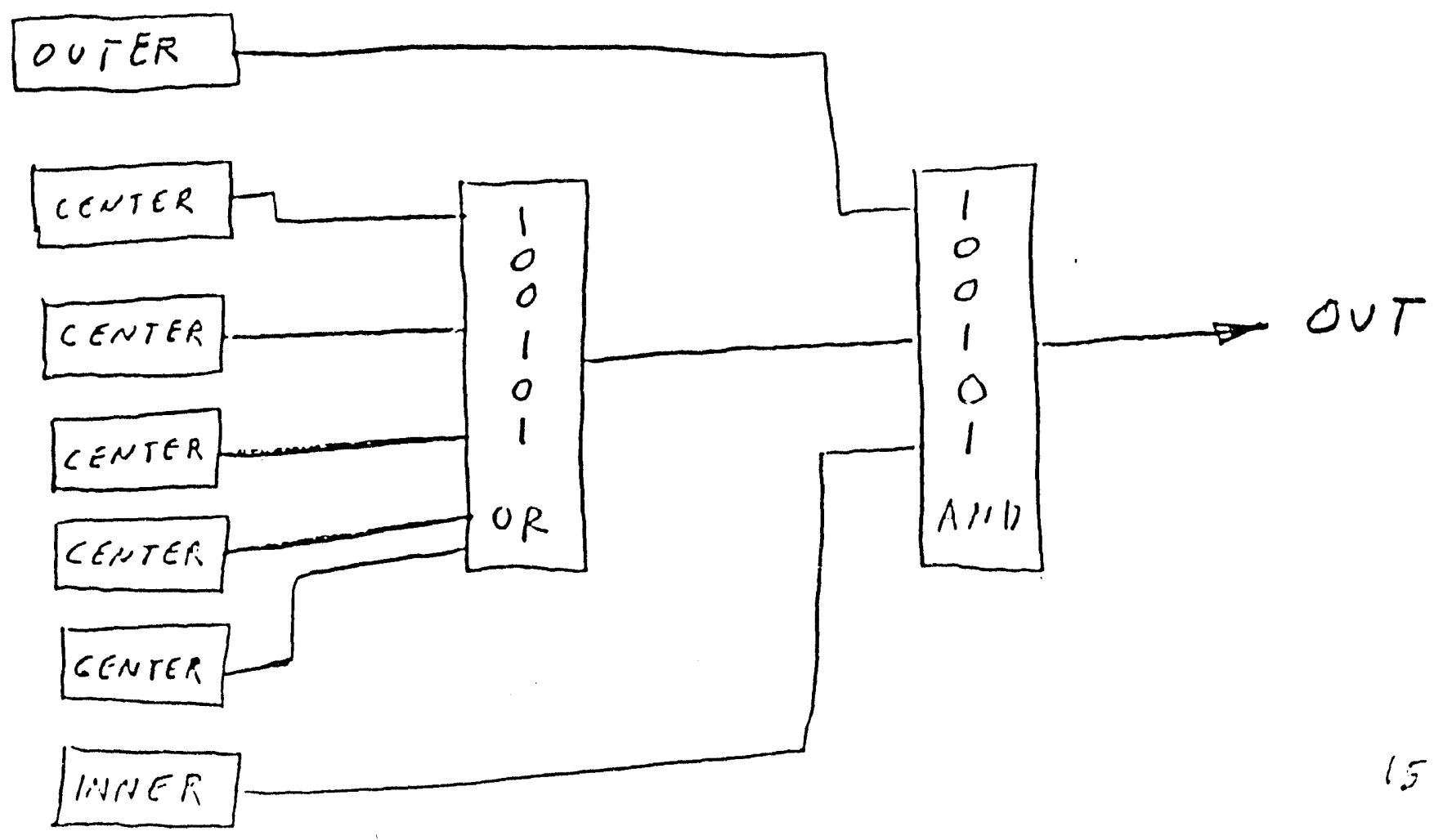


$20 \mathrm{GeV}$ circuit - need 4,064 circuits.

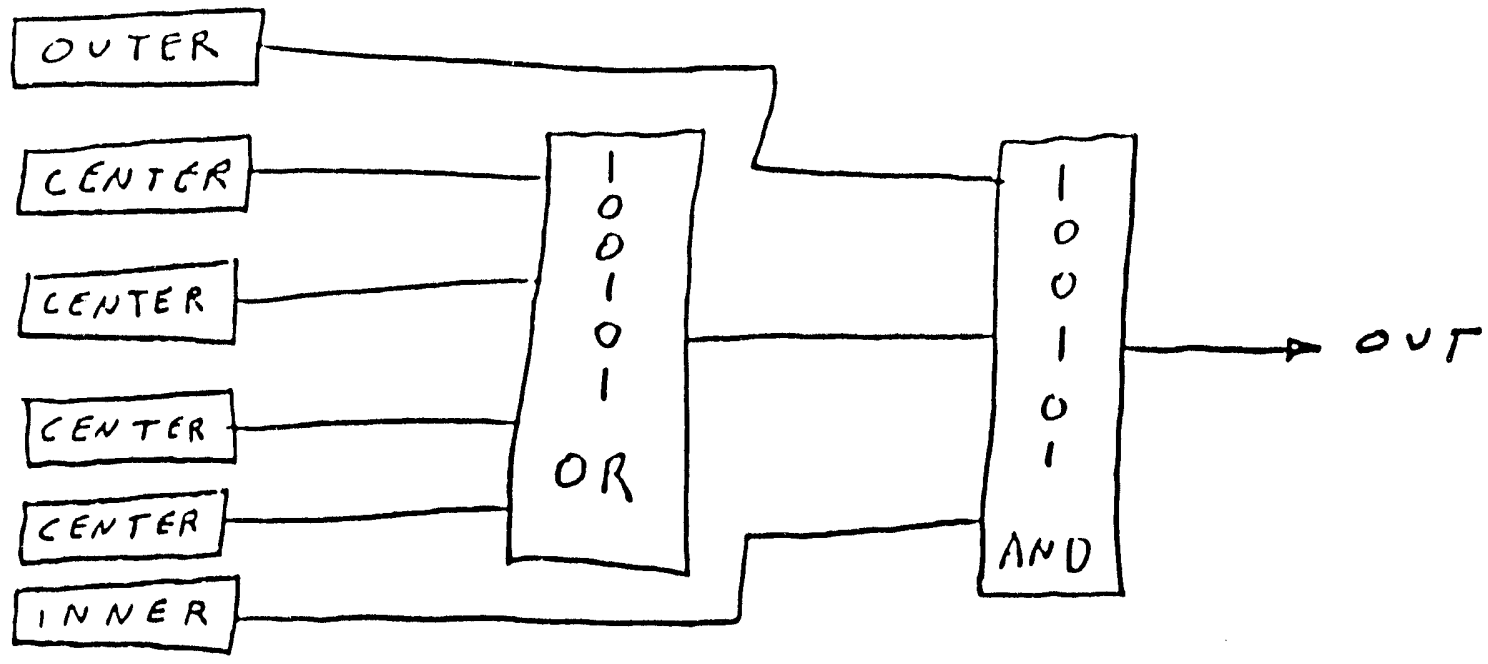

(30-40) GeV circuit - need 4,064 circuits

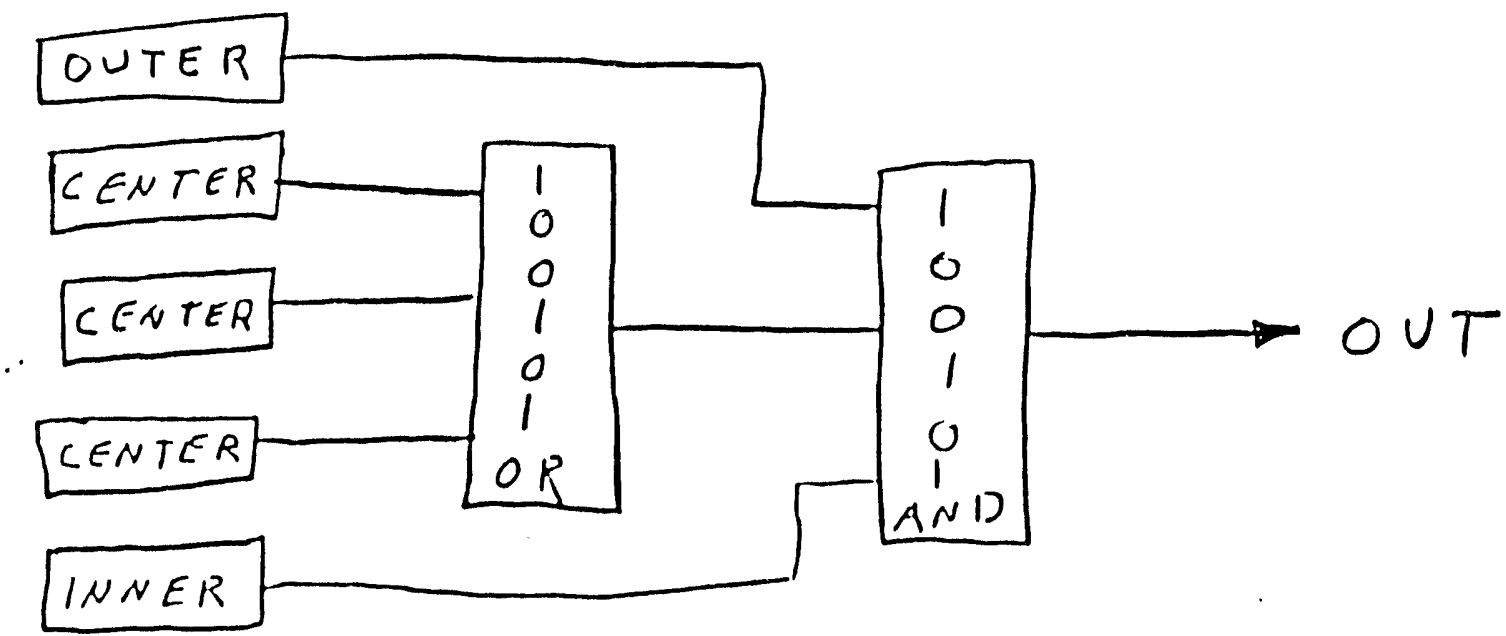

$50 \mathrm{GeV} / \mathrm{c}$ circuit - need $6 \times 2 \times 254=3,048$ circuits (1524 positive; 1524 negative)

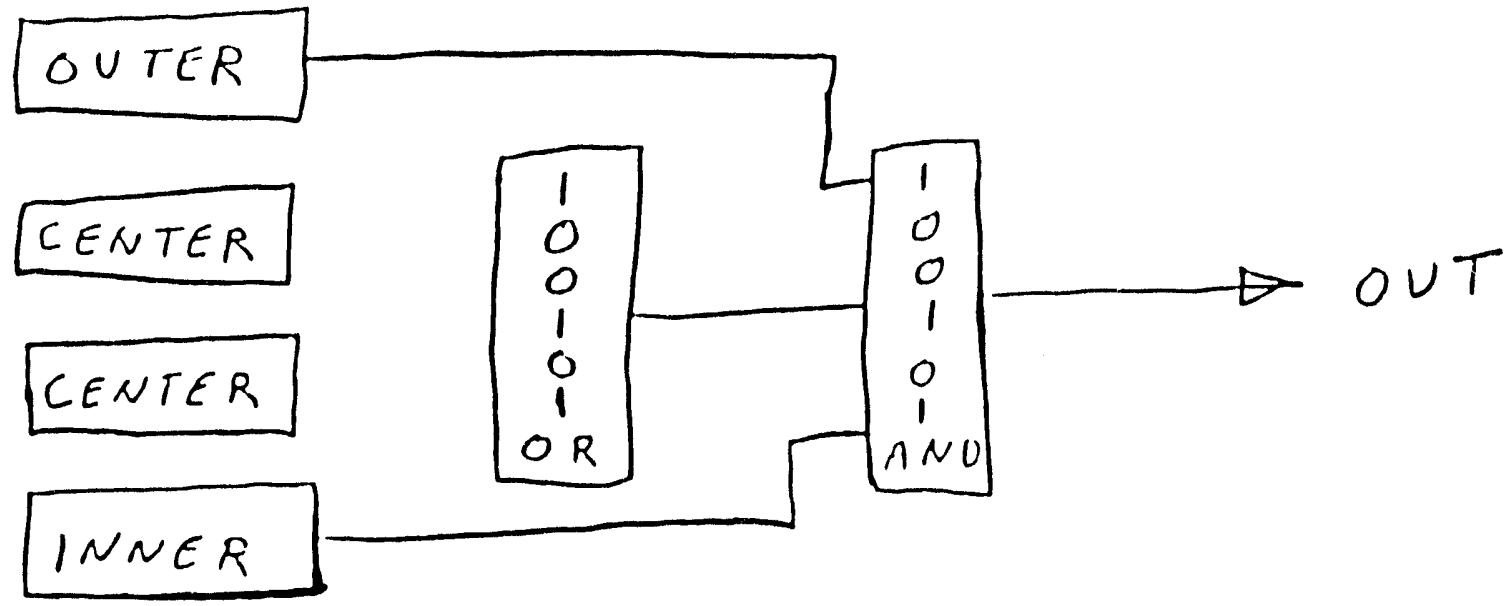


8

POSITIVE MUON

Trigger $>10 \mathrm{GeV}$

NON BEND TRIGGER

DISC
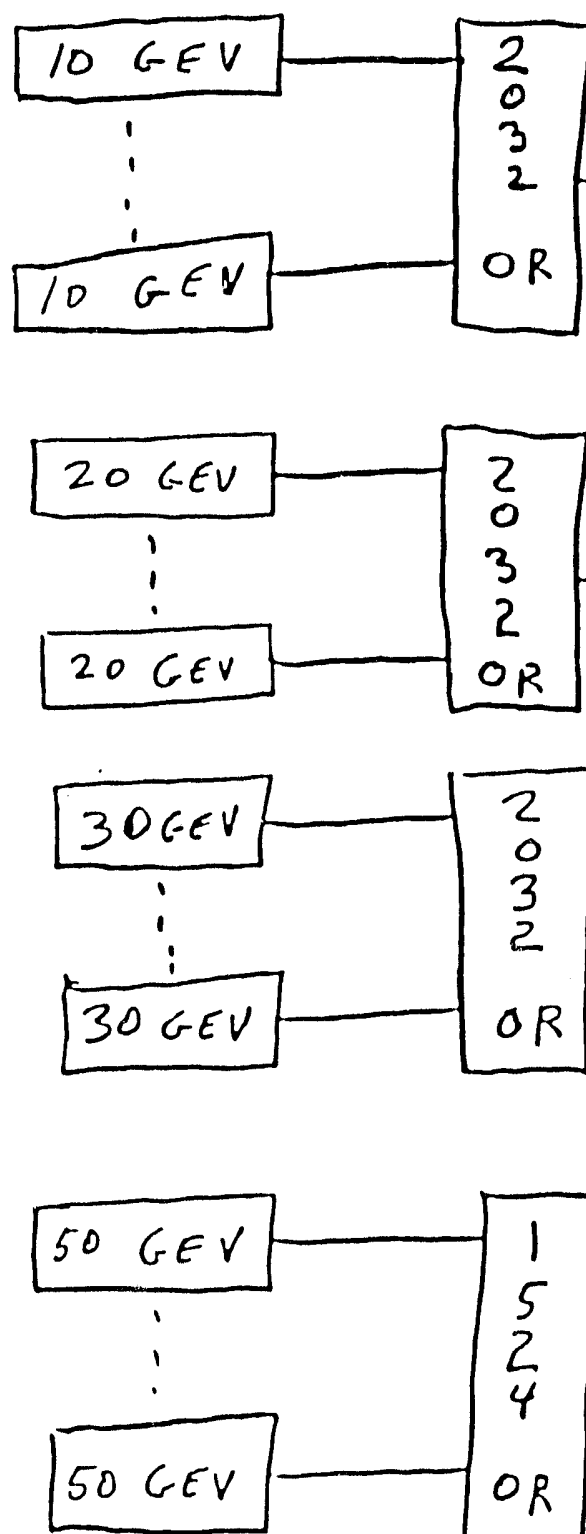

1
0
0
0
1
$O R$

1
0
0
1
0
1

$\wedge N D$

17 
NON BENO IIMGGEH

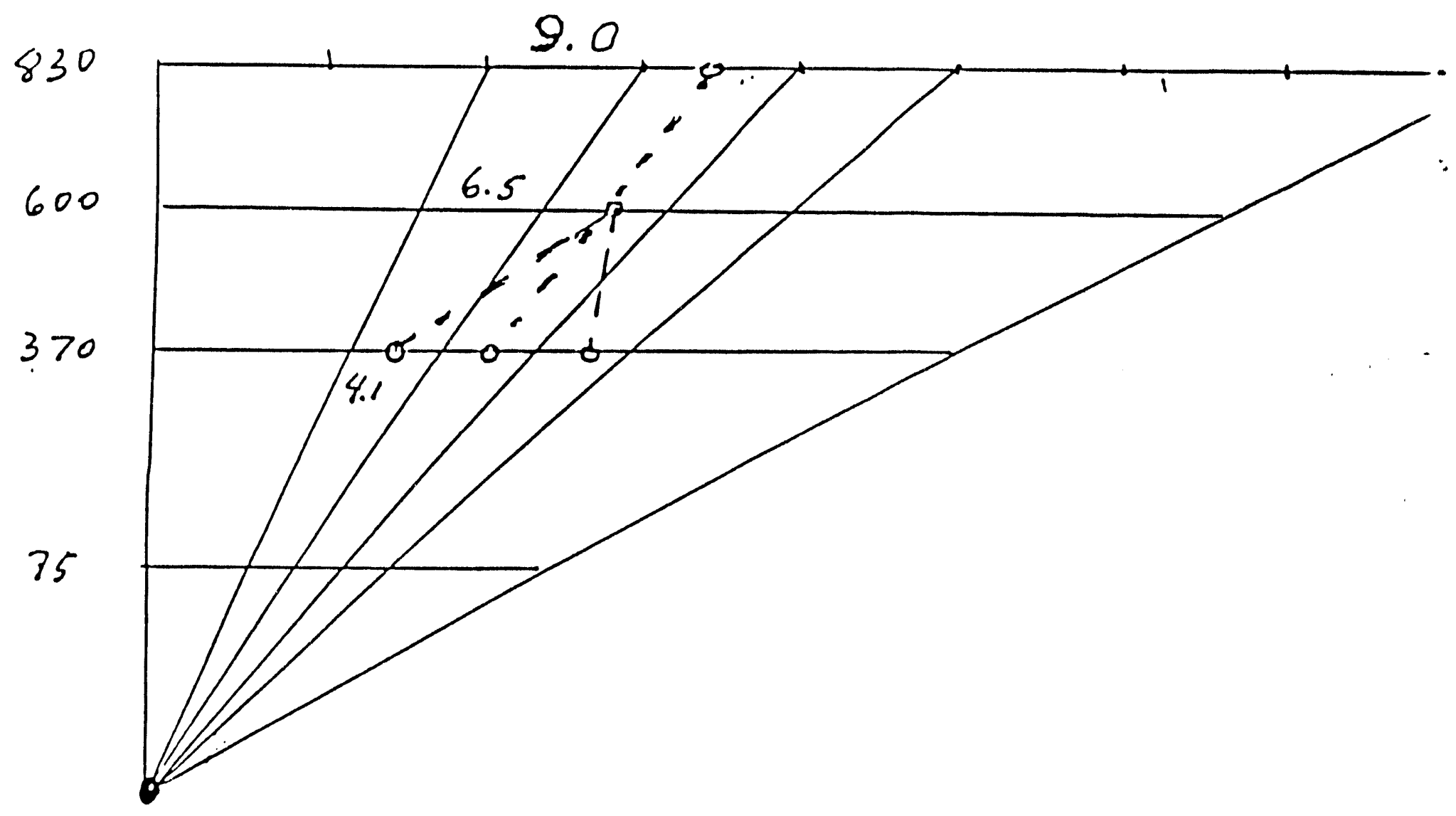

For each outer strip there are three, lliree fold coincidence circuils. Any triple coincidence generales a non-bend trigger. 


\section{NON BEND TRIGGER}

Tower geometry.

Each plane is divided into 161 strips.

$\begin{array}{ll}\text { Outer } & =9.0 \mathrm{~cm} \\ \text { Center } & =46.5 " \mathrm{~cm} \\ \text { Inner } & =4.1 \mathrm{~cm}\end{array}$

Center is really 5 strips OR'd together, each strip is $1.3 \mathrm{~cm}$ wide to furnish the $z$ coordinate of the track.

For each outer strip there are three coincidence circuits to account for multiple scallering.

This implies there are $3 \times 161=483$ circuits per sector. Each circuit is as follows:

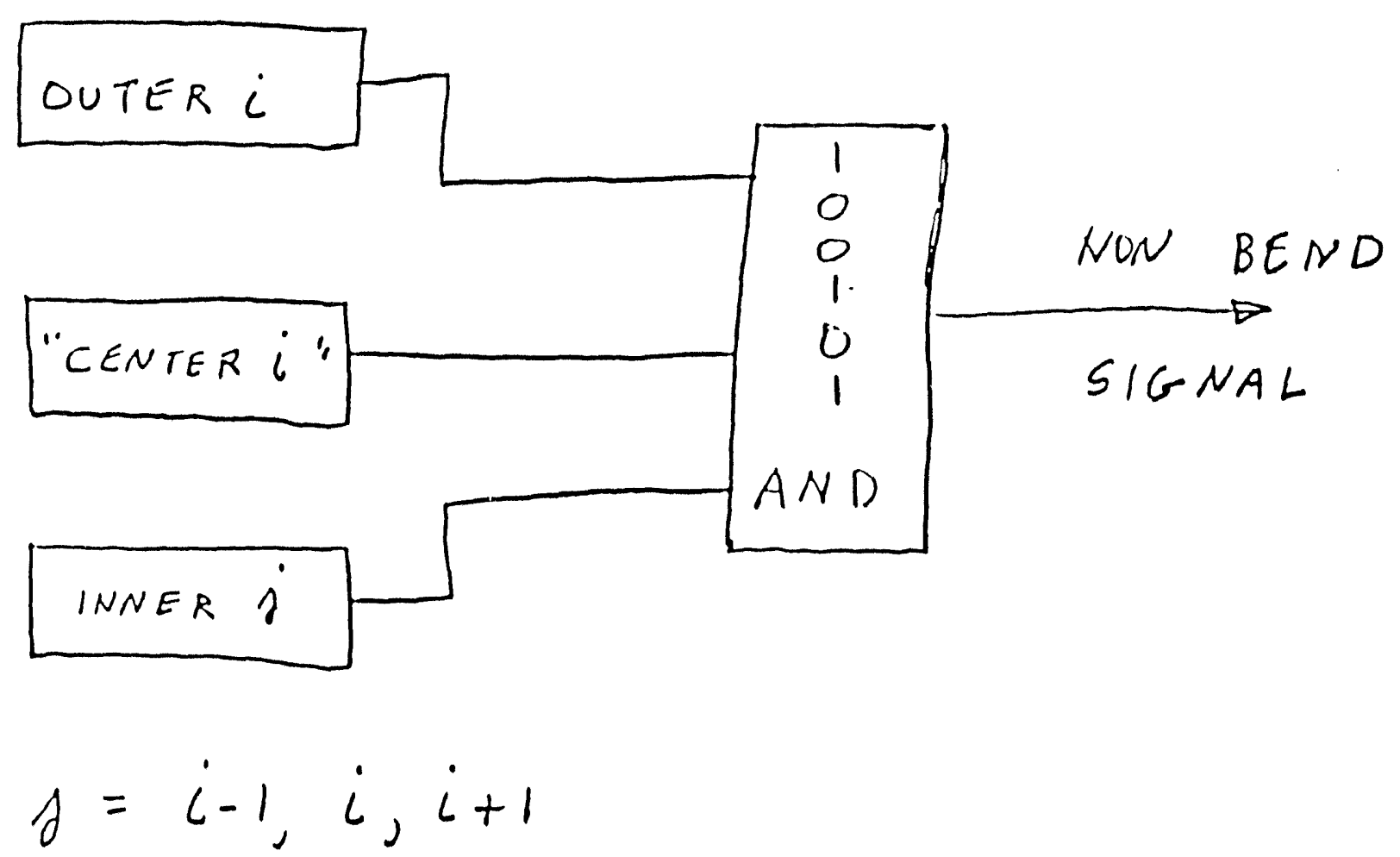


NON BEND CIRCUIT

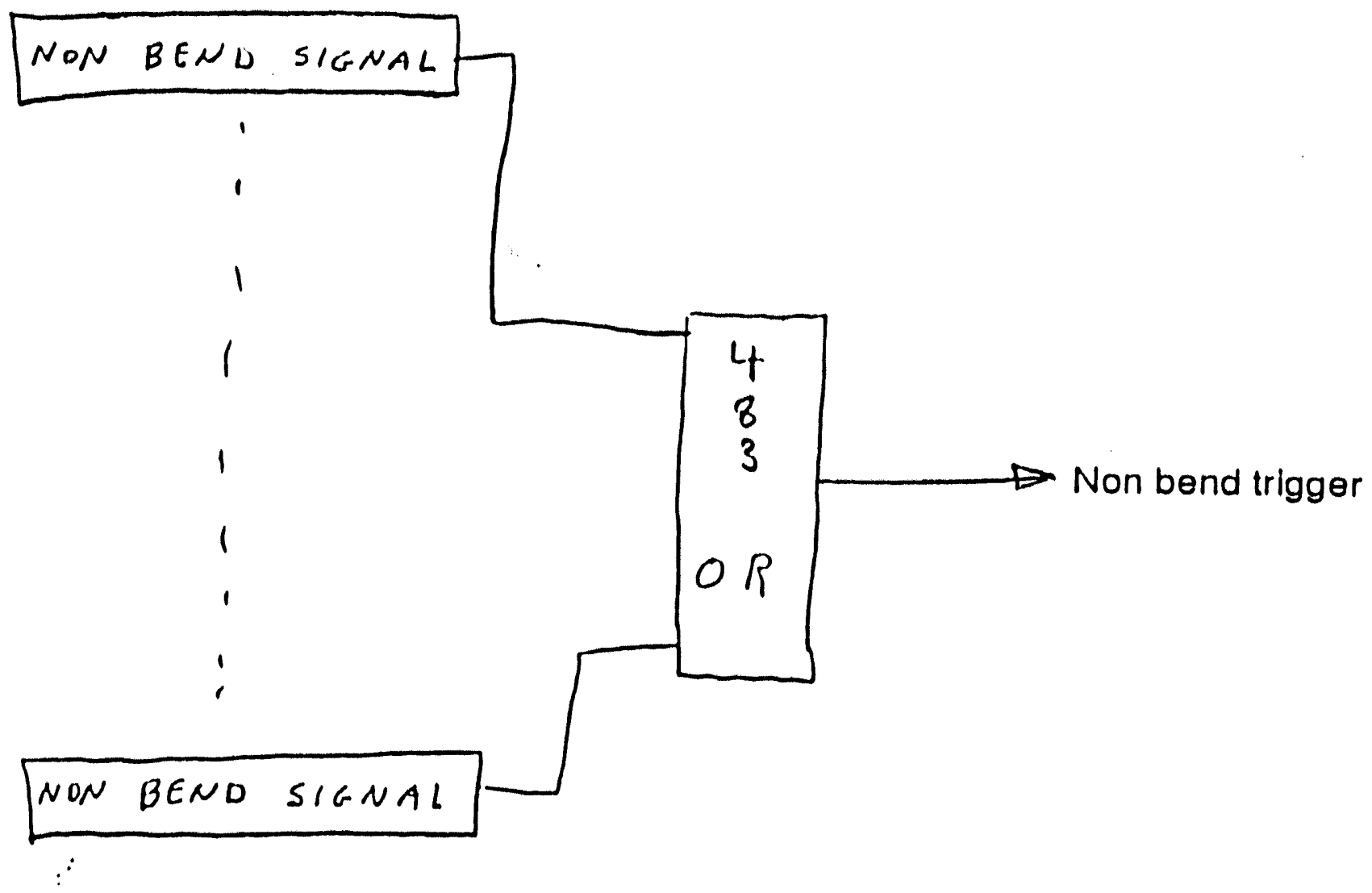

This has $100 \%$ acceplance for $P_{t}>30 \mathrm{GeV} / \mathrm{c}$.

This has about $70 \%$ acceplance for $P_{t}=10 \mathrm{GeV} / \mathrm{c}$

Acceplance falls rapidly for $P_{t}<10 \mathrm{GeV} / \mathrm{C}$

We have

$$
\begin{array}{ll}
161 \text { strips } & 4.1 \mathrm{~cm} \text { (inner) } \\
161 \text { sirips } & 9.0 \mathrm{~cm} \text { (outer) } \\
805 \text { strips } & 1.2 \mathrm{~cm} \text { (center) }
\end{array}
$$

Hence a total of 1127 strips $\Rightarrow 1127$ discriminators per seclor. Trigger box must accommodate 1127 connectors or about $2254 \mathrm{~cm}$ linear length. 
SUIVIIIARY

Strip count

$\begin{array}{rrr}\text { Non Bend } & 1,127 \\ & \text { Bend } & 552\end{array}$

Circuits

Non Bend

483

Berid

15,240

Chips

Non Bend 644

Bend $\quad 45,720$

Each discriminator must connect to a maximum of 70 circuits. This puts either a constraint on the output impedance of the discriminator or a series input resistor to the $100101 \mathrm{ECL}$ chip. As an alternative, to save power and money, all this can be redone in C-MOS with large scale integration techniques.

Question:

How do we write the simulation program? 


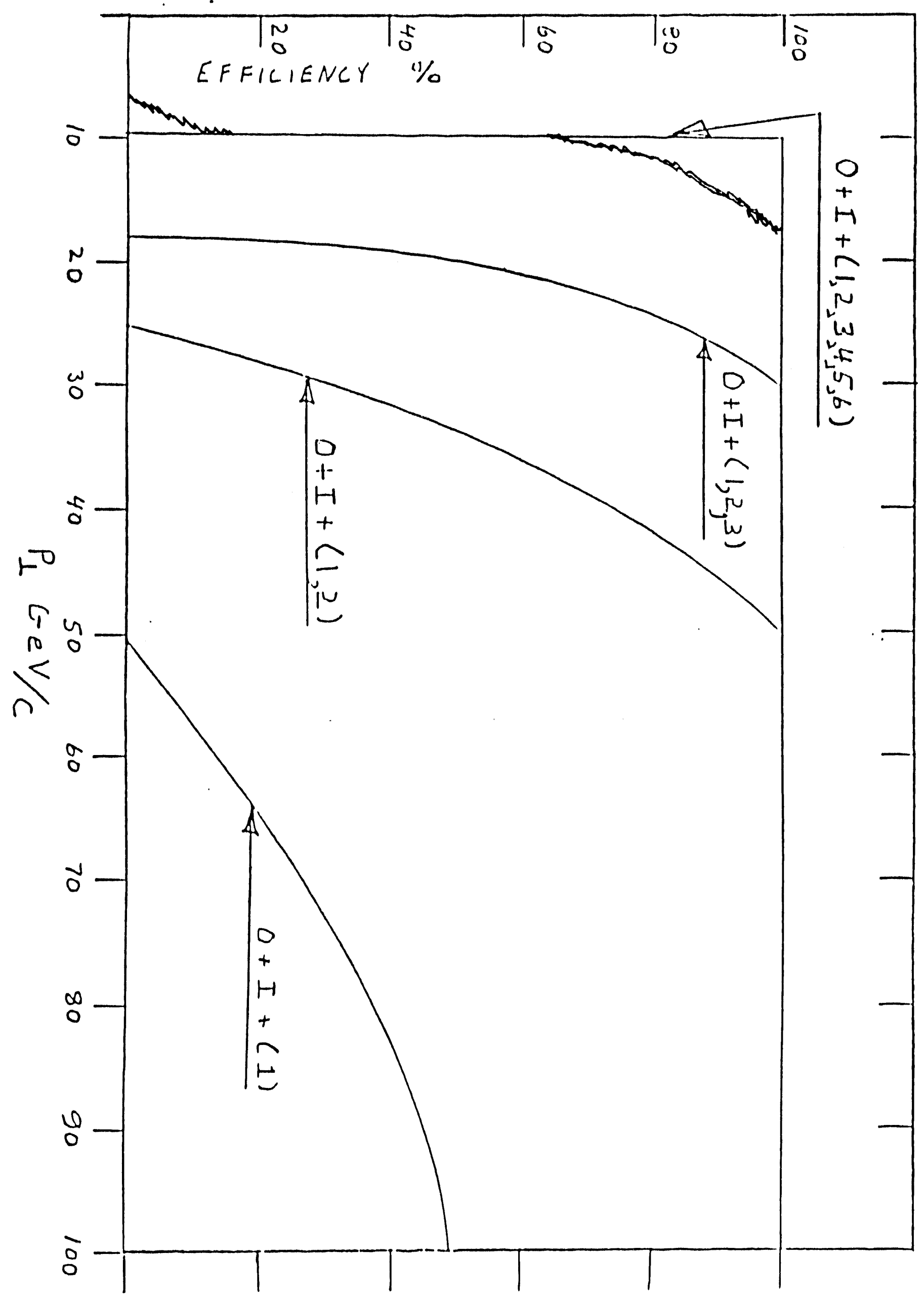


V. HARDWARE IMPLEMENTATION

THE FIRST QUESTION WITH RESPECT TO HARDWARE, IS HOW DO YOU GET THE SIGNALS OUT TO THE COMMON ELECTRONICS POINT. IN OUR CASE WE PROPOSE TO BRING THE SIGNALS OUT ALONG THE EDGE OF THE RPC LAYERS AT THE LAYER LEVEL AND ALONG THE 60 LINE TO THE OUTER LAYER FOR THE INTER LAYER CONNECTIONS.

FOR EACH LAYER WE HAVE 161 CABLES RUNNING ALONG THE EDGE OF THE LAYER, USING RG 58 CABLE. THIS IS A CABLE BUNDLE 5.8 INCHES ON A SIDE.

FOR THE RUN FROM THE INNER LAYER TO THE CENTRAL LAYER WE USE 274 CABLES. THIS IS A CABLE BUNDLE OF 7.5 INCHES ON A SIDE.

FOR THE RUN FROM THE CENTER LAYER TO THE OUTER LAYER WE HAVE 1,264 CABLES. THIS IS A CABLE BUNDLE OF 16 INCHES ON A SIDE.

ALTERNATELY, WE CAN RUN ALL CABLES IN A FLAT PACKAGE TWO INCHES WIDE. THAT WOULD GIVE WIDTHS OF 17, 28.2, 128 INCHES WIDE, FOR ALONG THE LAYER, FROM THE INNER LAYER TO THE CENTER LAYER AND FROM THE CENTER LAYER TO THE OUTER LAYER RESPECTIVELY. 
VI. ASSOCIATIVE MEMORY IMPLEMENTATION

AN ASSOCIATIVE MEMORY OPERATES BY HAVING STORED HIT PATTERNS AND THEN FOR EACH INPUT PATTERN, COMPARING EACH OF THE STORED PATTERNS WITH THE HIT PATTERN

THE NUMBER OF PATTERNS WE HAVE IS:

$\begin{array}{lr}10 \mathrm{GeV} & 12,880 \\ 20 \mathrm{GeV} & 10,304 \\ 30 \mathrm{GeV} & 7,728 \\ 50 \mathrm{geV} & 3,864 \\ & \\ \text { TOTAL } & 34,776\end{array}$

SINCE EACH ASSOCIATIVE MEMORY CAN HANDLE 128 PATTERNS, WE NEED ONLY 272 SUCH MEMORIES. HOWEVER EACH MEMORY CONTAINS OVER 120,000 CIRCUITS. HENCE WE ARE TALKING ABOUT $9 \times 10^{6}$ CIRCUITS PER SECTOR.

HOWEVER, THESE SEEMS TO BE A FAIR AMOUNT OF EXTERNAL LOGIC THAT REQUIRES ADDITIONAL CIRCUITS, AND TIME, IN ORDER TO UTILIZE THIS APPROACH. IN PRINCIPLE, HOWEVER, IT SEEMS THAT ONE CAN USE THE ASSOCIATIVE MEMORY SCHEME FOR THE RPC TRIGGER. 


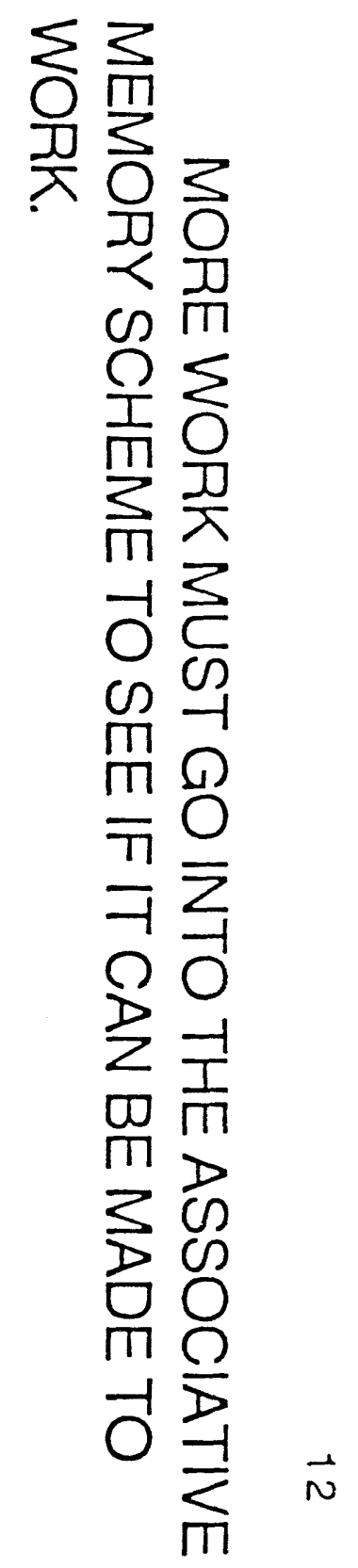




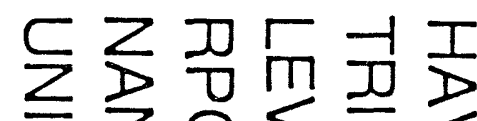

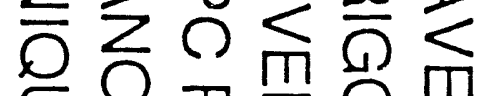

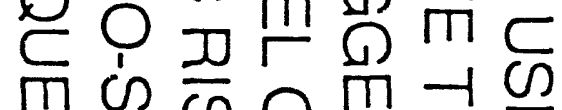

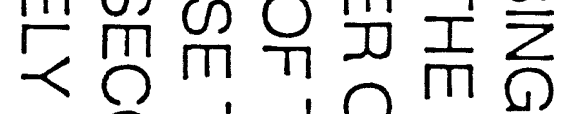

으ㄹㅡㅓㅓㄴㄲㄲㄲ응

男方而卫

$\exists \Theta \subseteq D \unrhd \cong$

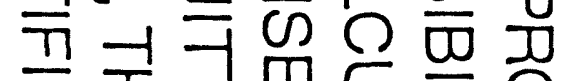

而开网

而而与可

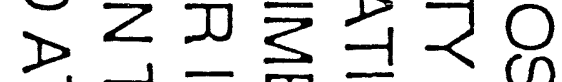

本我而文吕

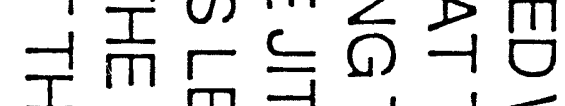

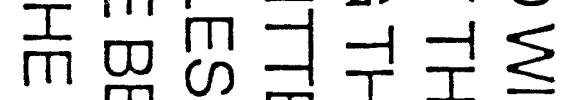

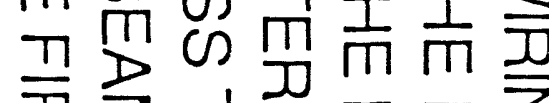

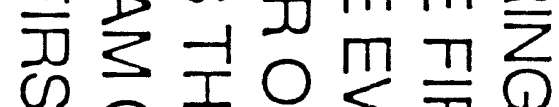

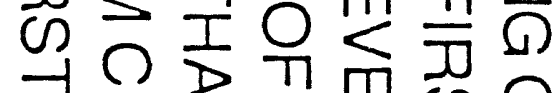

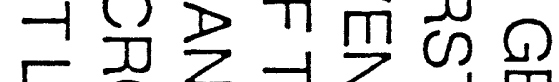

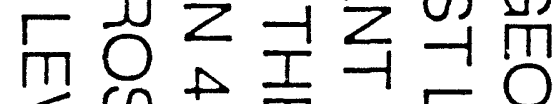

$<\omega^{\circ}$ 而

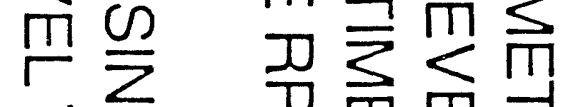

式迳
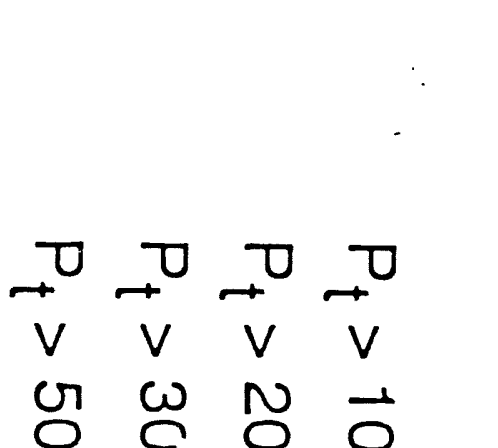

(]) $\Omega \Omega$ $\stackrel{\infty}{<} \stackrel{\infty}{<} \stackrel{\infty}{<}$

$\leqslant \leqslant \leqslant \leqslant$

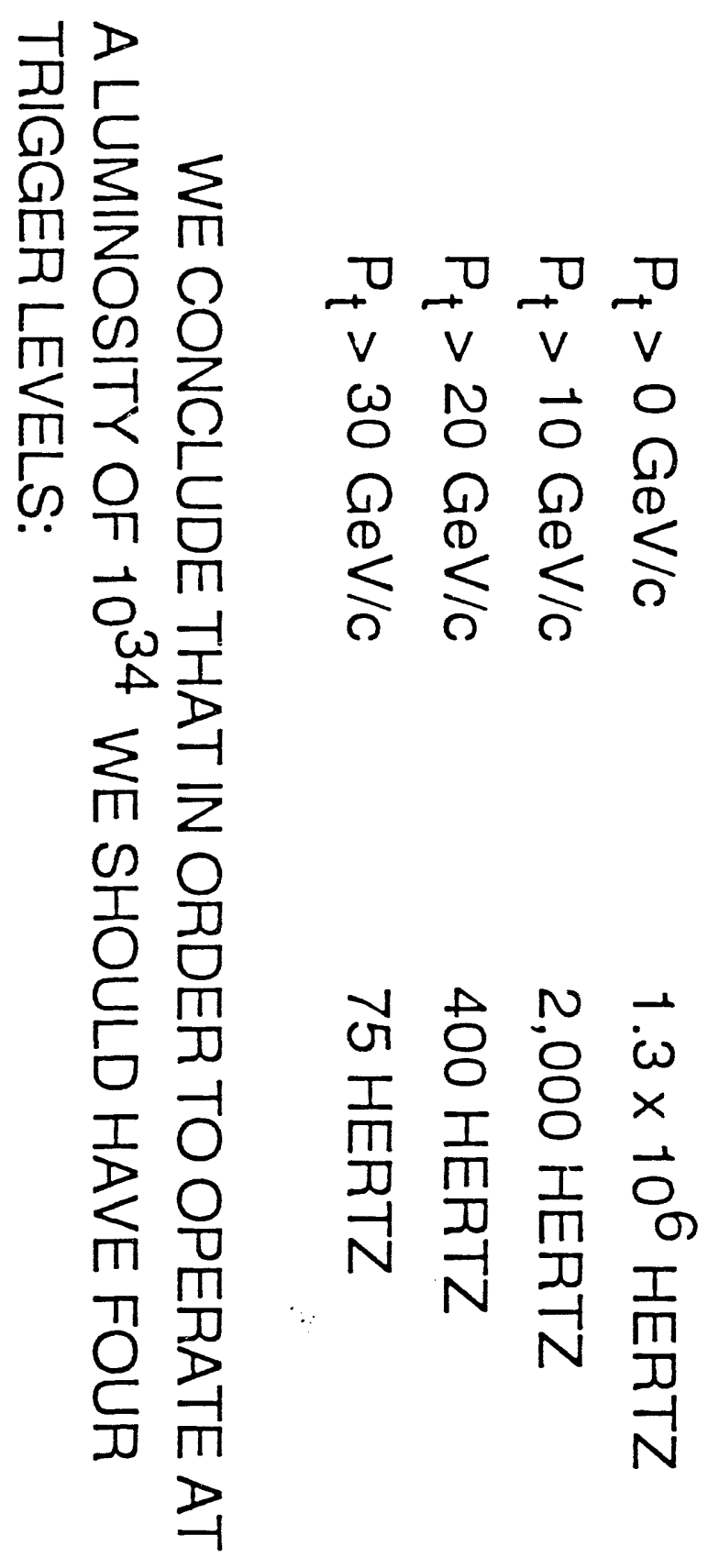

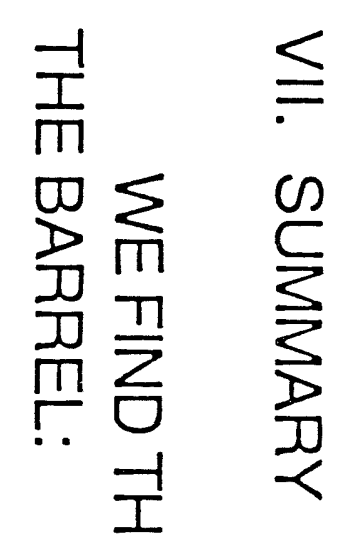

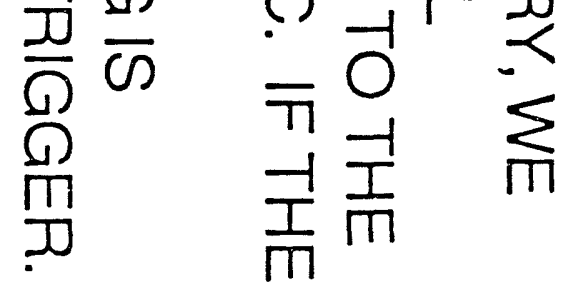




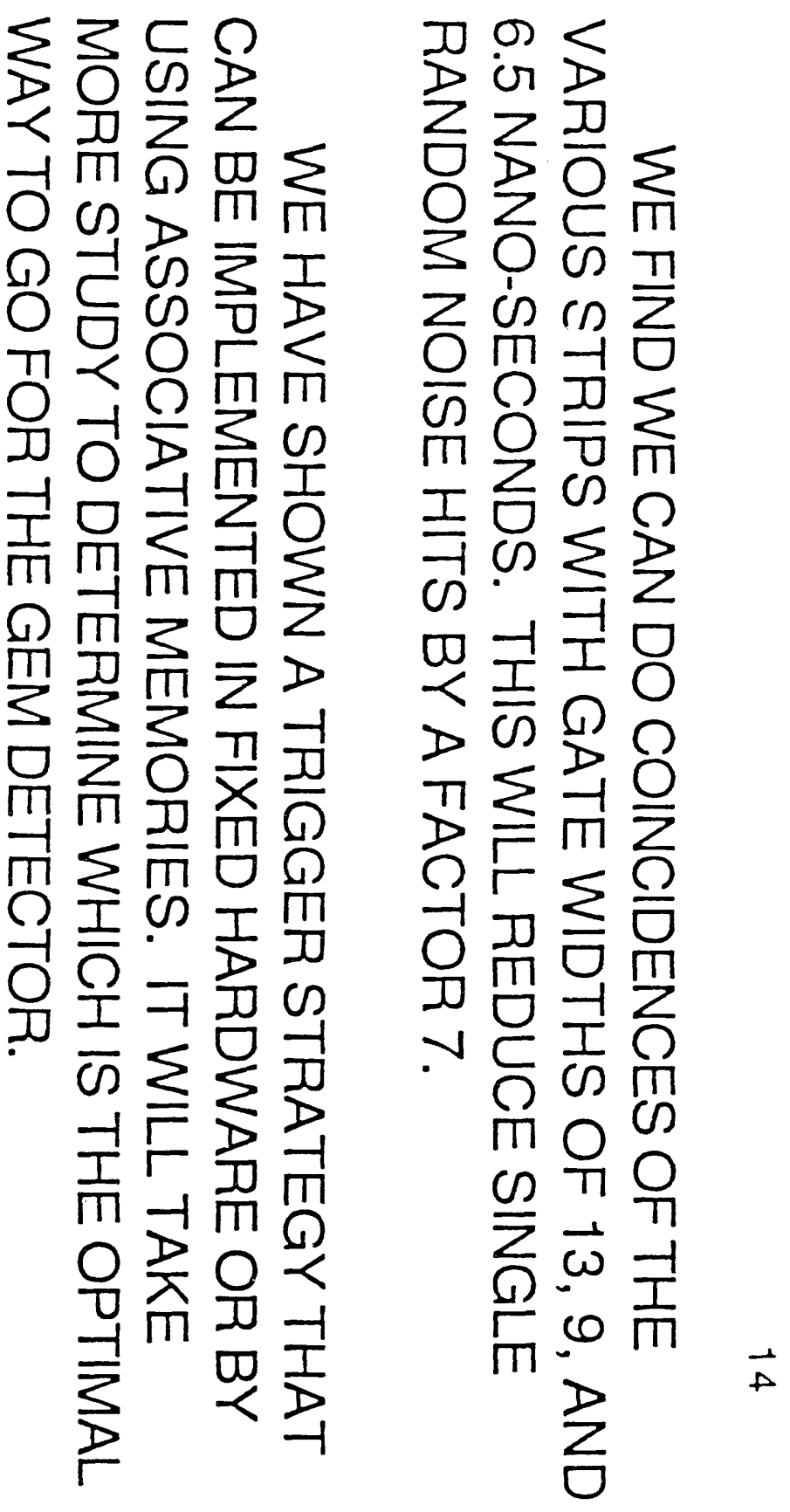




\section{APPENDIX 2 \\ Meantimer (chronotrons) technique for use with Resistive Plate Counters \\ D. Marlow}

June 10, 1992 
It\# SอยโรL

โ2:9108/60

High Energy Physics Group Princeton University Jadwin Hall

Post Office Box 708

Princeton, New Jersey 08544

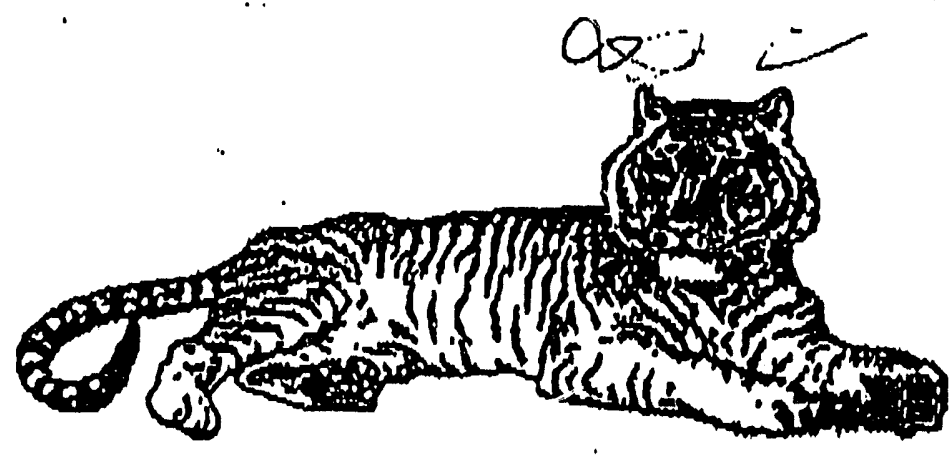
U.S.A.

Telefax: 609-258-6360

Telex: 499.3512

To: Prot. Irwin Pleas

From: Dan Harlow

Date: 6/10/92 Time: $16^{38}$ EDT

Nurnber of pages: 5 plus cover

Number called: $\quad 617 \quad 565 \quad 8316$ 


\section{こャ\#

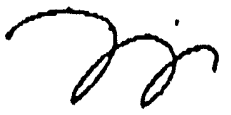

โ2:9โ $0 \varepsilon / 60$

Duniel Marlow

Juse 9, 1992

\section{A Simple Circuit for Time-Deskewing in the GEM RPC's}

\section{Introduction}

The baseline option for the barrel muon trigger system in GEM is the Resistive Plate Chamber (RPC). The good timing resolution $\left(\sigma_{t} \simeq 1-2\right.$ na) of these devices makes realtime bunch-cross tagging possible. This feature helps to simplify many other aspects of the trigger and DAQ system design. Practical complications arise, however, becaure of the large size of the GEM muon aystem, which produces considerable variations in arrival times and pulse trassit times along the readout strips.

As an example, consider the simple sketch of Figure 1, which approximately corre. aponds to an outer-layer RPC in the GEM barrel. Muons leaving the vertex at right angles to the beam (trajectory A) travel about nine meters and reach the RPC after roughly 27 ns (to keep the numbers simple, we assume muon flight times $3 \mathrm{ng} / \mathrm{m}$ ). Although the RPC avalanche develops quickly, the resulting current puloe must propagate to the readout electronics located at the end of the strip, as shown. The total delay is then $27+75=102 \mathrm{~ns}$. In constrast, muons leaving the vertex at the most forward angles (trajectory D) must travel $\sqrt{9^{2}+15^{2}}=17.5$ meters, resulting in a pulse-arrival time of $53 \mathrm{nB}$. The $50 \mathrm{~ns}$ difference in arrival times corresponds to three bunch crosoing intervals, which is unacceptable.

\section{Possible Solutions}

Perhaps the most straightforward way to address this problem would be to segment each strip in $z$ into lengths short enough to reduce the time variations to less than $16 \mathrm{~ns}$. In practice thia would require segmenting the 15-meter-long strip shown into four pieces. Unfortunately, this simple expedient raibes the channel count and the attendant electronics costs by a corresponding factor. Other schemes, where the z strips are used to dynamically generate time corrections, have also been proposed, but these are complex and involve 250 $\mathrm{MHz}$ logic.

\section{Meantimers for Long Scintillators}

A similar problem arises in the readout of long scintillators, where transit time varistions produce significant timing jitter if only one PMT is used. However, the average time of arrival of pulses from a pair of phototubes arranged so to view each end of a long scintillator (see top part of figure 2 ) is independent of the particle's impact position. In practice, the average arrival time can be generated in real time using either analog or digital circuits, called "meantimers" or "chronotrons". A classic digital approach employing tapped delay lines is shown in the lower portlon of figure 2. If the total length of cach delay line is chosen to be the same as the time required for light to propagate the length of the counter $(\Delta T)$, the output marked "MT" will fire after a fixed delay time $\Delta T$, independent of the position of incidence. The spacing between taps ja constant and the number of taps (and the number of AND gates) can be adjusted to give the desired resolution. $A$ tap opacing of $\delta t$ limits the single-messurenent error to $\pm \delta t / 2$. 


\section{Application of Meantimers to RPC's}

A variant of the chronotron technique can be applied to the RPC problem. The spacing between taps may be adjusted so as to simultaneously correct for the difference in muon flight times. This is illustrated in figure 3 and the accompanying table. From the table it is evident that latest arrival time occurs in Case $D$, where the arrival time at the left (L) end is $128 \mathrm{~ns}$. The spacing of the taps for any other case is then determined so all other pulses see that same total delay-i,e.

$$
\text { muon flight time + RPC propagation time + delay - line time }=128 \mathrm{~ns}
$$

The choice of four coincidence taps is made for purposes of illustration only. In practice, finer tap spacings would be employed so as to minimize this source of jitter. Even with the relatively coarse tap spacing shown the spread in leading-edge times at the chronotron output is less the $16 \mathrm{~ns}$ spacing between bunches.

\section{Implementation}

The large number ( $33 K$ pairs) of channels contemplated for the RPC system, renders cable delays, or even lumped delay elements, impractical. However, techniques employed in the design of FLASH-TDC's, where CMOS gates are used as delay elements, may be used instead. These devices should be sufficiently compact to include on the discriminator board.

It should be possible to teat this approach using inexpenaive field-programmable gate array devices. 


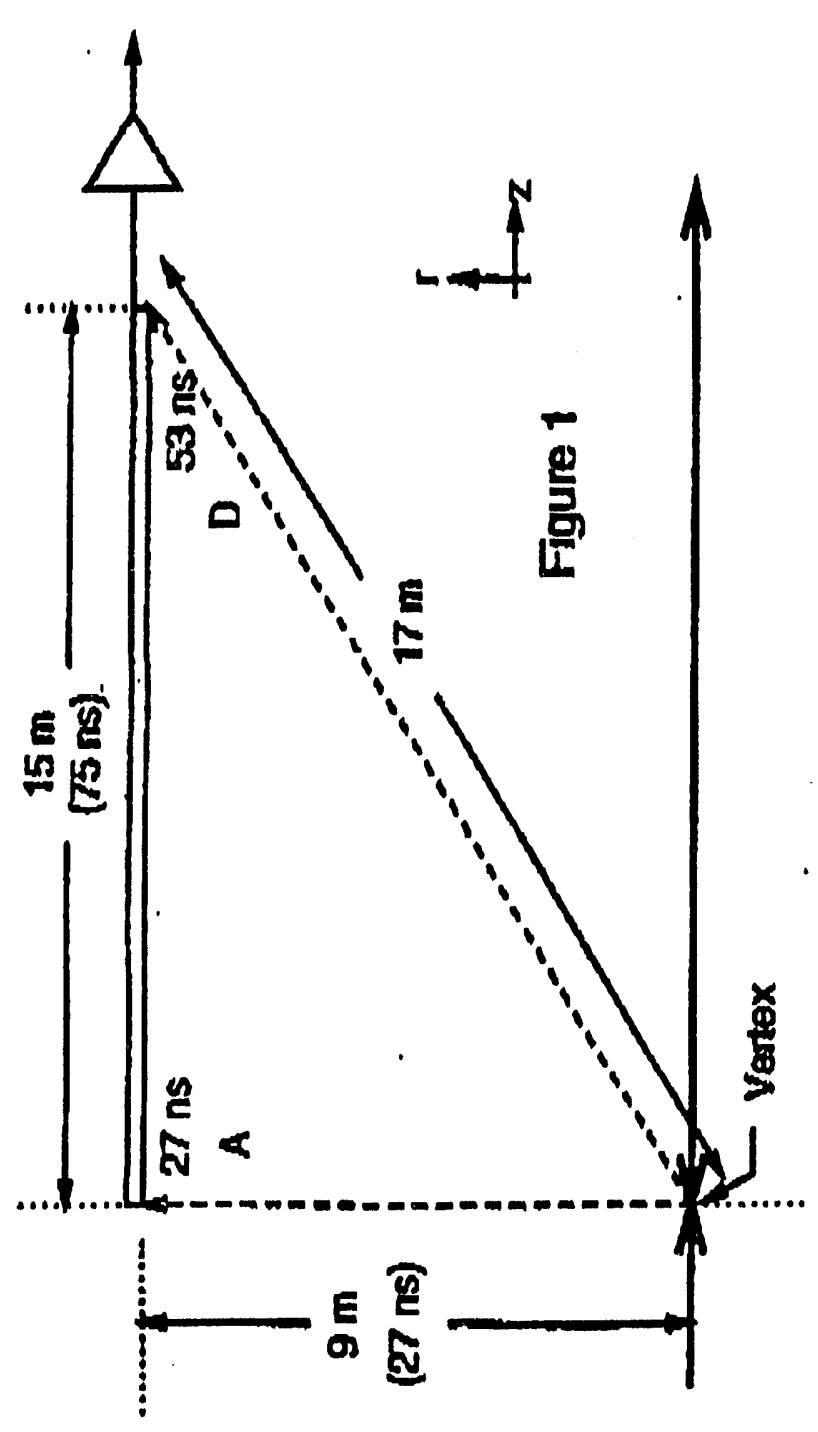

$09 / 30 \quad 16: 23$

$7312025 \# 44$ 


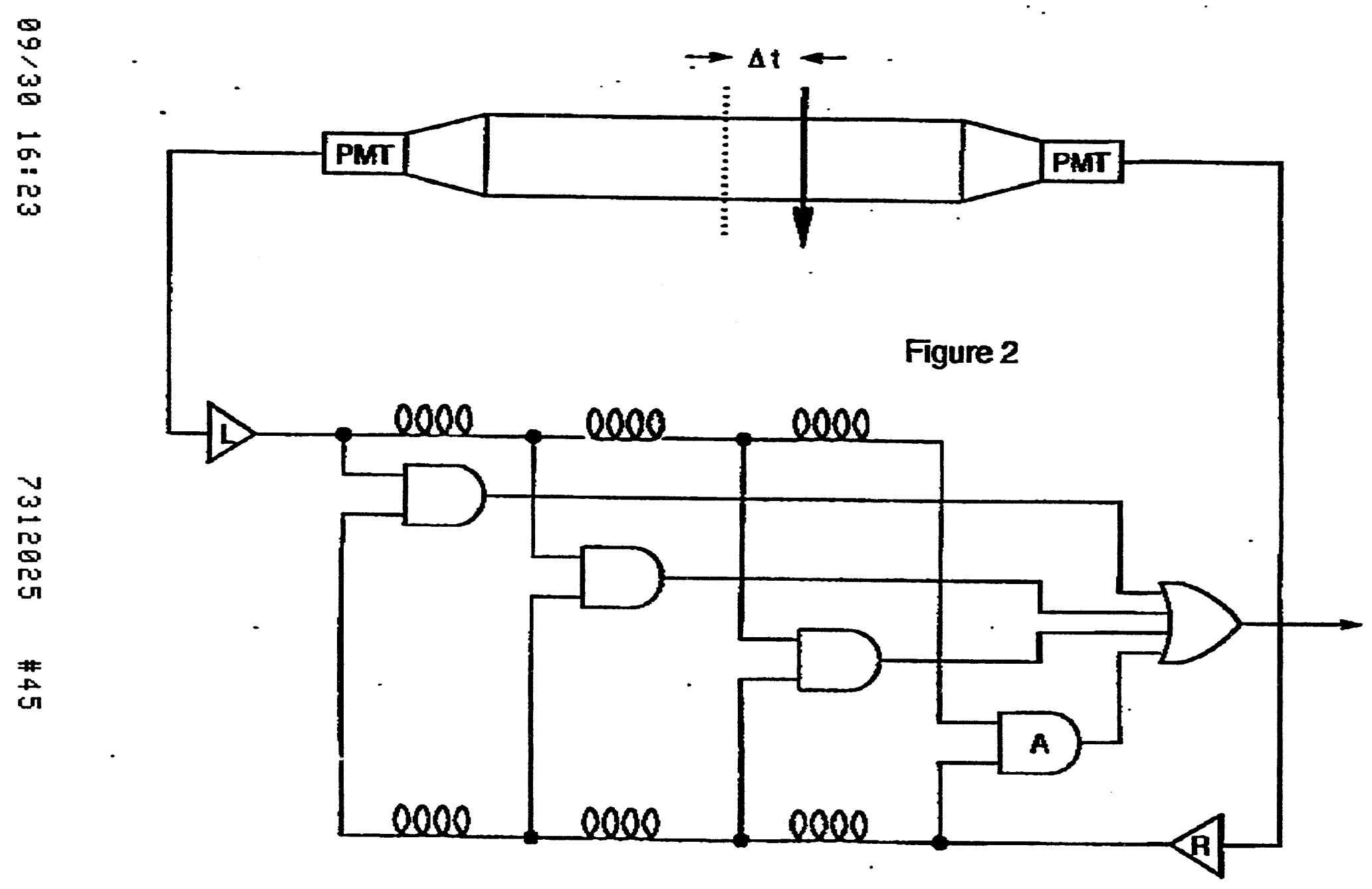




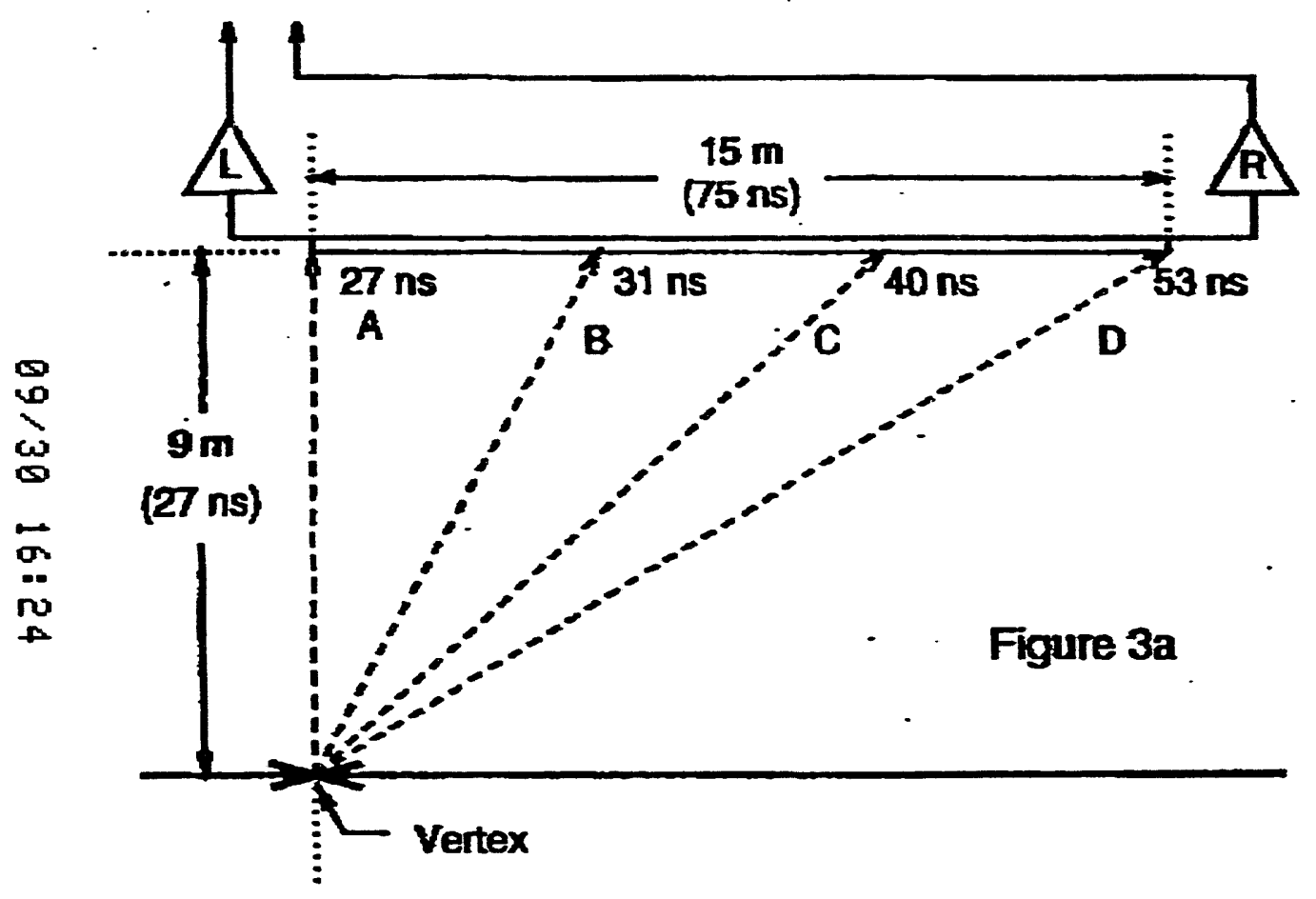

\begin{tabular}{|c|c|c|}
\hline \multicolumn{3}{|c|}{ Arrival Time in ns } \\
\hline Case & $T_{L}$ & $T_{R}$ \\
\hline A & 27 & 102 \\
\hline B & 56 & 81 \\
\hline C & 90 & 65 \\
\hline D & 128 & 53 \\
\hline
\end{tabular}

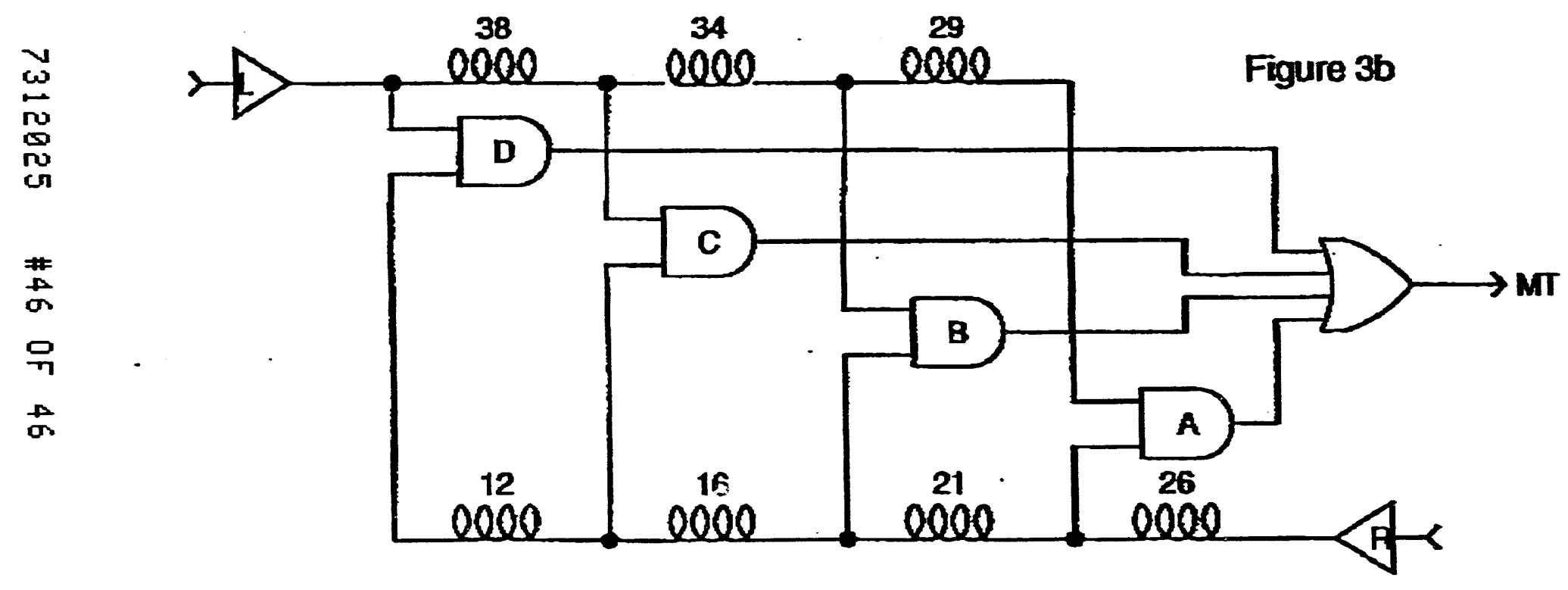


APPENDIX 3

Behavior of Large Resistive Plate Counters

Draft GEM Note

September, 1992 


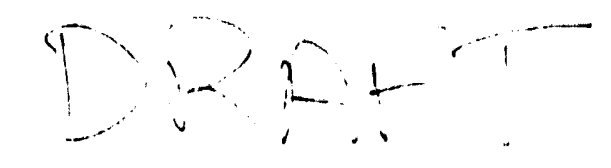

SSC GEM NOTE 1

$(92-1)$

\title{
BEHAVIOR OF LARGE RESISTIVE PLATE COUNTERS
}

SEPTEMBER 1992

\author{
M. Widgoff \\ Brown University \\ Providence, RI 02912 \\ E. D. Alyea \\ Indlana University \\ Bloomington, IN 47401 \\ E. Ables, R. Bionta, G. J. Mauger, C. Wuest \\ Lawrence Livermore National Laboratory \\ Livermore, CA 94550 \\ Y. H Chang, D. Chen, E. S. Hafen, P. Haridas, M. Lee, I. A. Pless, S. Yunus \\ Massachusetts Institute of Technology \\ Cambridge, MA 02139 \\ R. Santonico \\ University of Rome and Istituto Nazionale di Fisica Nucleare \\ Rome, Italy \\ S. Berridge, W. Bugg, P. Y. C. Du \\ University of Tennesseo \\ Knoxville, TN 37996
}


The performance of two Resistive Plate Counters, one made of Bakelite and the other out of two Wall Mirrors, is compared with a view to determine the operating characteristics as a function of the resistivity of the chamber wall material and the style of construction. We find that resistivities as high as $5 \times 10^{12} \Omega-\mathrm{cm}$ ( the Wall Mirror RPC) prevents the chamber from achleving adequate voltage in the gas gap, thereby limiting both chamber efficiency and time resolution. The time resolution of the Bakelite RPC (resistivity $\sim 10^{11} \Omega-\mathrm{cm}$ ) was measured to be $1.2 \mathrm{~ns}$, and efficiency over $95 \%$. Desplte the differences in the construction style, the pulse characteristics of the two RPCs are remarkably similar. Results regarding the sensitivity of the Bakelite RPC to low energy $(1-10 \mathrm{MeV})$ photons and neutrons are also provided. 


\section{INTRODUCTION}

Resistive Plate Counters (RPCs), are wireless, gaseous detectors that operate at a voltage sufficiently high to cause the fast formation of a streamer, when the gas is ionized by a charged particle traversing the chamber. The cential idea behind the principle of operation is to confine the discharge (spark) caused by the developing streamer to a localized region by using plates of high resistivity. In the last twenty years there has been considerable activity in the development of two different types of RPCs.

The first type of Resistive Plate Counters (Ref. 1) were small chambers $(10 \mathrm{~cm} \times 10 \mathrm{~cm})$ that operated at a high electric field $(E \sim 40 \mathrm{KV} / \mathrm{mm})$ and about 6 to 12 times atmospheric pressure. These counters are characterized by signal rise times well below 1 ns and time jitter of the order of 50 ps. However, the highly demanding construction criteria of these counters inhibited the production of large chambers of this type. The second type of RPC (Ref. 2, 3, 4), operate at lower electric fields $(E \approx 4 \mathrm{KV} / \mathrm{mm})$, and at atmospheric pressure. They provide signals with rise times of $2-3 \mathrm{~ns}$ and time jitter of the order of $1 \mathrm{~ns}$. It was also demonstrated that large RPCs of the order of $1 \mathrm{~m} \times 2 \mathrm{~m}$ could be constructed, and this development was quickly followed by its use in fixed target accelerator and cosmic ray experiments (Ref. 5). The general behavior of both types of counters is predictable in terms of standard parameters like the drift velocity and the Townsend's first coefficient for the gas mixture for any particular cholce of the electric field and gas pressure. The fast rise time and low jitter of these counters make them very appealing for use in fast trigger and time of flight applications. This study grew out of our interest in the use of RPCs for the muon trigger system in future collider detectors for SSC and LHC. In this note, we compare the performance of two large RPCs, one made of Bakelite (at the University of Rome) and the other made out of two Wall Mirrors (at MIT). This comparative study focuses on the dependence (if any) of RPC porformance pararneters like efflci $r \cdots r$, pulse rise time, time jitter and rate capability on the choice of chamber wall material as well as the style of construction. It is our opinion that comparative studies, such as this, is necessary to highlight those aspects of the construction of large RPCs that might require special care in its implementation.

In Section II we outline the details of construction of the two RPCs. In Section III we compare the performance of the Glass RPC against that of the more fully developed Bakelite RPC. Section IV contains a discussion of the sensitivity of RPCs to low energy $(1-10 \mathrm{MeV})$ photons and neutrons. 


\section{DETAILS OF RPC CONSTRUCTION}

The details of the construction of the Bakelite and Glass RPCs are compared in Table I and a cross sectional view of both RPCs is given in Fig. I $a, b$. An important parameter that controls RPC performance is the resistivity of the material that make up the chamber walls. The Bakelite RPC has a resistivity of $10^{11} \Omega-\mathrm{cm}$, while the Glass RPC resistivity was measured to be about $5 \times 10^{12} \Omega-\mathrm{cm}$. To achieve low noise and high efficiency, the Bakelite surfaces facing the gas were polished and painted with a linseed oil based semi-conducting paint (Ref. 1). The Glass RPC was built out of two mirrors $(1.35 \mathrm{~m} \times .4 \mathrm{~m})$ purchased from a local hardware store and so no care has been taken to control the glass composition or surface irregularities. The gas gap for both RPCs is $2 \mathrm{~mm}$ and the wall thickness differ by $1 \mathrm{~mm}$.

The high voltage and ground for the Bakelite RPC is provided by graphite paint on the outside surface of the Bakelite, to which the electrical connections are made. The high voltage and ground for the Mirror Glass RPC is provided by the metallic reflective coating of the mirrors, to which electrical connection was made using copper tape. It should be noted here, that these mirrors were not produced with the intention of using them for RPC construction and hence the electrical properties of the metallic coating was not finely controlled as in the Bakelite case. The signal readout for the Bakelite RPC is achieved by using aluminum strips, isolated from high voltage and ground by a thin insulating film. For the Glass RPC, the readout is achieved by segmenting the metallic coating on the ground side into strlps that run along the iength of the mirror. This was achieved by removing $\sim 1 \mathrm{~mm}$ of the reflective coating between adjacent strips, thus electrically isolating them from one another.

\section{PERFORMANCE CHARACTERISTICS}

In this section we discuss the results of various measurements performed on the Bakelite and Glass RPCs. We emphasize here that both RPCs were studled using the same experimental setup, i. e. the same gas system, the same high voltage supply, the same electronic components and in the case of efficiency and time fltter measurements, the same scintillator telescopes. A summary of the various measured parameters for the Bakelite and Glass RPCs is collected together in Table II. We discuss these results below.
$09 / 16 \quad 16: 38$
7312925
$\# 05$ 


\section{i) Pulse Characteristics}

The characteristics of the pulse from RPCs are quite well known. We find the pulse shape and rise time for the two RPCs agree remarkably well. To Illustrate this, we show in Fig. II $a, b$ the pulses from the Bakelite and Glass RPCs as observed on a LeCroy 9250 digitizing oscilloscope. In the case of the Glass RPC it was possible to lonize the gas mixture using a pulsed nitrogen laser. Fig. II $c$ shows a laser induced pulse from the glass RPC. Since the pulsing frequency of the nitrogen laser can be varied, the laser is a very effective way to examine the local recovery time of the RPC. The laser beam can inject pulses into the same local region and the recovery time directly measured. We observed that the recovery time for the Glass RPC was about one second.

\section{ii) Esticiency}

Efficiency measurements using cosmic rays and a scintillation counter telescope for trigger, yields about $95 \%$ efficiency for the Bakelite RPC and $70 \%$ efficiency for the Glass (mirror) RPC. The results of efficiency measurements for various discriminator threshold values from 16 millivolts to 1000 millivolts are shown in Fig. III a for the Bakelite RPC and in Fig. III $b$ for the Glass RPC. A threshold of 100 millivolts was chosen as a good operational value for the Bakelite RPC. No measurements were made for the Bakelite RPC for high voltage greater than $9.5 \mathrm{KV}$, because of the onset uf nolse.

Current measurements were made for the two RPCs as a function of the supply high voltage. Results of these measurements are given in Fig. IV a, b. While the Glass RPC draws upto 5 microamps for the voltage region under study, the Bakelite RPC draws 50 microamps. To investigate the lack of full efficiency for the Glass RPC we calculated the effective voltage across the gas gap, using the measured current value and the resistance of the chamber wall material as input. $V(\theta f f)$ is here defined as $V(e f f)=[V(s)-2(D V)]$, where $V(s)$ is the supply voltage to the RPC, and DV is the voltage drop across one chamber wall. Fig. $V$ a, b shows $V(e f f)$ as a function of the supplied voltage, $V(s)$. For the Bakelite RPC we find a linear increase for a resistivity of $10^{11} \Omega-\mathrm{cm}$, with a negligible difference between the effective and supply voltage. For a resistivity of $10^{12} \Omega-\mathrm{cm}$, the calculated effective voltage deviates from the supplied voltage by increasing amourits as supply voltage is increased. At a supply voltage of $9.5 \mathrm{KV}$ the difference between the supplied and effectlve voltage is about $1 \mathrm{KV}$. The two values of the resistlvity used here are the limiting values for the Bakelite RPC. 
The effective voltage for the Glass RPC shows a quite different behavior. After a steady increase from $V(s)=6 \mathrm{KV}$ to $\mathrm{V}(\mathrm{s})=7 \mathrm{KV}$, the value of $V$ (eff) dips a smaller amount and then proceeds to have a very slow growth. At $V(s)=10 \mathrm{KV}, \mathrm{V}(e \mathrm{ff})=6.9 \mathrm{KV}$. The measured efficiency curve for the Glass RPC (Fig. II b) shows a behavior quite compatible with the behavior of $V$ (eff). This suggest that the flattening off, of the efficiency at $70 \%$ is due to a lack of adequate growth of voltage across the gas gap. This result suggests that it is impractical to use materials of resistivity greater than $10^{12} \Omega$-cm for RPCs, because of the limitation it imposes on chamber efflciency.

\section{iii) Counting Rate}

The counting rate of a single strip ( $3 \mathrm{~cm}$ wide) as a function of the threshold setting of the discriminator is shown in Fig. VI a, b. Data were taken at three different high voltage values, 7,8 and $9 \mathrm{KV}$. The strip length is $2 \mathrm{~m}$ for the Bakelite RPC and $1.35 \mathrm{~m}$ for the Glass RPC.

The data from both RPCs show an increase in the counting rate as the voltage is increased. Since the efficiency of the RPCs (see Fig. III $a, b$ ) is essentially flat in the high voltage region from 7 to $9 \mathrm{KV}$, it is clear that this increase in the counting rate is indicative of a growth of noise of the system as the high voltage is increased. Furthermore, the counting rate shows a steady decrease for threshold values greater than $100 \mathrm{mv}$ and steep drop from $16 \mathrm{mv}$ to $100 \mathrm{mv}$. This suggests that the intrinsic noise is constituted of pulses with varying pulse height, the larger fraction of which have pulse heights less than $100 \mathrm{mv}$ and a smaller fraction with pulse heights greater than $100 \mathrm{mv}$. It is important to emphasize that although a large percentage of the noise can be filtered out from the readout electronic modules by appropriate choice of the discriminator threshold setting, they could lower the efficiency of RPCs with high volume resistivity. This can be seen from the Glass RPC results, where the efficiency shows about $10 \%$ to $15 \%$ decrease in the region of 8 to $9 \mathrm{KV}$.

The counting rate per unit area $\left(\mathrm{cm}^{2}\right)$ for both RPCs is given in Table II, for two different thresnold values of the discriminator. For both RPCs the counting rate at $16 \mathrm{mv}$ is twice as large as the value at $100 \mathrm{mv}$. To compare the response of the two RPCs to a strong radioactive source we measured the counting rate for a localized region using a 1 Millicurie Strontium -90 medical source.

The source was placed at the center of a strip, and the irradiated area was defined as the area of the front face of the source through which the

$$
09 / 1616: 39 \quad 7312025 \quad \# 07
$$


radiation exited. A copper collimator was slipped onto the cylindrical barrel containing the source, so as to prevent radiation form exiting through the sides. The irradiated area was $1.33 \mathrm{~cm}^{2}$. Using this arrangement, we found the Bakelite RPC to have a counting rate of $543 \mathrm{~Hz} / \mathrm{sqcm}$ and the Glass (mirror) RPC, $48 \mathrm{~Hz} / \mathrm{sqcm}$. From the resistivity of the materials $\left(2 \times 10^{11} \Omega\right.$-cm for bakelite and $5 \times 10^{12}$ for glass) and the discharge area of the spark $0.1 \mathrm{~cm}^{2}$ for bakelite (Ref. 2) and $0.4 \mathrm{sqcm}$ for glass (Ref. 6) we calculate the "saturating counting rates" to be $561 \mathrm{~Hz}$ / sqcm for Bakelite RPC and $41 \mathrm{~Hz} / \mathrm{sqcm}$ for Glass RPC. (At the "saturating couniting rate" the localized region is dead for particle detection). For a beam intensity of $100 \mathrm{~Hz} / \mathrm{cm}^{2}$ the above result for the Bakelite RPC would imply a $20 \%$ loss of efficiency. For a resistivity of $1 \times 10^{11} \Omega-\mathrm{cm}$, the loss in efficiency would be $10 \%$.

\section{iv) Iime Resolution [vitter]}

Several measurements of the time resolution were made for both RPCs at different locations of the chamber. The measurements were made with a scintillator telescope arrangement and a LeCroy $2228 \mathrm{~A}$ TDC. The best measured resolutions are shown in Fig. VII $a, b$. The Bakelite RPC has a resolution of $1.25 \mathrm{~ns}$ at $8 \mathrm{KV}$, while the glass RPC resolution is at about 7 ns.

Fig. VIII $a, b$ shows the behavior of the time resolution measurements as a function of the chamber high voltage. For the Bakelite RPC (Fig. VIII a), the time resolution exhibits a characteristic fall off, reaching a minimum at $8 \mathrm{KV}$ and then rising to a slightly higher value at 9 KV. The Glass RPC time resolution on the other hand shows a flat behavior, and this is to be expected, because, as demonstrated earller the effective voltage variation in the gas gap for this RPC is also almost flat.

\section{RPC SENSITIVITY TO LOW ENERGY PHOTONS AND NEUTRONS}

The sensitivity of RPC to low energy photons and neutrons is an important consideration when these chambers are operated in high radiation environment like the SSC or LHC. A high sensitivity to this type of radiation would not only degrade the RPC efficiency for muons, but could also glve rise to accidental triggers. An investigation of the performance of RPC in a high radiation was carried out (Ref. 7) by measuring the chamber efficiency in a beam emerging from a nuclear reactor. However, the neutron component of the beam wis shielded out by water, and further more the experiment did not provide an estimate of the
$09 / 1616: 40$
$7312025 \quad \# 08$ 
sensitivity of the chamber to photons.

To get an estimate of the sensitivity of the Bakelite RPC to low energy photons and neutrons we exposed a limited region $\left(135 \mathrm{~cm}^{2}\right)$ of the sensitive area of the RPC to a strong Cf-25? source. Knowing the effective mass of the source, and the geomeiry of the experimental setup, we were able to determine the total flux of photons and neutrons passing through the RPC. We employed shielding techniques to isolate the photon and neutron components.

Fig. IX shows the attenuation in the counting rate for various shield combinations interposed between the Cf-252 source and the RPC. The counting rate drops from no shield value of $9800 \mathrm{~Hz}$ to $1500 \mathrm{~Hz}$, when 6.2 $\mathrm{cm}$ of lead is placed between source and detector. Counting rates were also measured for two additional polyethylene slabs added one at a time to the shield. It is well known that when polyethylene is used as a neutron shield, neutron capture in polyethylene leads to gamma emission. In order to make this explicit, we have labelled the curve obtained as a result of the shielding as $\left(n+\gamma_{p}+\gamma_{s}\right)$ where $n$ stands for neutron, $\gamma_{p}$ for the primary gammas from the source, arid $\gamma_{s}$ for those secondary gammas generated in the polyethylene shield. The secondary gammas enter the detector and thus masks the behavior expected from neutron attenuation alone. By subtracting off the $\gamma_{p}$ component, we obtain the second curve below the first one. This curve when extrapolated to the no shield value, gives the number of neutron interactions in the detector. The remaining inieractions are thus attributable to photons. From the source strength (62 microcuries) and the geometry of the experimental setup we find the total flux entering the detector to be:

$$
\begin{aligned}
& N_{\gamma}=2.0 \times 10^{6} / \mathrm{s} \\
& N_{n}=1.2 \times 10^{5} / \mathrm{s}
\end{aligned}
$$

Using the number of $n$ and gamma interactions in the chamber, and the total flux through it, we obtain the neutron and photon sensitivity given in Table III. Also given in Table III is the calculated value of the expected interaction probability for the gas alone. For neutrons, the sensitivity of the gas alone is an order of magnitude less than the measured value. It is clear that the above discrepancy can be accounted for by the interaction of neutrons in the walls of the chamber. This suggests that a lower sensitivity could be achieved by appropriate choice of material that have low cross section for neutron interactions. However, gas contaminates

$$
99 / 16 \quad 16: 41
$$


(mostly hydrocarbons) would eventually precipitate out onto the walls and thereby negate the possible gain of using special materials.

\section{SUMMARY}

We have examined the behavior of two large RPCs, one made of bakelite (resistivity $=1$ to $2 \times 10^{11} \Omega$-cm) and the other of ordinary mirror glass (resistivity $=5 \times 10^{12} \Omega-\mathrm{cm}$ ).

We find that the high resistivity of the Glass RPC not only degrades the rate handilng capability, but also prevents the chamber form achieving full efficiency. On the other hand, the two RPCs agree remarkably well in terms of pulse characteristics, like pulse helght, pulse width, rise time and pules velocity along strip. In addition the readout technique employed in the Glass RPC suggests the possibility of creating readout strips by cutting out (for electrical isolation) strips from a uniform deposit of conductive material on the outside of the chamber walls. We obtain a time resolution of the order of $1 \mathrm{~ns}$ for the Bakelite RPC. The failure of the Glass RPC to achleve a time resolution comparable to the bakelite one can be easily explained by the failure of this RPC to achieve adequate high voltage across the gas gap. By using a strong Cf-252 source, we estimate the sensitivity of the RPC to $\operatorname{MeV}(1-10)$ neutrons to be $4.8 \times 10^{-3}$ and to $\mathrm{MeV}(1-10)$ photons to be $6.6 \times 10^{-3}$. It has been demonstrated (Ref. 8 ) that Bakelite RPCs can handle rates upto $100 \mathrm{~Hz} / \mathrm{cm}^{2}$, without significant loss of efficiency. We are currently engaged in the exploration of various techniques to enhance the rate capability by at least another order of magnitude. 


\section{REFERENCES}

1. W. Braunschweig, Physcis Scripta Vol, 23, $384-392,1981$. (This reference contains a summary of the efforts of many groups who studied these chambers).

2. R. Santonico et al., NIM 187 (1981), 377 - 380.

R. Cardarelli ot al., NIM A263 (1988), $20-25$.

3. G. Battistoni et al., NIM A270 (1988), $190-193$.

4. M. Anelli ot al., NIM A300 (1991), $572-574$.

5. G. Bressi et al., NIM A261 (1987), $449-461$.

M. Bertino ot al., NIM A283 (1989), 654 - 657.

M. Ambrossio et al., "Status Report on the MINI Experiment," International Workshop on Neutrino Telescopes, Venice, 1989.

6. G. Bencivenni et al. LNGS - 92/38, July 1992.

7. M. lori et al., NIM A306 (1991), $159-168$.

8. A. Bohrer et al., "Status of the RD5 Experiment," CERN/DRDC/91 - 53, January 1992. 


\section{FIGURE CAPTIONS}

Figure I Cross-sectlon view of (a) Bakelite RPC and (b) the Wall Mirror (Glass) RPC.

Figure II Cosmic ray induced pulses in (a) Bakelite RPC and (b) the Glass RPC as observed on a LeCroy 9250 digitizing oscilloscope. (c) Laser (Nitrogen) induced pulse in the Glass RPC.

Figure III Chamber efficiency as a function of high voltage for various discriminator threshold settings indicated in the figure.

(a) Bakelite RPC (b) Glass RPC.

Figure IV Current drawn by (a) Bakelite and (b) Glass RPCs at various values of the chamber high voltage.

Figure $V \quad T h e$ effective voltage in the gas gap as a function of voltage supplied to the chamber. $V$ (effective) $=V$ (supplied) $-2 D V$, where $D V=I R$, Is the voltage drop across each resistive plate. $V$ (effective) is displayed for two different limiting volume resistivity values of (a) Bakelite RPC and the measured resistivity of $5 \times 10^{12} \Omega-\mathrm{cm}$ for the Glass RPC.

Figure VI RPC single strip ( $3 \mathrm{~cm}$ wide) counting rate as a function of discriminator threshold setting for three different high voltage values. Area of the strip is (a) $600 \mathrm{~cm}^{2}$ for Bakelite RPC and (b) $400 \mathrm{~cm}^{2}$ for the Glass RPC.

Figure VII Distribution of the relative delay between the RPC and a scintillation counter cosmic ray telescope. (a) Bakelite (b) Glass RPC.

Flgure VIII Time resolution as a function of the chamber high voltage.

(a) Bakelite RPC and (b) Glass RPC. The high constant value of the Glass RPC time resolution is due to a lack of growth of the voltage in the gas gap.

Figure IX Counting rate as a function of effective interaction length of material inserted between source and RPC. In labelling the curves, $n$ refers to neutrons, $\gamma_{p}$ refers to primary (direct) 
photons from the source, and $\gamma_{S}$ refers to photons generated by neutron capture in the shielding material. 
DETAILSOF CONSTRUCTION

\begin{tabular}{|c|c|c|}
\hline PARAMETERS & BAKELITE RPC & GLASS (MIRROR) RPC \\
\hline MATERIAL OF CHAMBER & $\begin{array}{c}\text { BAKELITE } \\
\text { (Phenolic Polymer) }\end{array}$ & GLASS \\
\hline SIZE & $2 m \times 1 m$ & $1.35 \mathrm{~m} \times 0.4 \mathrm{~m}$ \\
\hline WAL THICKNESS & $2 \mathrm{~mm}$ & $3 \mathrm{~mm}$ \\
\hline VOLUME RESISTIVITY & $10^{11} \Omega \cdot \mathrm{cm}$ & $5 \times 10^{12} \Omega \cdot \mathrm{cm}$ \\
\hline GASGAP & $2 \mathrm{~mm}$ & $2 \mathrm{~mm}$ \\
\hline INTERNAL SPACERS & YES & NO \\
\hline $\begin{array}{l}\text { HIGHVOLTAGE SUPPLY } \\
\text { SURFACE }\end{array}$ & $\begin{array}{l}\text { GRAPHITE PAINT ON } \\
\text { BAKELITE }\end{array}$ & $\begin{array}{c}\text { REFLECTING SURFACEOF } \\
\text { MIRROR }\end{array}$ \\
\hline READOUT STRIPS & ALUMINUM & $\begin{array}{l}\text { STRIPS CUT OUTON } \\
\text { REFLETING SURFACE }\end{array}$ \\
\hline STRIP IMPEDANCE & $50 \mathrm{OHM}$ & $50 \mathrm{OHM}$ \\
\hline STRIP WIDTH & $3 \mathrm{~cm}$ & $3 \mathrm{~cm}$ \\
\hline GAS COMPOSITION & $\begin{array}{l}64 \% \text { ARGON, } 4 \% \text { F13B1 } \\
30 \% \text { ISOBUTANE }\end{array}$ & $\begin{array}{l}65 \% \text { ARGON, } 4 \% \text { F13B1 } \\
30 \% \text { ISOBUTANE }\end{array}$ \\
\hline
\end{tabular}

Table I 


\section{RERFORMANCE CHARACTERISTICS}

\begin{tabular}{|c|c|c|}
\hline PARAMETERS & BAKELITE RPC & GLASS (MIRROR) RPC \\
\hline PULSE HEIGHT & $0.3 \cdot 0.6$ VOLTS & $0.2-0.5$ VOLTS \\
\hline PULSE WIDTH & $\sim 10 \mathrm{~ns}$ & $-20 n s$ \\
\hline PULSE RISE TIME & $\sim 3 \mathrm{~ns}$ & $\sim 3$ ns \\
\hline $\begin{array}{l}\text { PULSE VELOCTTY } \\
\text { along strip }\end{array}$ & $>0.5$ ( $<6.6 \mathrm{~ns} /$ meter $)$ & $>0.5(<6.6 \mathrm{~ns} /$ meter $)$ \\
\hline EFFICIENCY & $>95 \%$ & $-70 \%$ \\
\hline PLATEAU KNEE & $\sim 7$ KVOLTS & $\sim 7 \mathrm{KVOLTS}$ \\
\hline PLATEAU WIDTH & $\sim 2000$ VOLTS & $\sim 2000$ VOLTS \\
\hline CURRENT DRAWN & $\sim 25$ microAmp @ $8 \mathrm{KV}$ & $\sim 2$ microAmp @ $8 \mathrm{KV}$ \\
\hline $\begin{array}{l}\text { COUNTING RATE } \\
\text { al } 8 \text { Kvolls } \\
\text { (@ two thresholds) }\end{array}$ & $\begin{array}{l}0.3 \mathrm{~Hz} / \mathrm{sqcm} @ 0.016 \mathrm{v} \\
0.1 \mathrm{~Hz} / \mathrm{sqcm} @ 0.100 \mathrm{v}\end{array}$ & $\begin{array}{l}0.2 \mathrm{~Hz} / \mathrm{sqcm} @ 0.032 \mathrm{v} \\
0.1 \mathrm{~Hz} / \mathrm{sqcm} @ 0.100 \mathrm{v}\end{array}$ \\
\hline $\begin{array}{l}\text { SOURCETEST } \\
\text { RATE / CM } \\
\text { ("medical source") }\end{array}$ & - $543 \mathrm{~Hz} / \mathrm{sqcm} @ 0.016 \mathrm{v}$ & $\sim 48 \mathrm{~Hz} / \mathrm{sqcm} @ 0.016 \mathrm{v}$ \\
\hline $\begin{array}{l}\text { TIME RESOLUTION } \\
\text { (JITTER) }\end{array}$ & $-1.1 \mathrm{~ns}$ & $-7 n s$ \\
\hline
\end{tabular}

Table II 
RPC SENSITIVITY TO LOW ENERGY ( 1 - 10 MeV) PHOTONS \& NEUTRONS

\begin{tabular}{|c|c|}
\hline PARAMETER & BAKELITE RPC \\
\hline PHOTON SENSITIVITY & $6.6 \times 10^{-3} / \mathrm{PHOTON}$ \\
\hline NEUTRON SENSITIVITY & $4.5 \times 10^{-3} /$ NEUTRON \\
\hline $\begin{array}{l}\text { NEUTRON SENSITIVITY } \\
\text { in Gas Alane }\end{array}$ & $1 \times 10^{-4} /$ NEUTRON \\
\hline
\end{tabular}

Table III 
Bakelle RPC

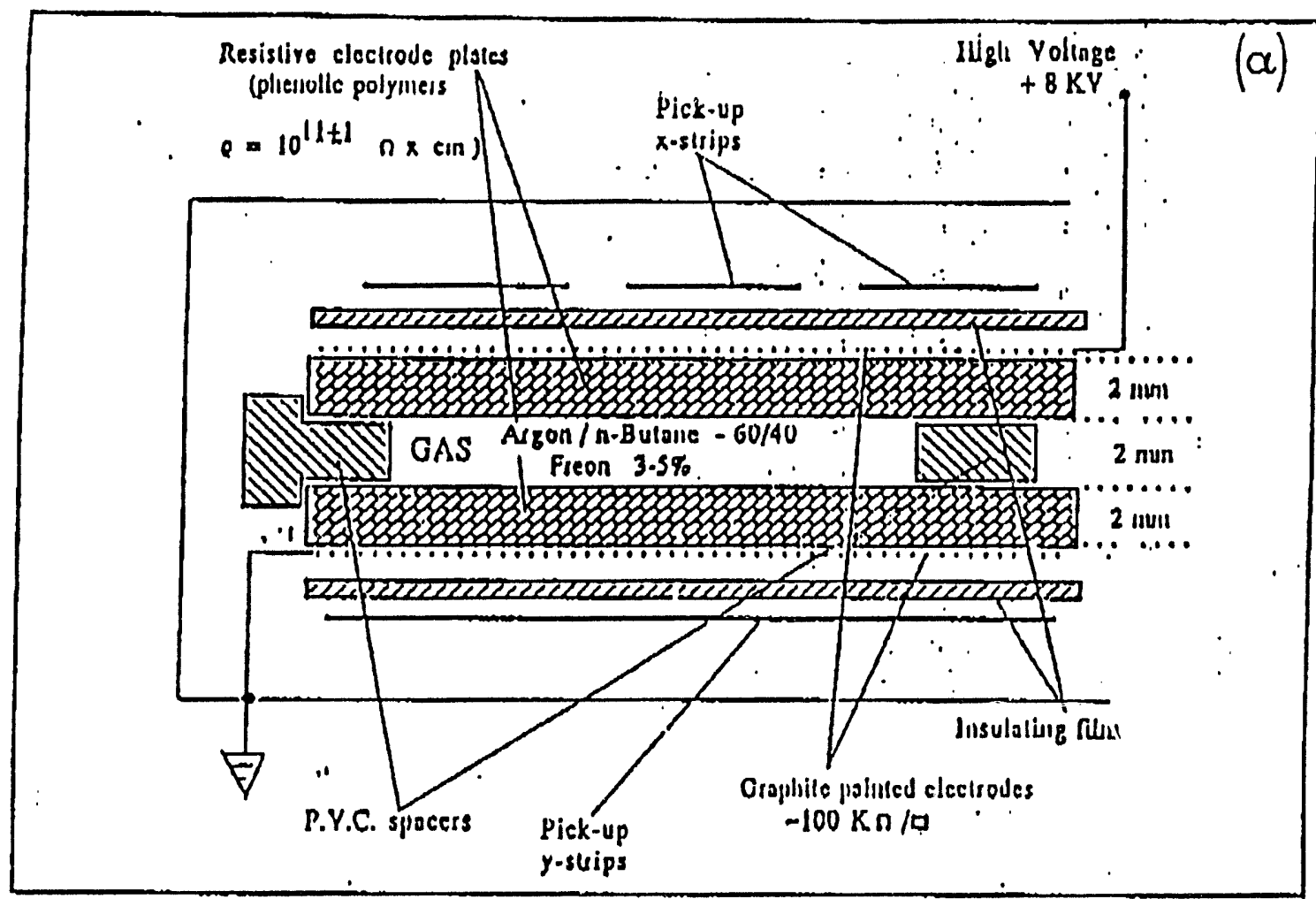

Glass APC

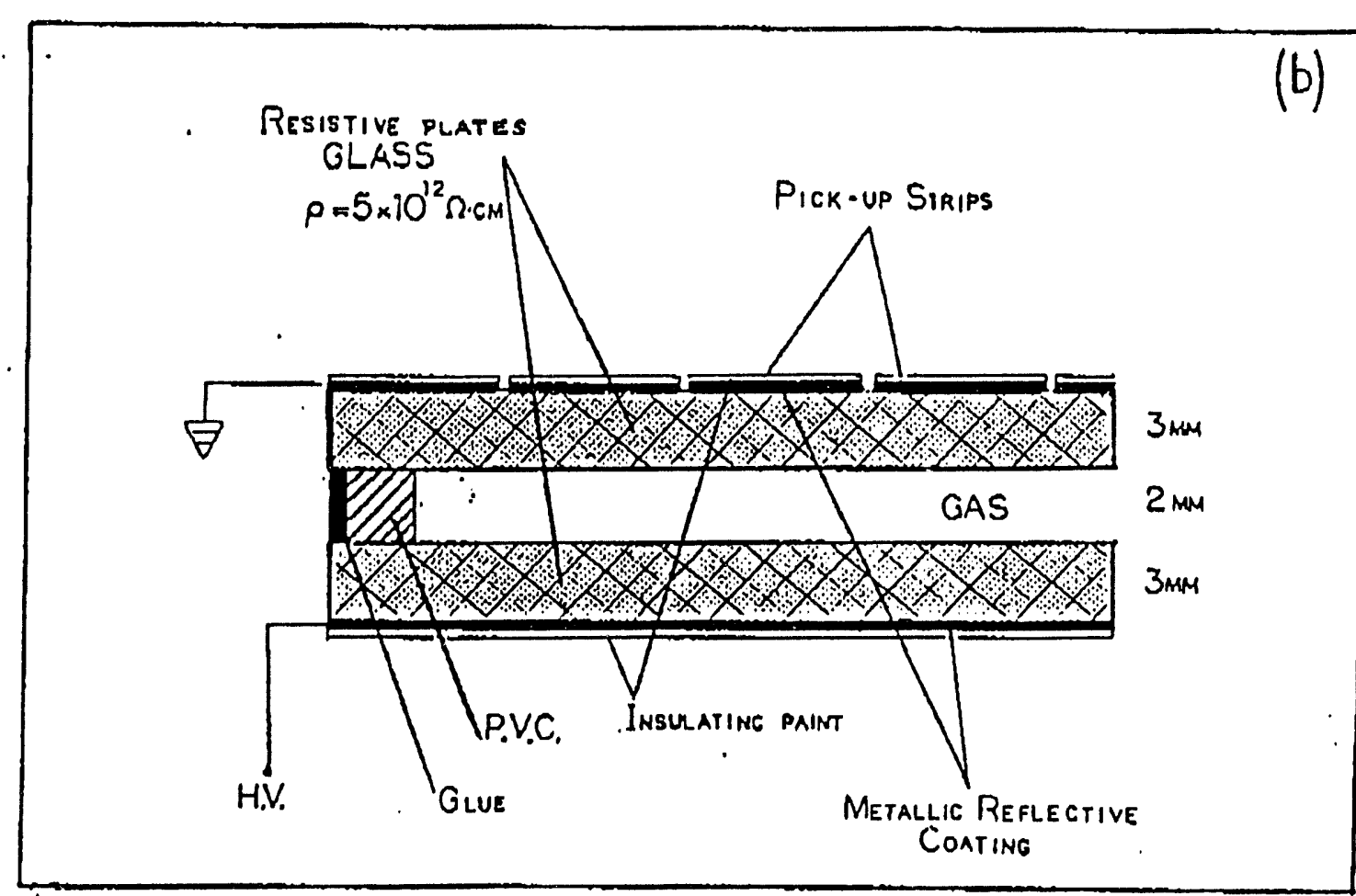

FIG.I 

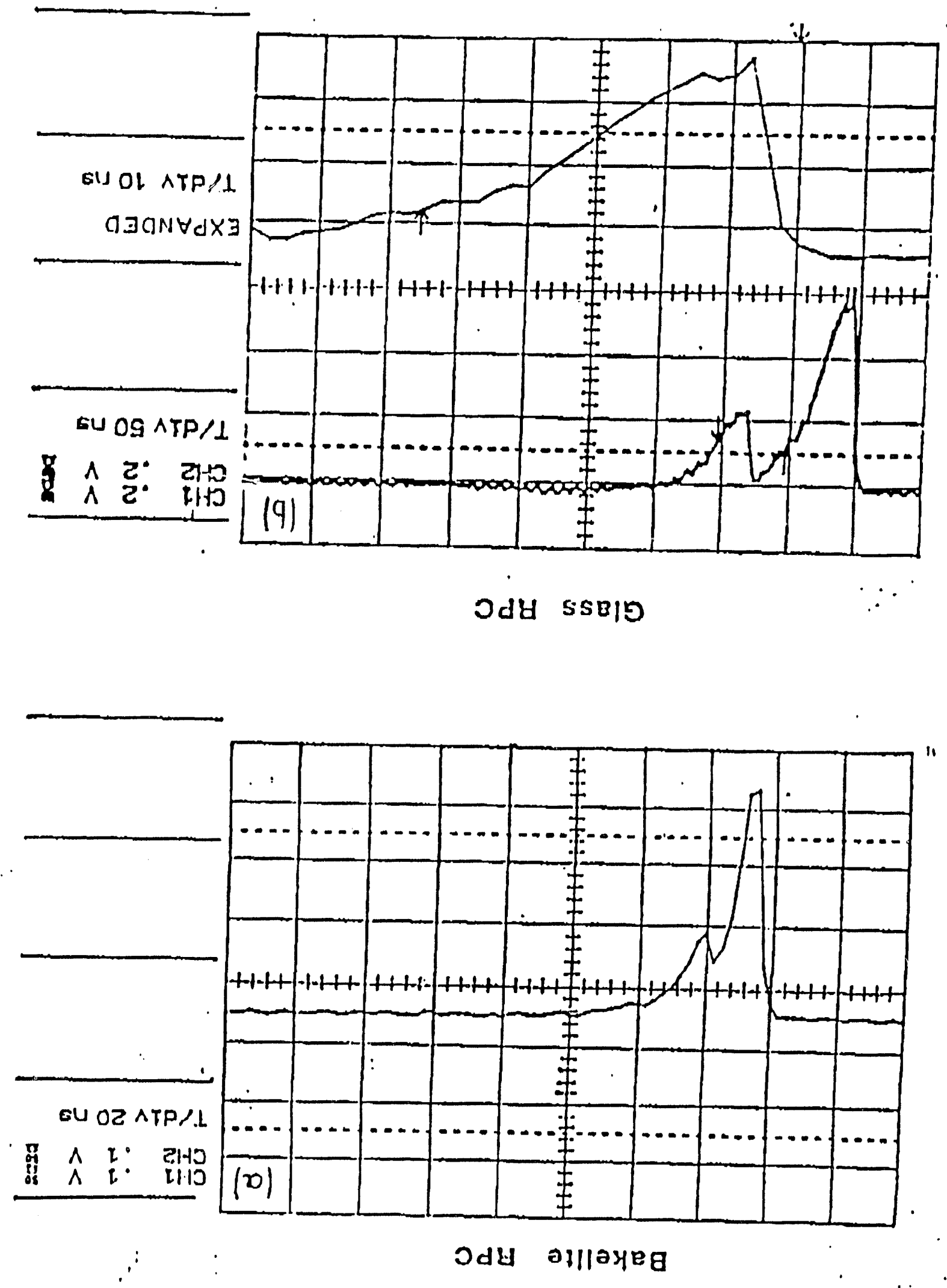


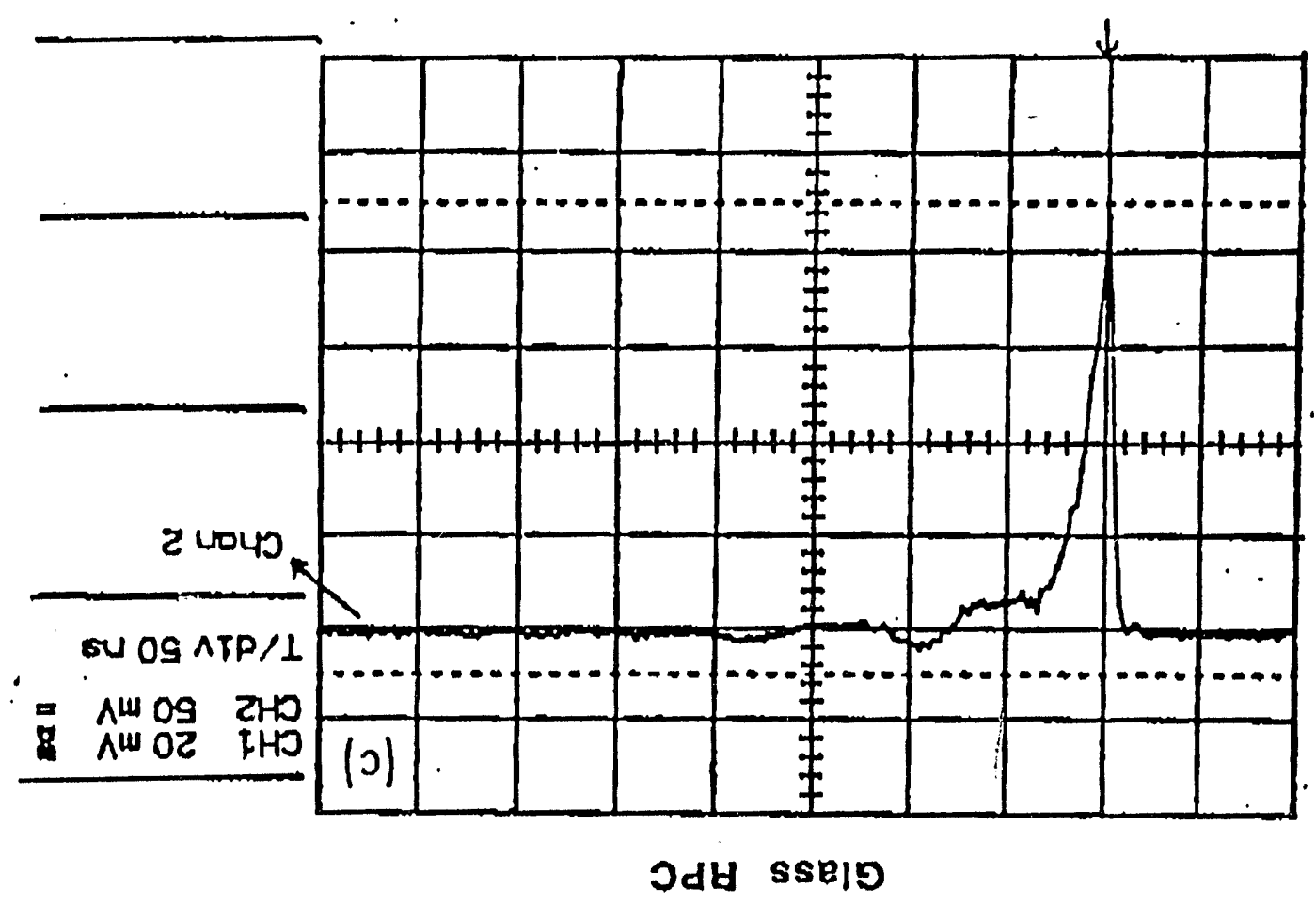



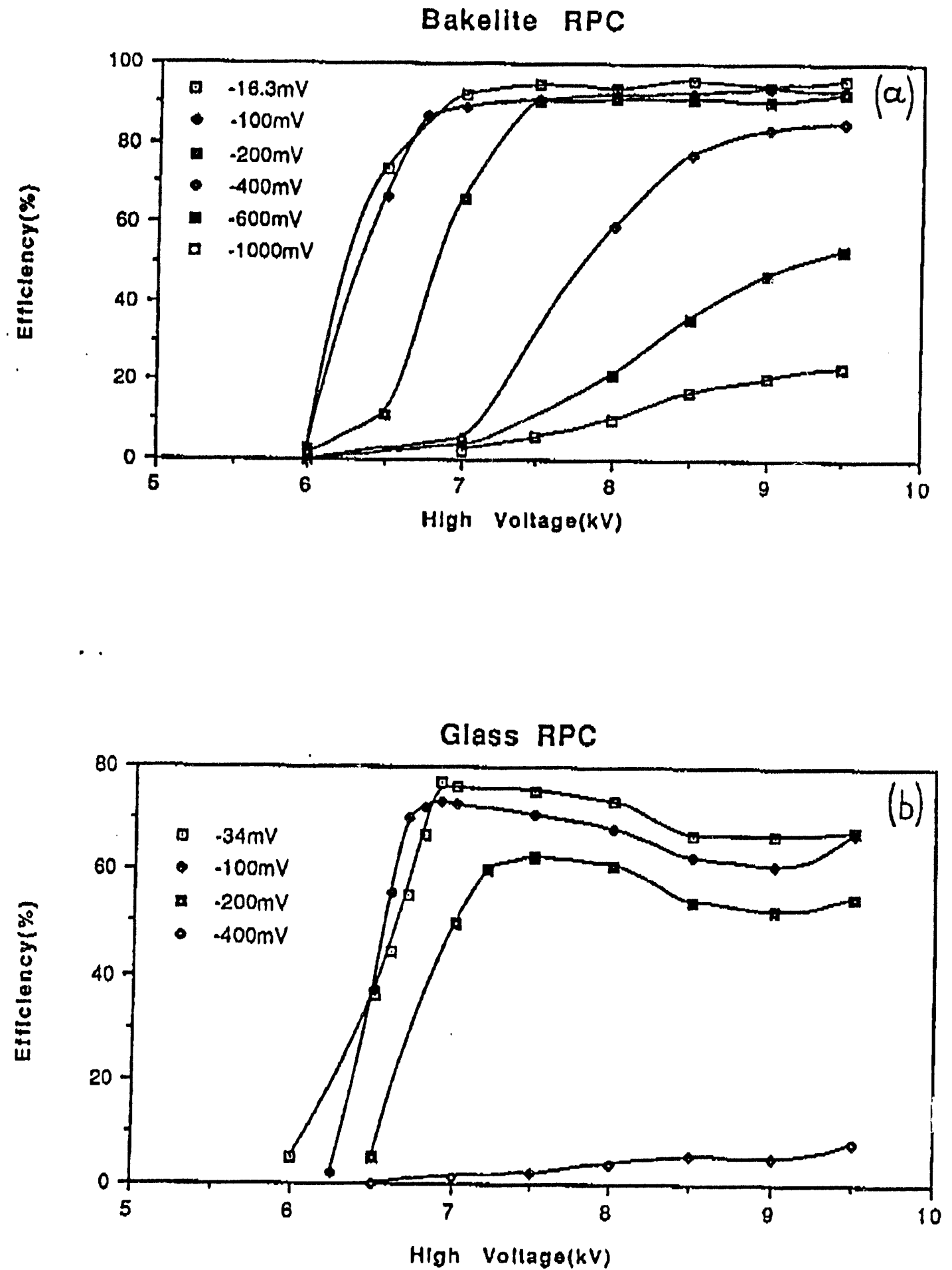

Fig.III 

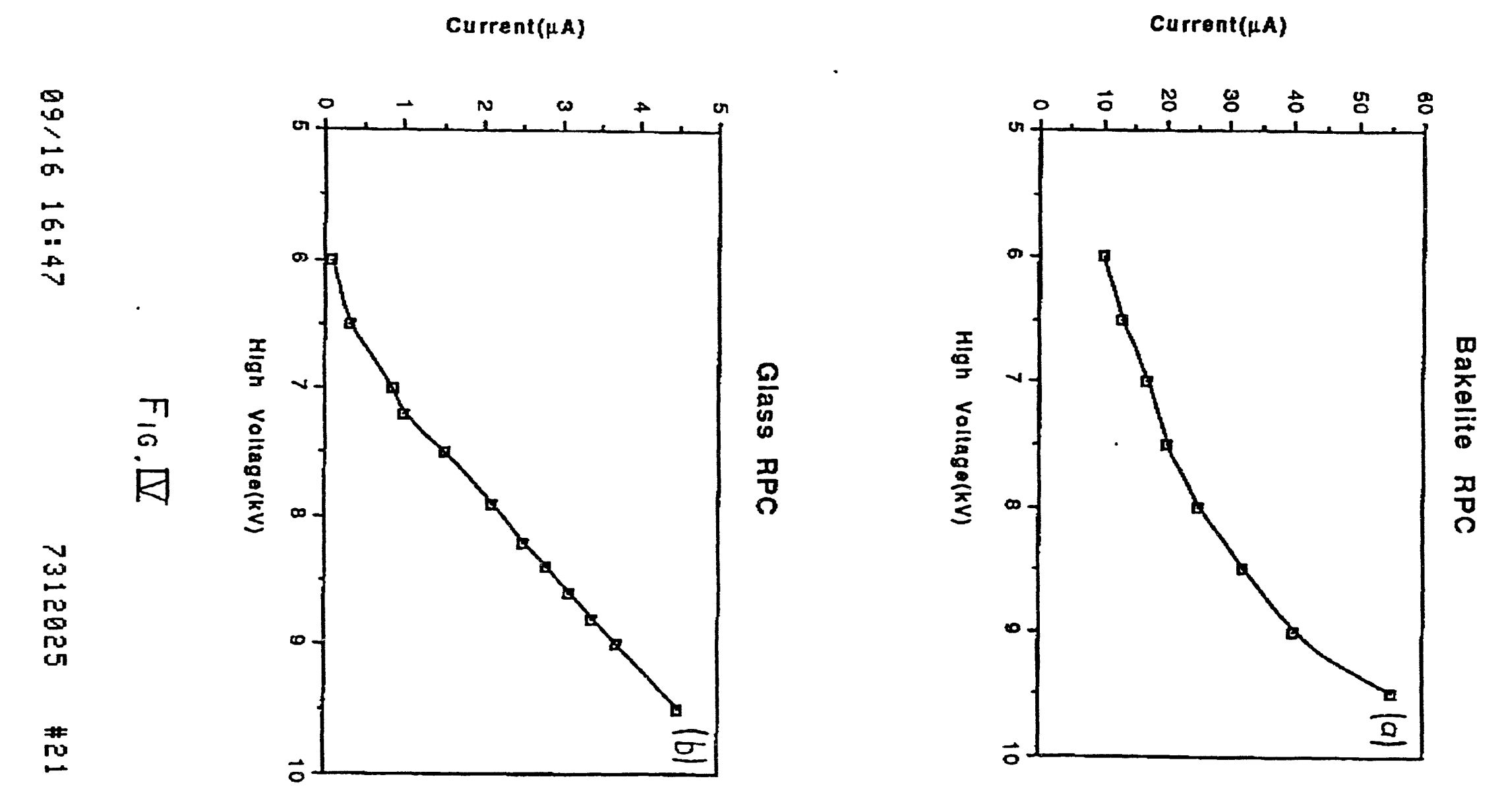

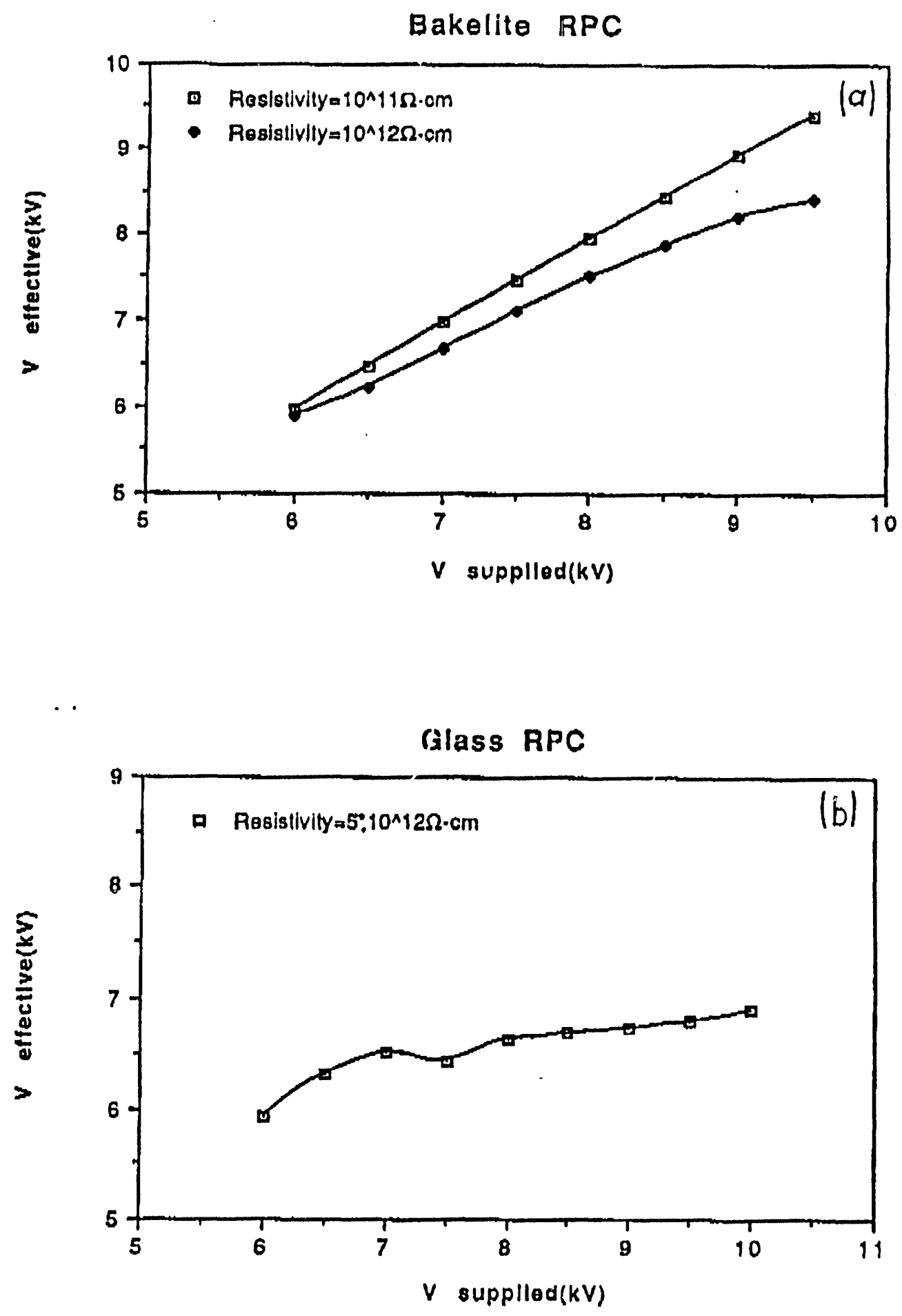

FIG.Z 


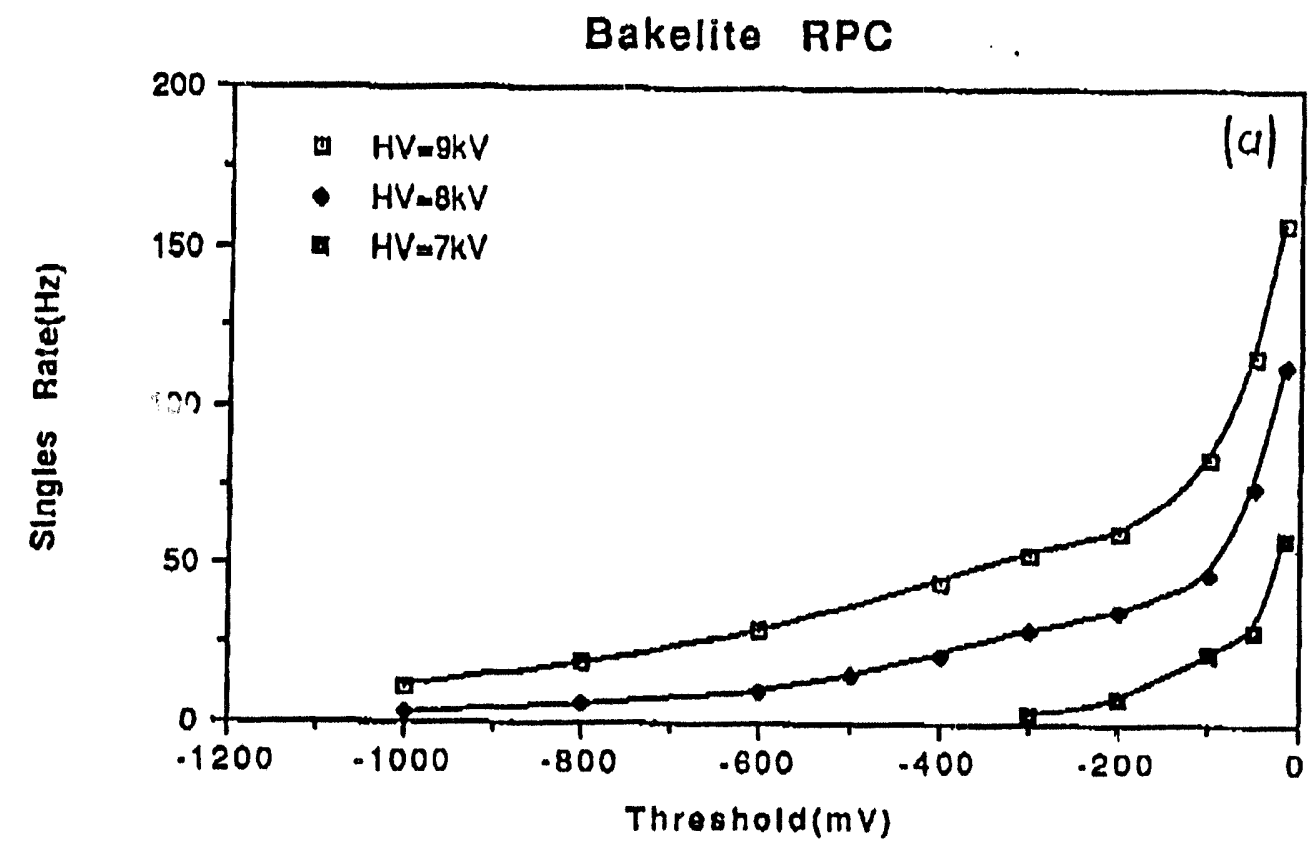

Glass RPC

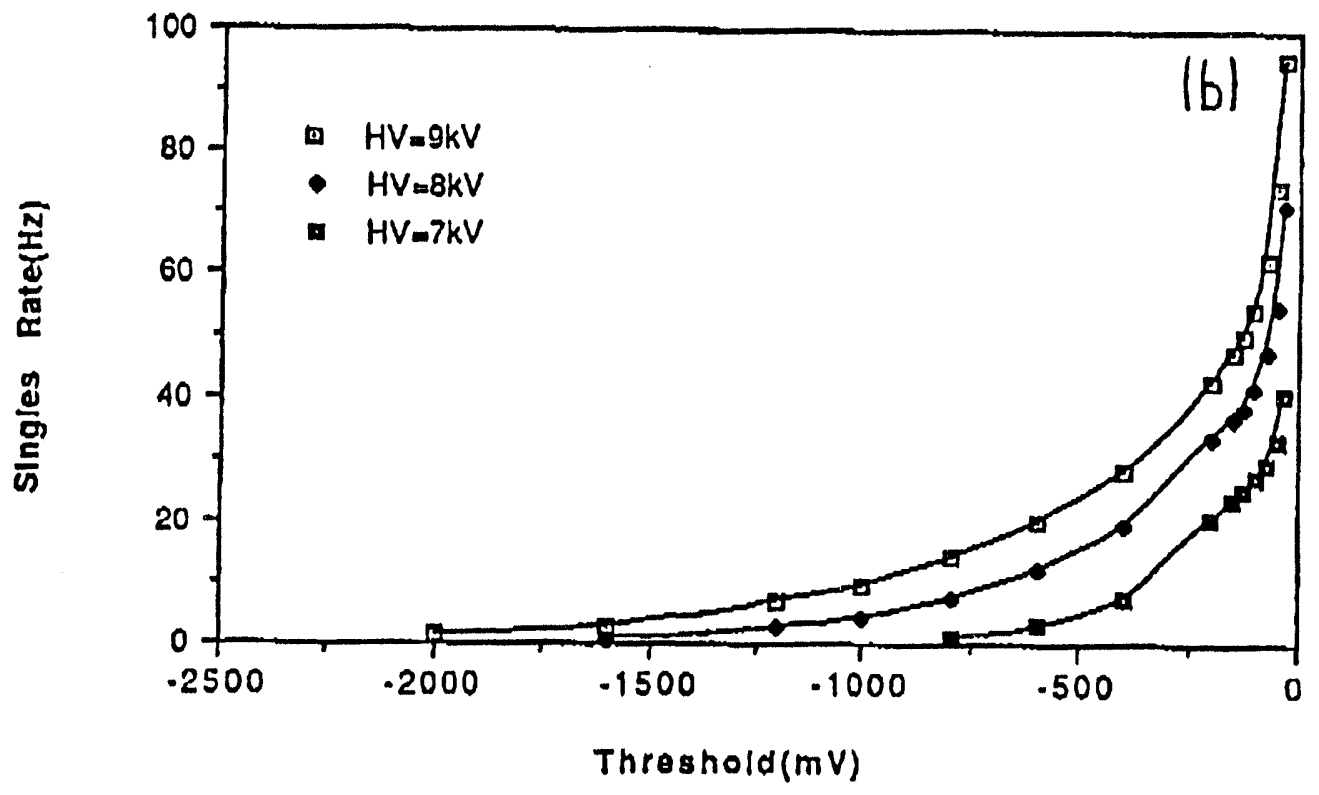

FIG.VI 

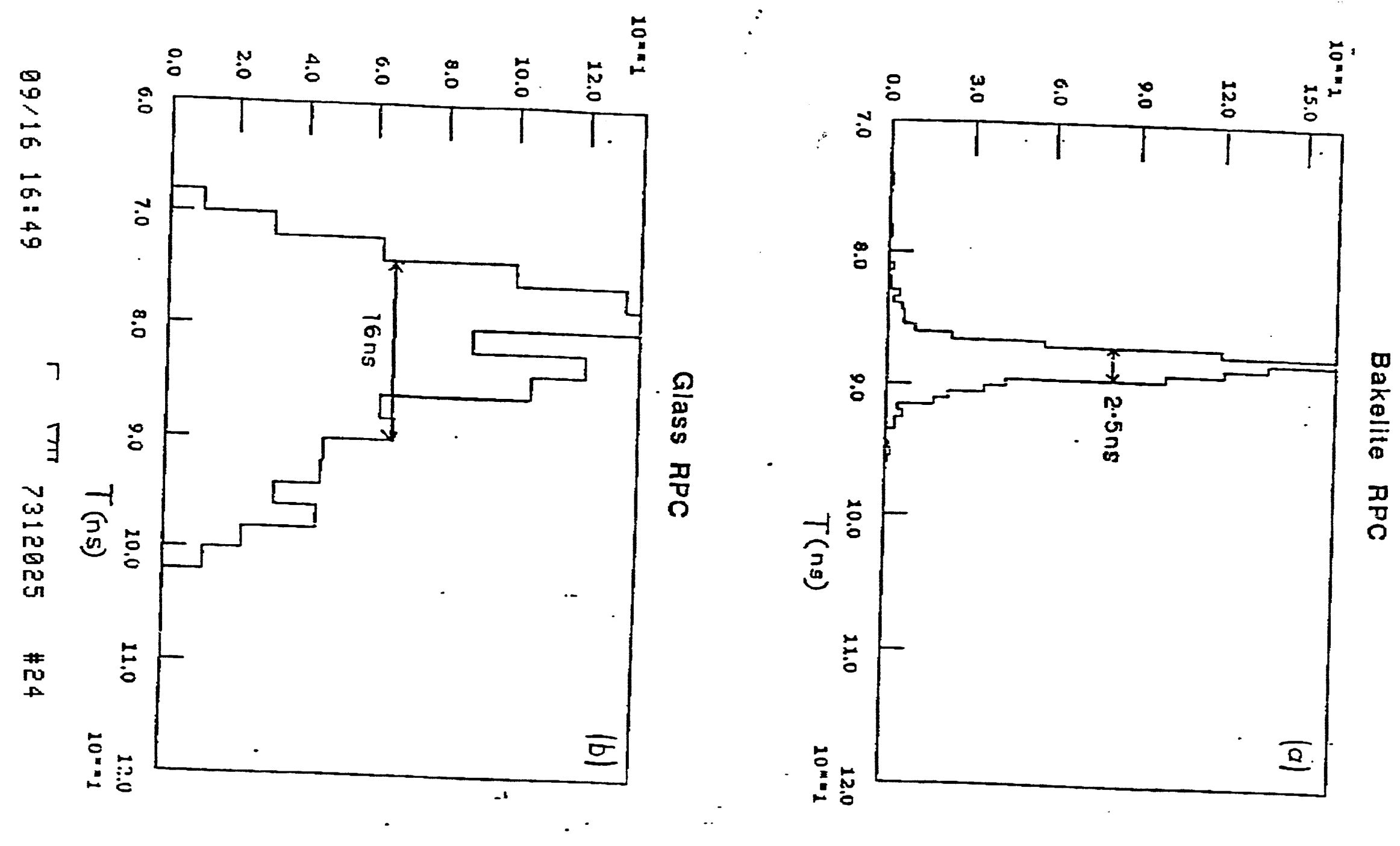

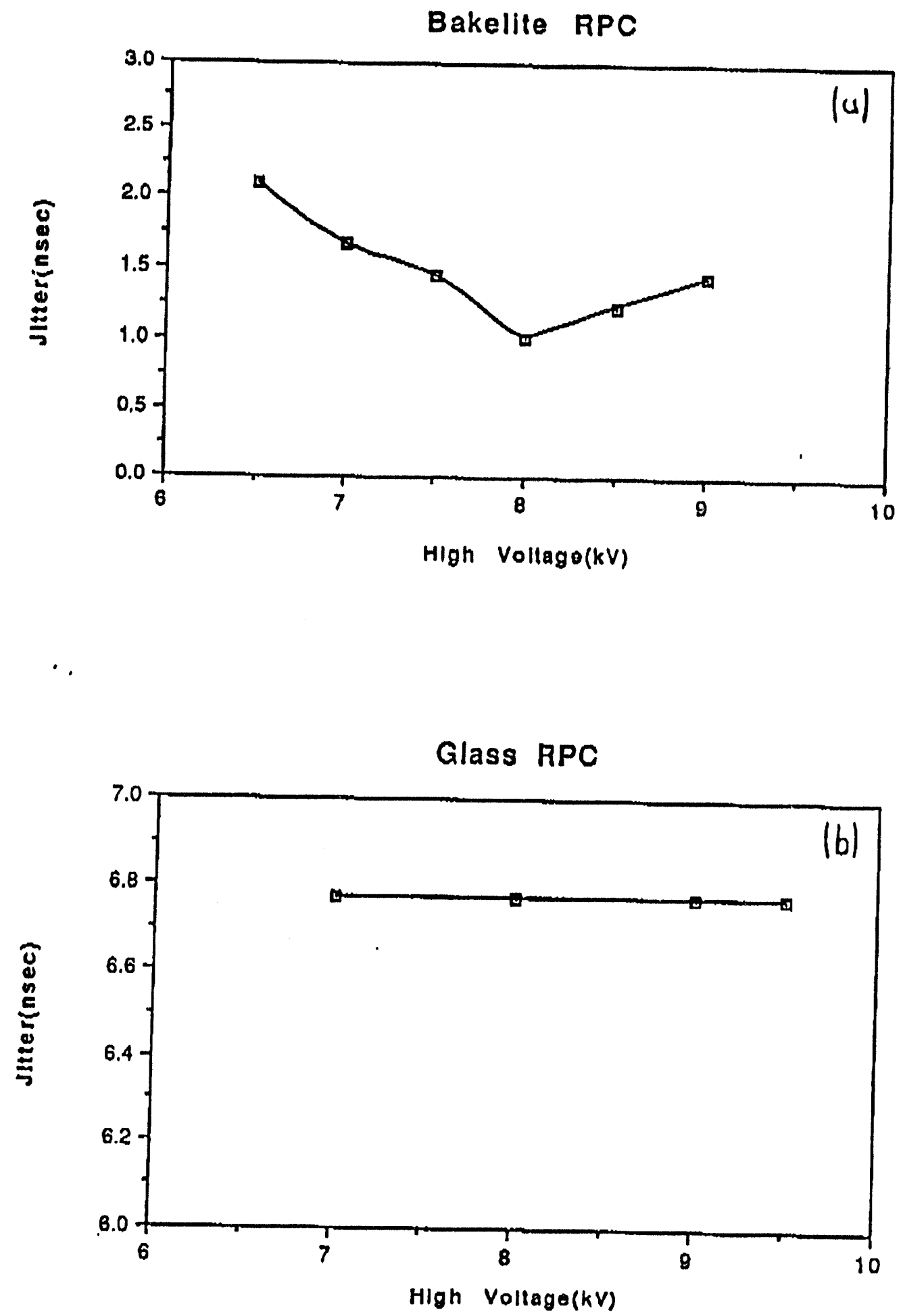

$F_{10}$. IIIII 


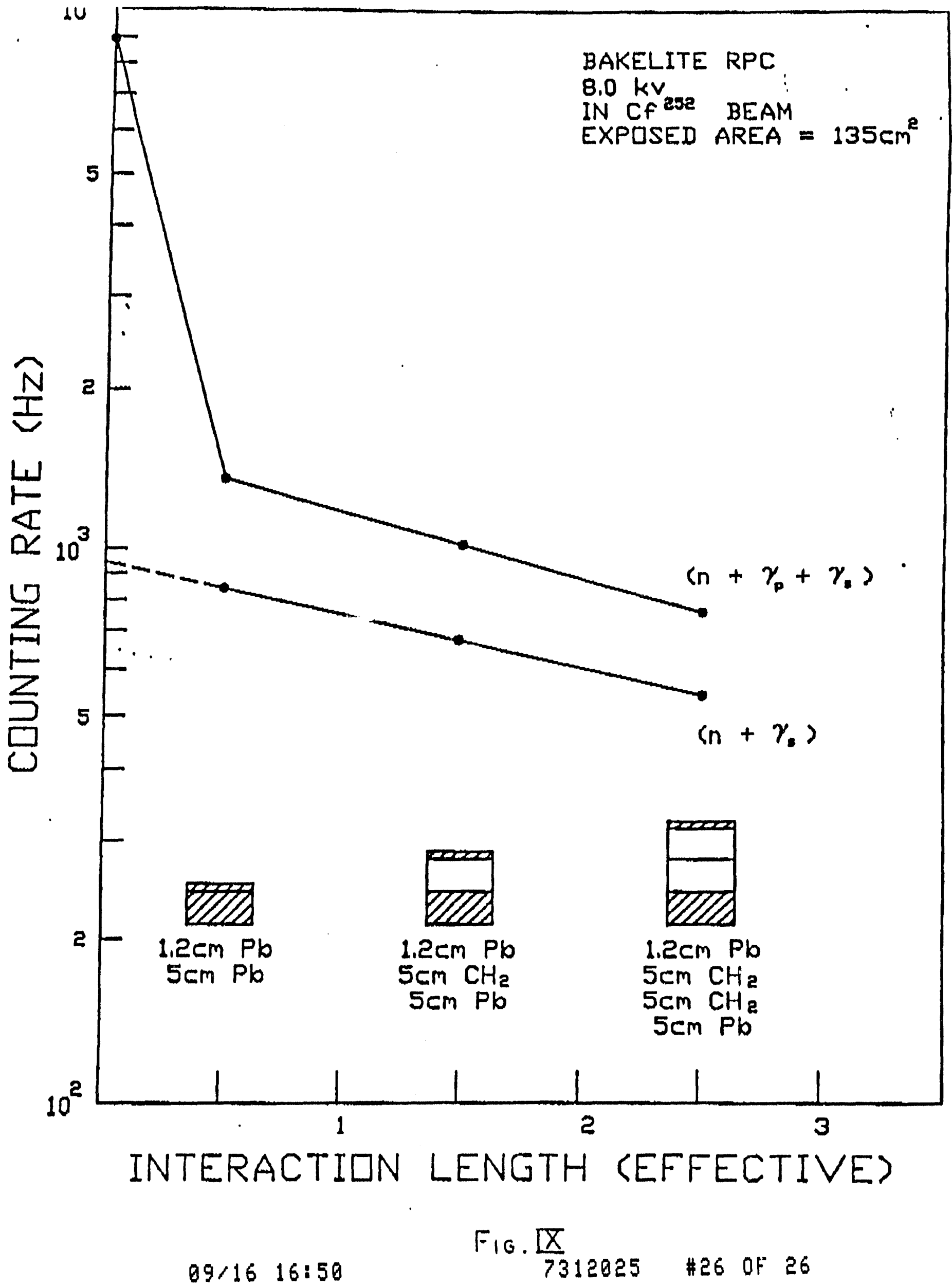




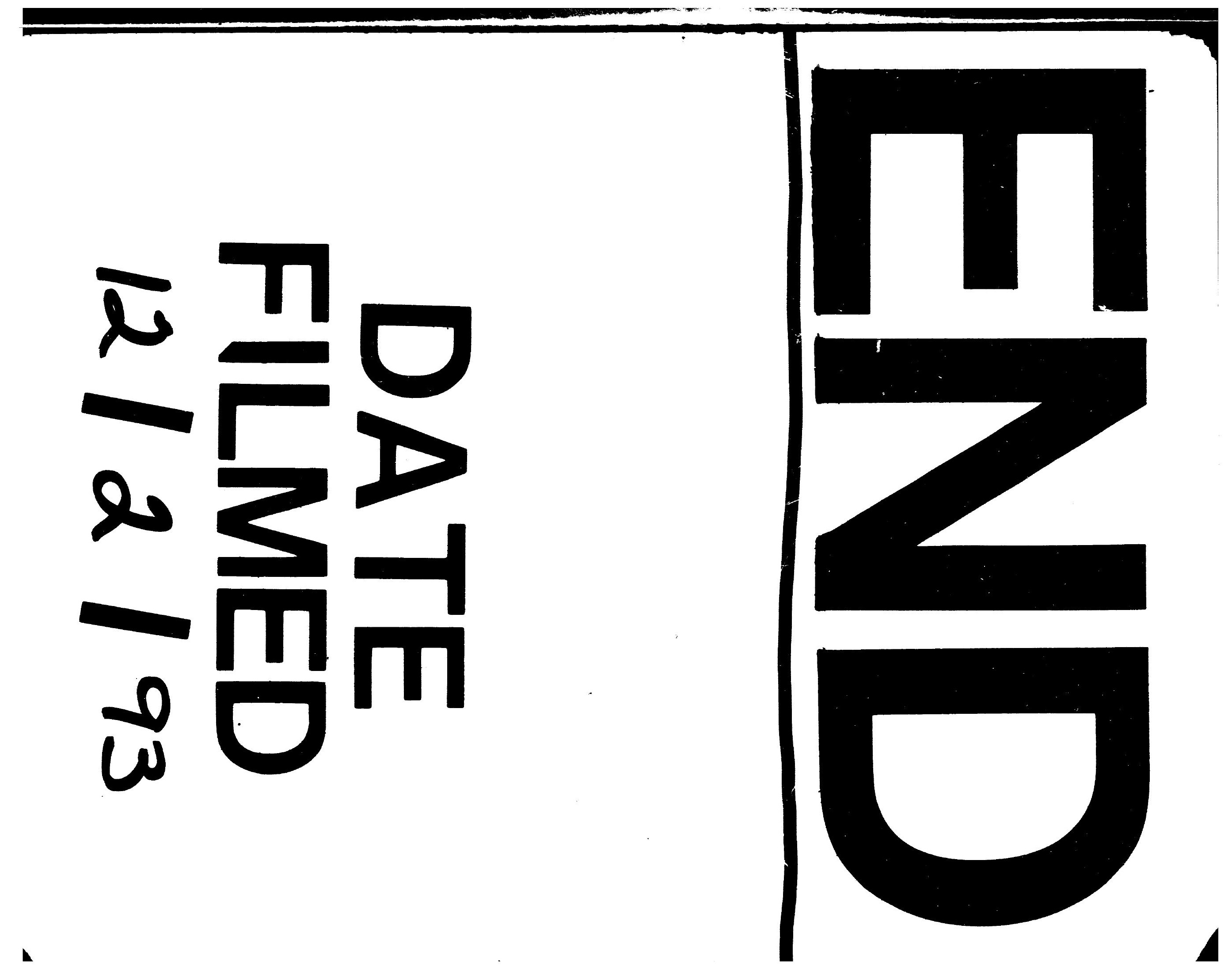


